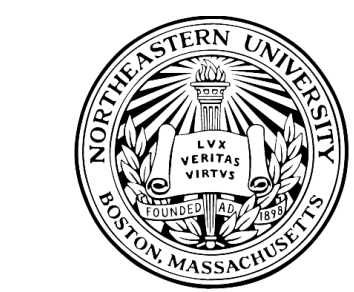

Northeastern University

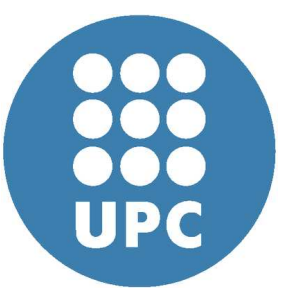

Universitat Politècnica de Catalunya

\title{
SPACE-FREQUENCY CODED OFDM FOR UNDERWATER ACOUSTIC COMMUNICATIONS
}

\author{
MASTER OF SCIENCE THESIS
}

in partial Fulfillments for the Degree of Telecommunication Engineering

\author{
at the \\ Universitat Politècnica de Catalunya
}

Author:

Eduard VALERA I ZORITA
Advisor:

Dr. Milica Stojanovid 



\section{Contents}

$\begin{array}{ll}\text { Acknowledgements } & 11\end{array}$

Resum 13

$\begin{array}{ll}\text { Resumen } & 17\end{array}$

$\begin{array}{ll}\text { Abstract } & 21\end{array}$

1 Underwater acoustic channel $\quad 23$

1.1 Attenuation and noise . . . . . . . . . . . . . . . . . 23

1.2 Multipath channel . . . . . . . . . . . . . . . . . . . 28

1.3 The Doppler effect . . . . . . . . . . . . . . . . . . . 30

2 Orthogonal Frequency Division Multiplexing 33

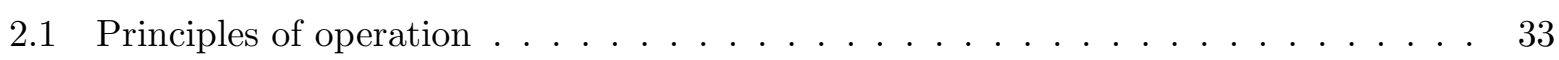

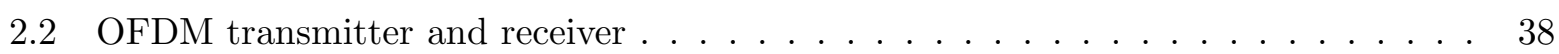

2.3 Inter-carrier interference . . . . . . . . . . . . . . . . . . . . . 41

2.4 System overview . . . . . . . . . . . . . . . . . . . . . . . . . 42

3 Multiple-input multiple-output communications 43

3.1 MIMO channel model . . . . . . . . . . . . . . . . . . . . . . . . . . . . 44

3.2 Diversity and multiplexing . . . . . . . . . . . . . . . . . . 44

3.2 .1 Diversity gain . . . . . . . . . . . . . . . . . . . . . 44

3.2 .2 Multiplexing gain . . . . . . . . . . . . . . . . . 46

3.3 Diversity-multiplexing tradeoff $\ldots \ldots \ldots \ldots \ldots \ldots$

3.4 Transmit diversity . . . . . . . . . . . . . . . . . . . . 52

3.4.1 Transmit diversity with channel knowledge . . . . . . . . . . . . 52

3.4 .2 Transmit diversity over an unknown channel . . . . . . . . . . . . 53

4 SFBC-OFDM system for acoustic channels $\quad \mathbf{6 1}$

4.1 System model . . . . . . . . . . . . . . . . . . . . . . . . . . 61

4.1 .1 Channel model . . . . . . . . . . . . . . . . . . . . . 62 
4.1 .2 The Alamouti assumption . . . . . . . . . . . . . . . . . . 63

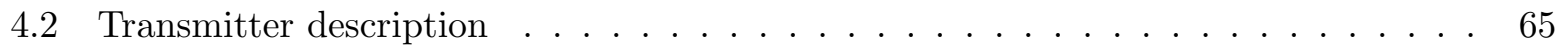

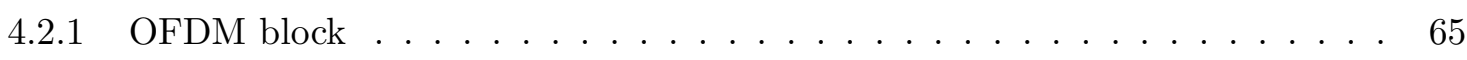

4.2 .2 Frame structure $\ldots \ldots \ldots \ldots \ldots \ldots \ldots \ldots$

4.3 Front-end processing at the receiver $\ldots \ldots \ldots \ldots \ldots \ldots$

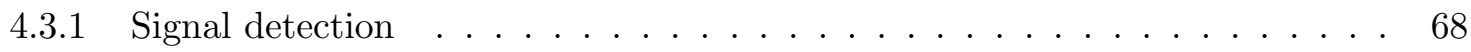

4.3 .2 Doppler compensation . . . . . . . . . . . . . . . . 69

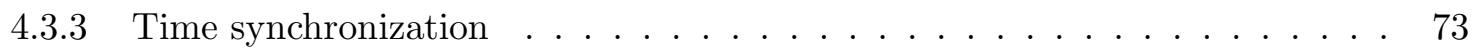

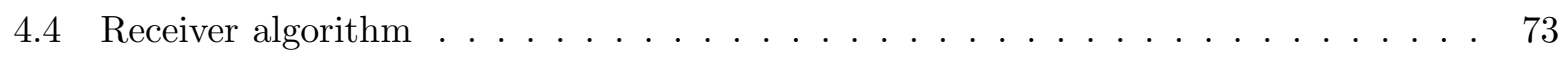

4.4 .1 One-shot channel estimation . . . . . . . . . . . . . . . 74

4.4.2 Adaptive channel estimation . . . . . . . . . . . . . 77

5 Results $\quad 83$

5.1 Experiment description $\ldots \ldots \ldots \ldots \ldots \ldots \ldots$

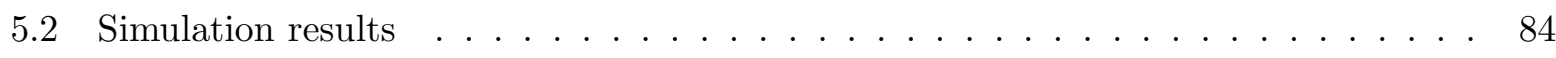

5.2 .1 System performance . . . . . . . . . . . . . . . . . . 85

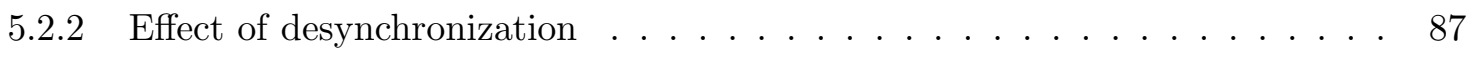

5.2 .3 Effect of increased channel variation . . . . . . . . . . . . . . . 88

5.2 .4 Effect of Doppler distortion . . . . . . . . . . . . . . . . 88

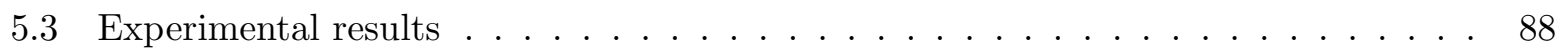

5.3 .1 System performance . . . . . . . . . . . . . . . . 89

5.3 .2 Effect of desynchronization . . . . . . . . . . . . . . . . 91

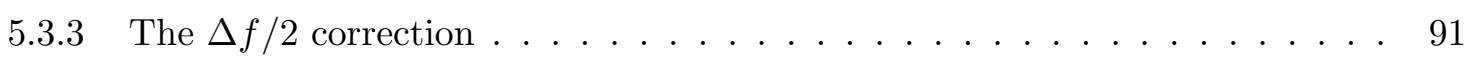

5.3.4 Comparison of sparse channel estimation methods . . . . . . . . . . . . 93

$\begin{array}{lll}6 & \text { Conclusions and future work } & 97\end{array}$

$\begin{array}{ll}\text { A Mathematical proofs } & 99\end{array}$

A.1 Trace inequalities . . . . . . . . . . . . . . . . . . . . . . . . 99 


\section{List of Figures}

1.1 Absorption coefficient $a(f)$ expressed in $\mathrm{dB} / \mathrm{km} . \ldots \ldots \ldots \ldots \ldots$

1.2 Site-specific noise p.s.d. . . . . . . . . . . . . . . . 25

1.3 Noise p.s.d with approximation. . . . . . . . . . . . . . . 25

1.4 Evaluation of $1 / A(l, f) N(f)$ for spreading factor $k=1.5$, moderate shipping activity $(s=0.5)$, no wind $(w=0 \mathrm{~m} / \mathrm{s})$ and distances $l=\{1,2,5,10,50,100\} \mathrm{km} \ldots 26$

1.5 Optimal frequency $f_{0}$ and $3 \mathrm{~dB}$ bandwidth margins as a function of distance. . . . 27

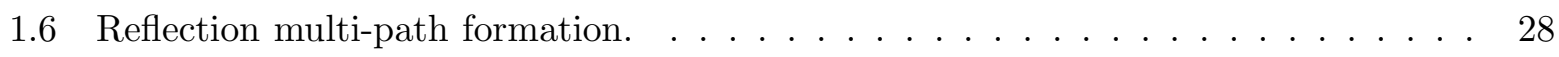

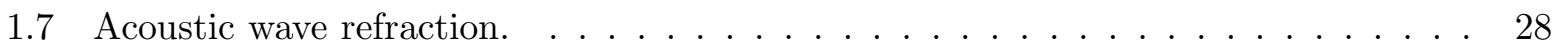

1.8 Non-uniform frequency shifting in a wideband system, caused by motion-induced Doppler distortion. . . . . . . . . . . . . . . . . . . . . . 31

2.1 OFDM signal. $K=8, B=1 \mathrm{kHz}, T_{g}=10 \mathrm{~ms} \ldots \ldots \ldots \ldots \ldots$

2.2 Spectrum of an OFDM signal. $K=8, B=1 \mathrm{kHz}, f_{0}=5 \mathrm{kHz} . \ldots \ldots$

2.3 Block diagram of an OFDM transmitter. . . . . . . . . . . . . . 38

2.4 Block diagram of an OFDM receiver. . . . . . . . . . . . . . 38

2.5 Constellation of a 16-QAM modulation scheme. . . . . . . . . . . . . . 39

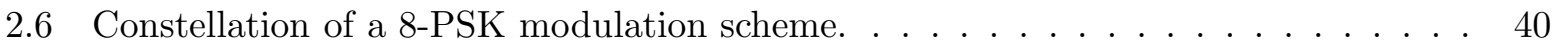

2.7 Interleaving scheme for an OFDM system. . . . . . . . . . . . . . . . . 40

2.8 Inter-carrier interference produced by Doppler shifting. . . . . . . . . . . . . . . 41

3.1 (a) Receive diversity. (b) Transmit diversity. (c) Both transmit and receive diversity. 45

3.2 MIMO channel converted into a parallel channel through SVD . . . . . . . . . . 47

3.3 Schematic of the water-filling algorithm. . . . . . . . . . . . . . 49

3.4 Tradeoff for PAM and QAM. . . . . . . . . . . . . . . 51

3.5 Tradeoff for $2 \mathrm{x} 2 \mathrm{MIMO}$ schemes. . . . . . . . . . . . . . . . . . 51

3.6 Optimal diversity-multiplexing tradeoff curve for a $M_{T} \times M_{R}$ MIMO system. . . . 52

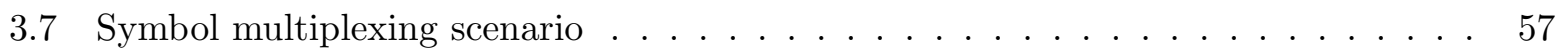

3.8 Multiplexed symbol receiver with ISI cancellation . . . . . . . . . . . 58

4.1 MSE introduced by the inaccuracy of the Alamouti assumptions. . . . . . . . . . 65

4.2 Alamouti-OFDM transmitter scheme. . . . . . . . . . . . . . 66

4.3 OFDM frame scheme. . . . . . . . . . . . . . . . . . 67 


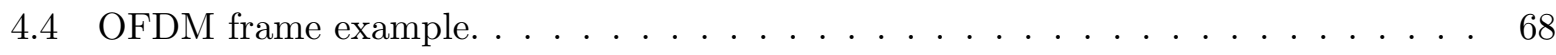

4.5 Downconverter filter response. . . . . . . . . . . . . . . . . . . . . . 69

4.6 Received frame-preamble. . . . . . . . . . . . . . . . . . . 71

4.7 Received frame-postamble. . . . . . . . . . . . . . . . . . 71

4.8 Cross correlation between sent and received preamble. . . . . . . . . . . . . . . 71

4.9 Cross correlation between sent and received postamble. . . . . . . . . . . . . 71

4.10 Cross correlation between previously correlated preamble and postamble. . . . . . 72

4.11 Detailed zoom. The cross correlation presents a maximum at sample 27 . . . . . . 72

4.12 Doppler shift compensation using data interpolation. . . . . . . . . . . . . . . 73

4.13 Adaptive thresholding example . . . . . . . . . . . . . . . . 76

4.14 Block diagram of the adaptive receiver algorithm. . . . . . . . . . . . . 78

4.15 Scatter plot of real data using a receiver without (left) and with (right) phase tracking. 79

4.16 Measured Doppler evolution in experimental data for transmitters 1 and 2. Each line represents the residual Doppler observed in one receiving element. . . . . . . . 80

4.17 Channel gain variation during one OFDM frame, obtained from experimental data. 81

4.18 BER vs adaptation factor $\lambda \ldots \ldots \ldots \ldots \ldots \ldots \ldots$. . . . . . . . . . . . . . . . .

4.19 MSE vs adaptation factor $\lambda \ldots \ldots \ldots \ldots \ldots \ldots \ldots$. . . . . . . . . . . . . .

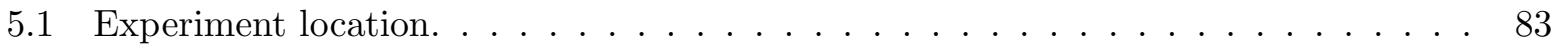

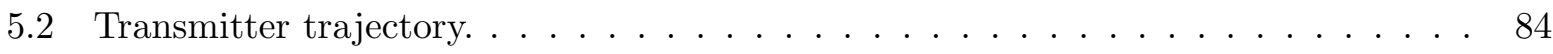

5.3 Experiment geometry. . . . . . . . . . . . . . . . . . . . . 84

5.4 Snapshots of channel response observed between the two Alamouti transmitters

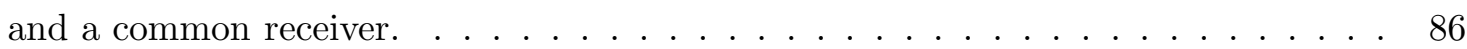

5.5 Simulation: BER vs. number of carriers. $\mathrm{SNR}=15 \mathrm{~dB}, M_{R}=2$ receiving elements, channel Doppler spread $B_{d}=1 \mathrm{~Hz}$. Label X indicates full channel inversion (4.3).

5.6 Performance comparison between SIMO, STBC and SFBC with different channel estimation algorithms: least-squares with adaptive thresholding (AT) and orthogonal matching pursuit (OMP). $K=256, M_{R}=2$ receivers. . . . . . . . . . .

5.7 Performance sensitivity to synchronization mismatch between transmitters: MSE vs. delay difference $\Delta \tau_{0}(0) . K=256$ carriers, $M_{R}=6$ receiving elements. . . . . 88

5.8 Performance comparison between SIMO, STBC and SFBC for different channel variation rates. $\mathrm{SNR}=20 \mathrm{~dB}, K=256, M_{R}=2$ receivers. $\ldots \ldots \ldots \ldots$

5.9 Performance comparison between SIMO, STBC and SFBC for different residual relative velocities. $\mathrm{SNR}=15 \mathrm{~dB}, K=256, M_{R}=2$ receivers, $B_{d}=1 \mathrm{~Hz}$. . . . .

5.10 Experiment: BER (uncoded) vs. the number of carriers. $M_{R}=12$ receiving elements. Each point represents an average over all carriers and frames. . . . . . .

5.11 MACE experiment, day 5: MSE evolution in time. $K=256, M_{R}=12$ receiving elements. 
5.12 Performance sensitivity to synchronization mismatch between transmitters: MSE vs. delay difference $\Delta \tau_{0}(0)$. MACE'10 data with $K=256$ carriers, $M_{R}=12$ receiving elements. . . . . . . . . . . . . . . . . . . . 92

5.13 System performance with and without the $\Delta f / 2$ correction. Results are shown for a single MACE'10 frame. $M_{R}=12$ receiving elements. . . . . . . . . . . . . . 92

5.14 Comparison between adaptive-threshold (20 steps) and fixed-threshold methods; single MACE'10 frame. $M_{R}=12$ receiving elements. . . . . . . . . . . . . 93

5.15 Adaptive threshold values for different tx/rx pairs during transmission of one MACE'10 frame. $M_{R}=12$ receiving elements, $K=256$ carriers. . . . . . . . . . 94

5.16 Comparison between adaptive-threshold (20 steps), OMP and Algorithm [21]; single MACE'10 frame. $M_{R}=12$ receiving elements. . . . . . . . . . . . . . . 94

5.17 Example of channel responses (magnitude) estimated by the OMP and the LS-AT

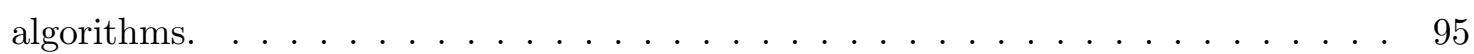





\section{List of Tables}

4.1 OFDM modulation parameters . . . . . . . . . . . . . . . . . 66

4.2 OFDM frame parameters . . . . . . . . . . . . . . . 67

4.3 Measured Doppler shift (samples) . . . . . . . . . . . . . . . 72

5.1 MACE Experiment signal parameters . . . . . . . . . . . . . . . 85

5.2 Computational complexity of sparse channel estimation algorithms for an OFDM

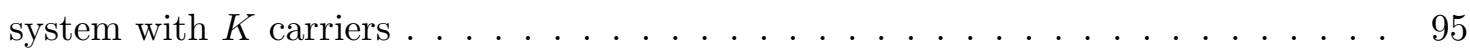





\section{Acknowledgements}

First of all, I would like to thank all the professors who dedicate their lives to teaching our future generations and helping them to become the best that they can be. Also, I feel indebted with the society, which has made possible my education in such excellent public schools and universities. Among all the professors I had in my life, I especially remember those who made me love the science as much as they do. Thanks to Josep Vidal for helping me discover the interesting area of signal processing and inviting me to join his research group, that experience not only served as an excellent training of advanced topics, but also has become my first approach to the world of scientific research.

Thanks to Milica for making possible such enriching experience and for the invaluable things I have learnt during this year at Northeastern University and at MIT. Her friendly relation with her students made this stay much more pleasant to me, and her incredible analytical mind kept me motivated to continue learning and improving every day. My colleagues at Northeastern have been a great support to carry out this project, especially Fatemeh and Yashar. We had exciting conversations, lots of laughs, as well as ping-pong games, and they gave me all the advice I needed both in my academic and non-academic issues. Their presence definitely made my days more enjoyable. I would also like to thank Fatemeh and Shana for their interesting reviews of this thesis.

My most sincere thanks to my family and friends, who believe in my capabilities much more than I do. All this wouldn't have been possible without their unconditional support since I started my degree. This thesis is also for Carlota, for making everything so easy with her enthusiasm to see me growing and fulfilling my dreams. Last, and most important, thanks to my mother.

Eduard 



\section{Resum}

En els últims anys el volum d'investigació en el camp de les comunicacions subaquàtiques ha augmentat considerablement. L'increment en el nombre d'aplicacions científiques, com per exemple el control de la pol-lució i les espècies marines autòctones, la monitorització de moviments sísmics del sòl marí o l'exploració subaquàtica del gel de l'Àrtic, entre d'altres, ha motivat la recerca més enllà del camp militar. Aquestes aplicacions requereixen, en la majoria de casos, l'ús de xarxes de sensors o de vehicles subaquàtics no tripulats, que habitualment es comuniquen utilitzant xarxes sense fils a fi d'evitar les restriccions de mobilitat i la complexitat que suposa la instal-lació de cablejat submarí.

La forta atenuació que pateixen les ones electromagnètiques en el medi aquàtic fa que el seu ús es restringeixi només a enllaços d'alta capacitat però curt abast - de l'ordre de centímetres. Les comunicacions de propòsit general s'estableixen mitjançant ones acústiques. En aquests casos, la velocitat de propagació d'ona és cinc ordres de magnitud inferior i juga un paper molt important, degut a que els retards de propagació en enllaços de pocs quilòmetres són de l'ordre de segons. A més, el moviment relatiu entre transmissor i receptor genera una severa distorsió per efecte Doppler que requereix processament de senyal addicional en l'etapa de recepció. El canal subaquàtic també es caracteritza per la propagació multicamí, provocada per les reflexions de l'ona amb la superfície i el fons marí, així com la refracció produïda pel medi.

Les dificultats esmentades compliquen molt el disseny d'un sistema que sigui alhora fiable i amb una capacitat de transmissió raonable. L'objectiu d'aquest projecte és el disseny d'un sistema que transmet codis espai-freqüència, amb la finalitat d'obtenir diversitat en transmissió i millorar de forma notable la qualitat de l'enllaç de comunicacions. La motivació per a investigar els codis espai-freqüència recau en el fet que recentment s'han obtingut resultats esperançadors amb l'ús de codis espai-temps. No obstant això, la variació del canal limita molt el rendiment dels codis espai-temps, ja que un sol codi s'estén a més d'un bloc temporal. L'objectiu és evitar aquest efecte canviant la dimensió temporal per la freqüencial però mantenint l'estructura i els beneficis del codi original. D'aquesta manera, les diferents parts del codi es poden multiplexar simultàniament en diferents freqüències i la transmissió completa s'assoleix en un sol bloc, i.e. un sol ús del canal.

En aquest projecte s'utilitza com a modulació la multiplexació en divisió de freqüència ortogonal (OFDM per les seves sigles en anglès), principalment perquè ofereix un mètode molt senzill per equalitzar els canals selectius. La modulació OFDM també ofereix una versatilitat molt interessant pel que fa a la reconfiguració de l'ample de banda i l'espaiat de portadores. A més, 
l'ús combinat de l'OFDM i els sistemes multi-antena (MIMO) simplifica molt el processament de senyal, ja que cada portadora pot ser tractada com un canal no selectiu.

Els sistemes MIMO s'utilitzen en canals acústics subaquàtics tant per incrementar la velocitat de transmissió [1, 2] (multiplexat en espai), com per millorar el rendiment dels sistemes aprofitant la diversitat espacial [3]. En el nostre cas, el sistema MIMO s'utilitza per aconseguir diversitat en transmissió a partir de l'ús del codi d'Alamouti [4] emprat en domini freqüencial. Els codis de bloc en espai-freqüència (SFBC) són més adequats per a la modulació OFDM que els codis espai-temps (STBC). Això és degut a que els blocs OFDM solen tenir una durada considerable que pot comprometre la condició de coherència, la qual requereix que el canal es mantingui constant durant tots els blocs temporals que ocupa el codi [3]. Pel que fa als codis SFBC, aquesta coherència del canal s'ha de respectar per dues freqüències portadores consecutives. Afortunadament, aquest requisit coincideix amb la condició de disseny de la modulació OFDM, en la qual les portadores adjacents han de ser prou properes com per a que el canal es pugui considerar constant entre l'una i l'altra. A la literatura hi trobem resultats recents per comunicacions radioelèctriques que proven que hi ha situacions en les quals els codis SFBC tenen millor rendiment que els STBC $[5,6]$.

Existeixen principalment dos tipus de receptors MIMO-OFDM en comunicacions subaquàtiques: no adaptatius, en els quals cada bloc rebut és processat independentment dels altres i el canal s'estima mitjançant símbols pilot [1], i adaptatius, els quals aprofiten la coherència del canal entre blocs consecutius per fer prediccions de la futura funció de transferència i així reduir el nombre de símbols pilot [2]. En tots dos casos cal una primera etapa de sincronització i correcció de la distorsió Doppler, la qual es fa de la mateixa manera en tots els receptors si aquests es troben co-localitzats [7]. En cas contrari, cal un algorisme de compensació paral-lela [8].

El disseny de l'algorisme de recepció per codis SFBC està basat en el receptor MIMO-OFDM adaptatiu. Més concretament, el receptor separa el canal en dos factors: el guany del canal, que té una variació lenta, i una fase que varia ràpidament. L'essència del receptor és que pot estimar aquests dos paràmetres a diferents velocitats. Per a estimar el canal s'utilitza tant el mètode OMP [9] com un nou algorisme que es presenta en aquest treball, el qual està basat en una estimació per mínims quadrats amb un llindar de truncament adaptatiu. Aquest últim, anomenat least squares with adaptive thresholding (LS-AT), pren la resposta impulsional del canal i la trunca a partir d'un llindar amb la finalitat de mantenir només els coeficients rellevants. En aquest cas, a diferència dels mètodes habituals de truncament amb llindar fix [2], el llindar es determina de forma adaptativa per oferir la millor separació possible entre coeficients significants i soroll. L'algorisme LS-AT ofereix un rendiment molt semblant a l'OMP amb una càrrega computacional molt més baixa.

Els beneficis de l'ús de codis espai-freqüència s'avaluen en aquest projecte mitjançant simulació matemàtica i transmissions experimentals realitzades a l'oceà Atlàntic. El guany observat es troba estretament lligat amb la condició de coherència en el domini freqüencial, de manera que el guany és major quan es disminueix l'espaiat entre portadores. Paral-lelament, per espaiats freqüencials menors, la longitud del bloc OFDM augmenta i es comença a observar l'aparició d'interferència 
inter-portadora (ICI), que degrada notablement el rendiment. En els resultats s'observa que l'efecte d'aquest compromís entre coherència temporal i freqüencial, porta a l'existència d'un nombre òptim de portadores. 



\section{Resumen}

En los últimos años, el volumen de investigación en el campo de las comunicaciones subacuáticas ha aumentado de forma considerable. La nueva variedad de aplicaciones científicas como, por ejemplo, el control de la polución y las especies marinas autóctonas, la monitorización de movimientos sísmicos del suelo marino o la exploración subacuática del hielo del Ártico, entre otras, han motivado su investigación más allá de las aplicaciones militares. Estas aplicaciones requieren, en la mayoría de casos, el uso de redes de sensores o de vehículos subacuáticos no tripulados que habitualmente se comunican mediante redes inalámbricas, a fin de evitar las restricciones de movilidad y la complejidad que supone la instalación de cableado submarino.

La fuerte atenuación que sufren las ondas electromagnéticas en el medio acuático restringe su uso exclusivamente a enlaces de alta capacidad pero corto alcance - del orden de centímetros. Las comunicaciones de propósito general se establecen mediante ondas acústicas. En estos casos, la velocidad de propagación de onda es cinco órdenes de magnitud inferior y juega un rol muy importante, debido a que los retrasos de propagación en enlaces de pocos kilómetros son del orden del segundo. Además, el movimiento relativo entre transmisor y receptor genera una severa distorsión por efecto Doppler que requiere procesado de señal adicional en el receptor. El canal subacuático también se caracteriza por la propagación multicamino provocada por refracción producida por el medio y por las reflexiones de la onda con la superficie y el fondo marino.

Las dificultades mencionadas complican el diseño de un sistema que sea a la vez fiable y tenga una capacidad de transmisión razonable. El objetivo de este proyecto es diseñar un sistema que transmita códigos espacio-frecuencia, con la finalidad de obtener diversidad en transmisión y mejorar de forma notable la calidad del enlace de comunicaciones. La motivación para investigar los códigos espacio-frecuencia se basa en el hecho que recientemente se han obtenido resultados esperanzadores con el uso de códigos espacio-tiempo. No obstante, la variación de canal limita de forma considerable el rendimiento de los códigos espacio-tiempo, ya que el código se extiende a más de un bloque temporal. El objetivo es evitar este efecto cambiando la dimensión temporal por frecuencia aún manteniendo la estructura y beneficios del código original. Así, las diferentes partes del código son multiplexadas de forma simultánea en diferentes frecuencias y la transmisión completa del código se consigue con un solo uso de canal.

En este proyecto se utiliza como modulación el multiplexado en división de frecuencia ortogonal (OFDM), principalmente porqué ofrece un método muy sencillo para ecualizar canales selectivos. La modulación OFDM también ofrece una versatilidad muy interesante para reconfigurar el ancho 
de banda y el espaciado entre frecuencias portadoras. Además, el uso combinado de OFDM y los sistemas multiple-input multiple-output (MIMO) simplifica notablemente el procesado de señal, ya que cada portadora puede ser tratada como un canal no selectivo.

Los sistemas MIMO se usan en canales acústicos subacuáticos tanto para incrementar la velocidad de transmisión $[1,2]$ como para mejorar el rendimiento de los sistemas aprovechando la diversidad espacial [3]. En nuestro caso, se usa un sistema MIMO con el objetivo de obtener diversidad en transmisión a partir del uso de códigos Alamouti [4] transformados al dominio frecuencial. Los códigos bloque espacio-frecuencia (SFBC) son más adecuados para la modulación OFDM que los códigos espacio-tiempo (STBC). Esto se debe a que los bloques OFDM suelen tener una duración considerable que puede comprometer la condición de coherencia, la cual exige un canal constante durante todos los bloques que conforman el código [3]. Cuando se usan códigos SFBC, la coherencia de canal debe ser respetada de la misma manera pero durante dos portadoras adyacentes. Afortunadamente este requisito coincide con la condición de diseño de la modulación OFDM, en la cual las portadoras adyacentes deben ser suficientemente cercanas como para que el canal pueda considerarse constante. En la literatura encontramos resultados recientes para comunicaciones radioeléctricas que demuestran situaciones en las que los códigos SFBC tienen un rendimiento superior a los códigos $\mathrm{STBC}[5,6]$.

Existen principalmente dos tipos de receptores MIMO-OFDM para comunicaciones subacuáticas: no adaptativos, en los que cada bloque se procesa independientemente de los demás y el canal se estima usando símbolos piloto [1], y adaptativos, en los que se aprovecha la coherencia del canal entre bloques consecutivos para hacer predicciones del canal futuro y reducir el número total de símbolos piloto [2]. En ambos casos se requiere una etapa de sincronización y corrección de la distorsión Doppler, que se aplica de la misma manera en todos los receptores si estos se encuentran co-localizados [7]. En caso contrario la compensación se realiza con un algoritmo de compensación paralela [8].

El diseño del algoritmo de recepción para códigos SFBC está basado en el receptor MIMOOFDM adaptativo. El receptor separa el canal en dos factores: la amplitud del canal, que tiene una variación lenta, y una fase de variación rápida. La esencia del receptor es la posibilidad de estimar estos parámetros de forma paralela a diferentes velocidades. Para la estimación de canal se utilizan alternativamente el método OMP [9], o bien un nuevo algoritmo presentado en este proyecto basado en estimación por mínimos cuadrados con un umbral de truncamiento adaptativo. El nuevo método, que se denomina least squares with adaptive thresholding (LS-AT), trunca la respuesta impulsional del canal con la finalidad de mantener solamente los coeficientes relevantes. En este caso, a diferencia de los métodos habituales de truncamiento con umbral fijo [2], el umbral se determina de forma adaptativa para ofrecer la mejor separación posible entre canal y ruido. El algoritmo LS-AT ofrece un rendimiento muy cercano al del algoritmo OMP pero requiere una carga computacional considerablemente menor.

Los beneficios del uso de códigos espacio-frecuencia se evalúan mediante simulación matemática y mediante transmisiones experimentales realizadas en el Océano Atlántico. Los beneficios obtenidos 
están estrechamente ligados a la condición de coherencia en el dominio frecuencial, de modo que la ganancia es mayor cuando se disminuye el espaciado entre portadoras. A la vez que se reduce el espaciado frecuencial, la longitud temporal del bloque OFDM aumenta y se observa la aparición de interferencia inter-portadora (ICI), la cual degrada notablemente el rendimiento. En los resultados se observa que el efecto de este compromiso lleva a la existencia de un número óptimo de portadoras. 



\section{Abstract}

In recent years, the growing number of oceanographic applications that rely on underwater communications has motivated extensive research in the field. These scientific projects usually require data acquisition from sensor networks or the use of unmanned underwater vehicles. One method to establish communication with such underwater systems is through the use of wired links. However, cables are hard to install or repair at certain depths, and can dramatically limit the mobility of both communication ends. Underwater wireless communications do not have such constraints and therefore present a much more attractive approach for underwater data transfer.

Electromagnetic waves typically provide higher throughputs than any other wireless communication method. However, they suffer from tremendous attenuation in water mediums. Consequently, underwater radio communications are only applicable to very short range high-speed links. For general purpose communications, acoustic waves are the preferred method. The fact that the wave propagation speed is five orders of magnitude smaller causes serious issues, such as long end-to-end delays and extreme Doppler distortion produced by the relative motion between transmitter and receiver. The underwater channel also suffers from multipath propagation produced by wave refraction, as well as reflections from the surface and the sea bed.

The aforementioned issues complicate the design of an underwater acoustic system, which is able to offer both reliability and a reasonable communication speed at the same time. The aim of this work is to increase the robustness of the state-of-the-art underwater communication schemes. We achieve this goal using multiple transmitting and receiving elements, where each transmitterreceiver combination counts as an additional communication channel. The increased number of parallel channels drastically reduces the the error probability of the link, i.e. the probability that all channels are experiencing simultaneous fading.

Orthogonal frequency division multiplexing (OFDM) is considered for frequency-selective underwater acoustic (UWA) channels as it offers low complexity of fast Fourier transform-based (FFT) signal processing, and ease of reconfiguration for use with different bandwidths. In addition, by virtue of having a narrowband signal on each carrier, OFDM is easily conducive to multi-input multi-output (MIMO) system configurations.

MIMO systems have been considered for UWA channels both for increasing the system throughput via spatial multiplexing $[1,2]$ and for improving the systems performance via spatial diversity [3]. The focus of our present work is on transmit diversity, which we pursue through the use of Alamouti coding applied across the carriers of an OFDM signal. Space-frequency block coding 
(SFBC) is chosen over traditional space-time block coding (STBC) as better suited for use with acoustic OFDM signals. Namely, while the Alamouti coherence assumption [4] may be challenged between two adjacent OFDM blocks on a time-varying acoustic channel [3], it is expected to hold between two adjacent OFDM carriers: frequency coherence assumption coincides with the basic OFDM design principle which calls for carriers to be spaced closely enough that the channel transfer function can be considered flat over each sub-band. Previous studies in radio communications have also revealed situations in which SFBC outperforms STBC $[5,6]$.

Two types of approaches have been considered for MIMO OFDM acoustic systems: nonadaptive, where each block is processed independently using pilot-assisted channel estimation [1], and adaptive, where coherence between adjacent blocks is exploited to enable decision-directed operation and reduce the pilot overhead [2]. Both approaches require front-end synchronization for initial Doppler compensation through signal resampling [7]. Front-end processing remains unchanged for multiple transmitters if they are co-located and experience the same gross Doppler effect. Otherwise, multiple resampling branches may be needed to compensate for transmitterspecific Doppler shifting [8].

Leveraging on the adaptive MIMO-OFDM design, we develop a receiver algorithm for the SFBC scenario. Specifically, we decouple the channel distortion into a slowly-varying gain and a faster-varying phase, which enables us to track these parameters at different speeds. For estimating the channel, we use either the orthogonal matching pursuit (OMP) algorithm [9] or a newly developed algorithm based on least squares with adaptive thresholding (LS-AT). This algorithm computes the full-size LS solution to the impulse response (IR) domain channel representation, then truncates it to keep only the significant IR coefficients. However, unlike the typical truncated LS solutions which use a fixed truncation threshold [2], the threshold is determined adaptively so as to provide a proper level of sparseness. LS-AT is found to perform close to OMP, at a lower computational cost. Once an initial channel estimate is formed, its tracking continues via time-smoothing. Simultaneously, an estimate of the residual Doppler scale is made for each of the two transmitters, and this estimate is used to predict and update the carrier phases in each new OFDM block.

The advantages of Alamouti SFBC are contingent upon frequency coherence, which increases as more carriers are packed within a given bandwidth (the bandwidth efficiency simultaneously increases). However, there is a fine line after which inter-carrier interference (ICI) will be generated, and this line should not be crossed if simplicity of Alamouti detection is to be maintained. We assess this trade-off through simulation and experimental data processing, showing the existence of an optimal number of carriers and an accompanying transmit diversity gain. 


\section{Chapter 1}

\section{Underwater acoustic channel}

Electromagnetic propagation has shown to be an infeasible method for wireless communication in underwater environments. Large antennas and high transmission power are required for transmission over short distance (usually less than a meter). Despite suffering from high frequency attenuation, the acoustic channel offers some attractive characteristics that allow underwater communication over several kilometers. Underwater acoustic systems can only operate in the frequency band of tens of kilohertz and, consequently, they are inherently wideband $\left(B \sim f_{c}\right)$. The very low propagation speed of sound $(1500 \mathrm{~m} / \mathrm{s})$ causes high end-to-end latency and extreme Doppler distortion, which demands additional complex signal pre-processing that is not required in terrestrial radiocommunications. Furthermore, due to sound reflection and refraction phenomena, propagation occurs over multiple paths that spread tens or even hundreds of milliseconds and results in a frequency selective signal distortion. These issues pose the underwater acoustic (UWA) channel as one of the most challenging communication media.

\subsection{Attenuation and noise}

One of the main characteristics of acoustic channels is the fact that path loss strongly depends on the signal frequency due to absorption, i.e. transfer of acoustic energy into heat. In addition to the absorption loss, the signal experiences a spreading loss which increases with the distance. The attenuation is more pronounced at very low and high frequencies. Consequently, the acoustic channel acts as a band-pass filter whose passband region depends on the distance of the link. The passband region usually ranges from one to a few tens of $\mathrm{kHz}$ for distances on the order of kilometers [10]. The attenuation experienced by a signal transmitted at a frequency $f$ through a distance $l$ is given by

$$
A(l, f)=l^{k} a(f)^{l}
$$

or equivalently in $\mathrm{dB}$

$$
10 \log (A(l, f))=k \cdot 10 \log (l)+l \cdot 10 \log (a(f))
$$


where $k$ characterizes the spreading loss, with usual values between 1 and 2 . The absorption coefficient $a(f)$ is an increasing function of frequency, which can be obtained using the empirical Thorp's formula

$$
a(f[\mathrm{kHz}])=0.003+\frac{0.11 f^{2}}{1+f^{2}}+\frac{44 f^{2}}{4100+f^{2}}+2.75 \cdot 10^{-4} f^{2} \quad[\mathrm{~dB} / \mathrm{km}]
$$

The absorption coefficient, shown in Fig.1.1, increases rapidly with frequency. At $100 \mathrm{kHz}$ the attenuation due to absorption is already on the order of $35 \mathrm{~dB} / \mathrm{km}$. Thus transmission on frequencies over hundred of $\mathrm{kHz}$ is infeasible.

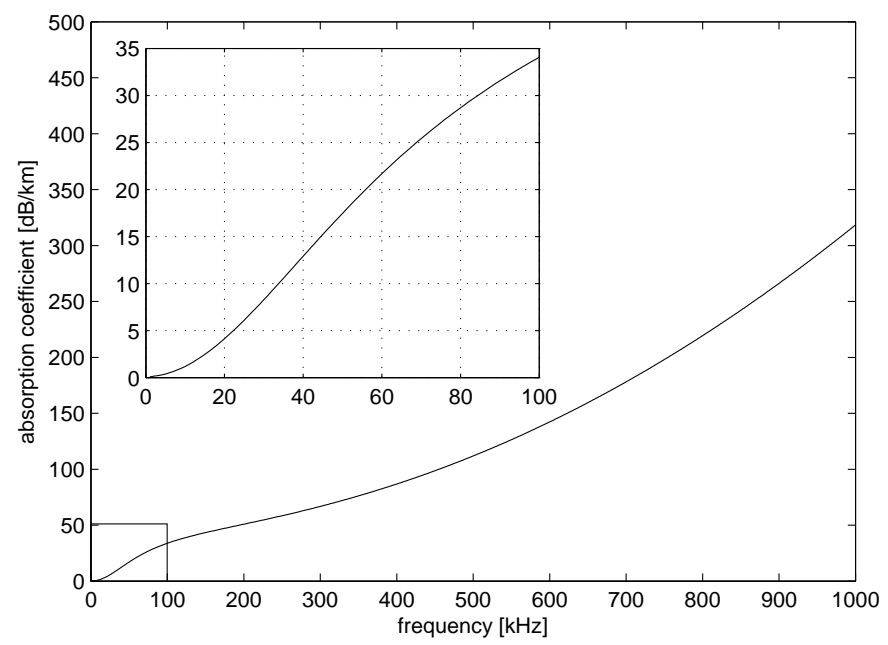

Figure 1.1: Absorption coefficient $a(f)$ expressed in $\mathrm{dB} / \mathrm{km}$.

The noise in an underwater acoustic channel stems from many different sources. The two main noise sources can be classified as: ambient noise and site-specific noise. As its name indicates, the latter contains effects that may vary depending on the place of measure, including the thermal noise and the effects of turbulence, shipping, and waves. In general, the site-specific noise can be modeled as non-white Gaussian. The noise power spectral density (p.s.d.) profiles in $\mathrm{dB}$ relative to $\mu \mathrm{Pa}$ ( $\mathrm{dB}$ re $\mu \mathrm{Pa}$ ) are empirically described by the following expressions for $f$ in $\mathrm{kHz}$ :

- Thermal noise

$$
10 \log \left(N_{t h}(f)\right)=-15+20 \log f
$$

Thermal noise does not have any influence in low frequency regions, but becomes the major noise contribution for $f>100 \mathrm{kHz}$.

- Turbulence noise

$$
10 \log \left(N_{t}(f)\right)=17-30 \log f
$$

Turbulence is the weakest noise source. It has minor influence in the very low frequency region and rapidly decays at $30 \mathrm{~dB} /$ decade. 
- Shipping noise

$$
10 \log \left(N_{s}(f)\right)=40+20(s-0.5)+26 \log (f)-60 \log (f+0.03)
$$

The noise caused by distant shipping is dominant in the frequency region from tens to hundreds of Hz. It is modeled through the shipping activity factor $s$, whose value ranges between 0 and 1 for low and high activity, respectively.

- Wave noise

$$
10 \log \left(N_{w}(f)\right)=50+7.5 \sqrt{w}+20 \log (f)-40 \log (f+0.4)
$$

where $w$ represents the wind speed in $\mathrm{m} / \mathrm{s}$. Surface motion is mainly caused by wind and, in fact, it represents the major contribution of site-specific noise in the region of interest for the underwater acoustic systems, i.e. from $100 \mathrm{~Hz}$ to $100 \mathrm{kHz}$.

The overall p.s.d. of the site-specific noise derives from the sum of each noise contribution

$$
N(f)=N_{t h}(f)+N_{t}(f)+N_{s}(f)+N_{w}(f)
$$

which shows a constant decay as frequency increases, thus it can be easily approximated within the region of interest $(f<50 \mathrm{kHz})$ as

$$
10 \log (N(f)) \approx N_{1}+\eta \log (f)
$$

The noise p.s.d. is illustrated in Fig.1.2 for different values of ship activity and different wind speeds. The dominant noise source in each region can be easily identified from the figure: ship activity strongly increases the noise between $10 \mathrm{~Hz}$ and $100 \mathrm{~Hz}$, where the wind speed does not have any effect, and the opposite happens at the $100 \mathrm{~Hz}-10 \mathrm{kHz}$ region. The approximation is also shown in figure 1.3 with $N_{1}=50 \mathrm{~dB}$ re $\mu \mathrm{Pa}$ and $\eta=18 \mathrm{~dB} /$ decade.

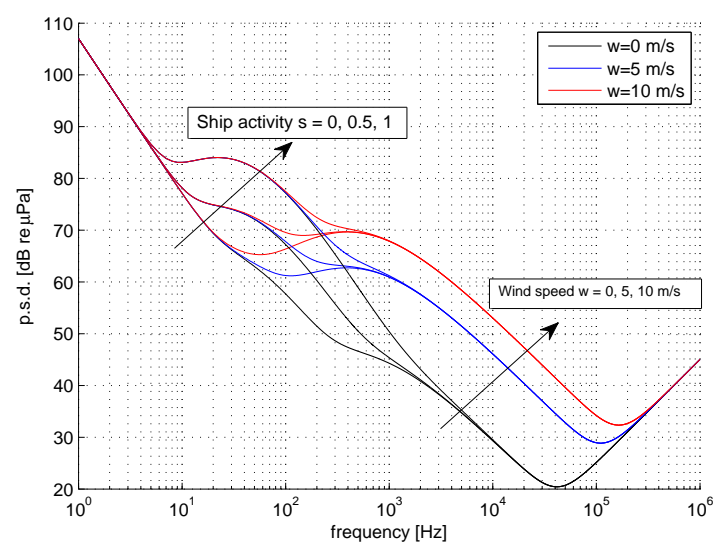

Figure 1.2: Site-specific noise p.s.d.

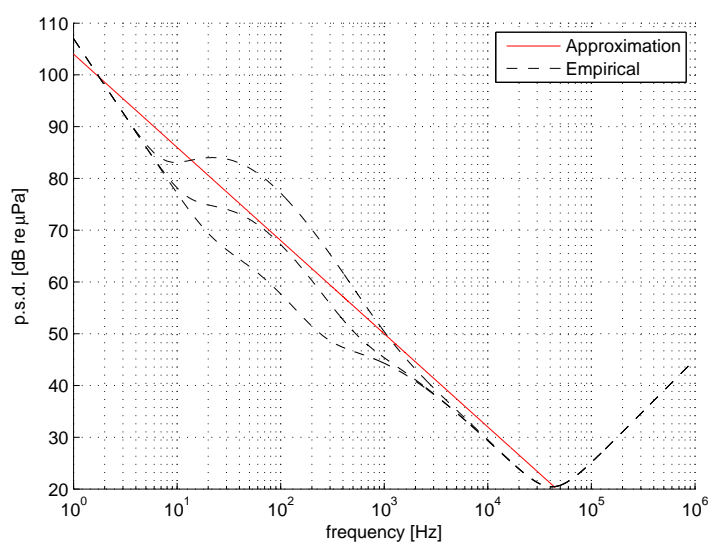

Figure 1.3: Noise p.s.d with approximation. 


\section{Channel bandwidth}

Once the attenuation $A(l, f)$ and the noise p.s.d. $N(f)$ are defined, one can evaluate the signalto-noise ratio observed at the receiver over a distance $l$. Without taking into account additional losses such as directivity, shadowing, etc., the narrow-band SNR is given by

$$
\operatorname{SNR}(l, f)=\frac{S_{l}(f)}{A(l, f) N(f)}
$$

where $S_{l}(f)$ is the power spectral density of the transmitted signal. From Fig.1.4 is evident that the acoustic $3 \mathrm{~dB}$ bandwidth depends on the transmission distance, since the narrow-band SNR function given by (1.10) is different for any given $l$. The frequency at which the attenuation is minimum is denoted as $f_{0}(l)$. The $3 \mathrm{~dB}$ bandwidth $B_{3}(l)$ is defined as the range of frequencies around $f_{0}(l)$ over which $\operatorname{SNR}(l, f)>\operatorname{SNR}\left(l, f_{0}(l)\right) / 2$. The solid lines in the figure represent the bandwidth $B_{3}(l)$ for the case where the transmitted signal p.s.d. is flat, i.e. $S_{l}(f)=S_{l}$. Figure 1.4

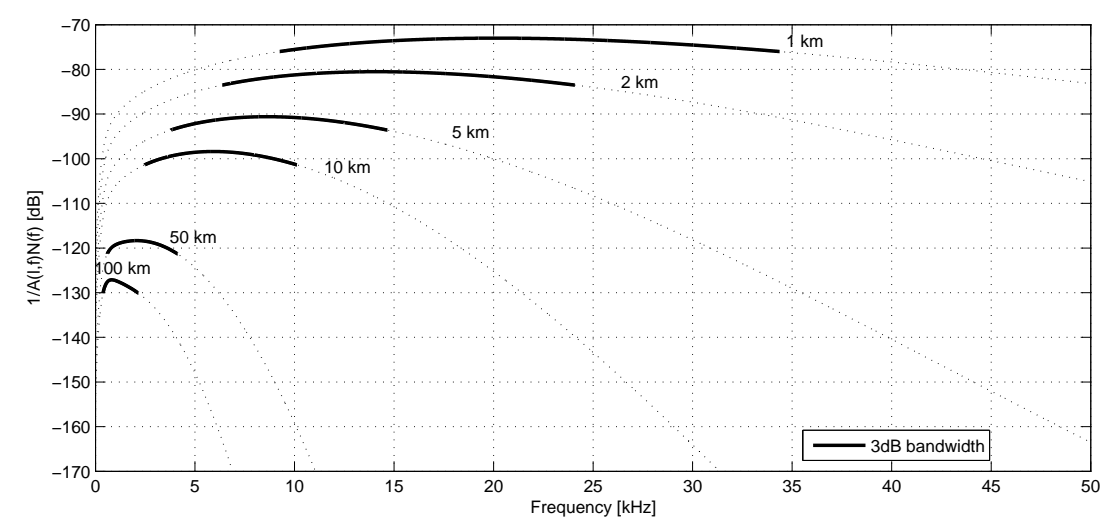

Figure 1.4: Evaluation of $1 / A(l, f) N(f)$ for spreading factor $k=1.5$, moderate shipping activity $(s=0.5)$, no wind $(w=0 \mathrm{~m} / \mathrm{s})$ and distances $l=\{1,2,5,10,50,100\} \mathrm{km}$

may also be used as a reference for the design parameters of an underwater communication system. If the distances over which the system will communicate are known a priori, one can effectively allocate the transmission power over the optimal frequencies so as to achieve the maximum SNR. For instance, if the transmission is to be conducted over a distance of, say 1 or $2 \mathrm{~km}$, the best transmission range is over 10-25 kHz, while for longer distances (50 to $100 \mathrm{~km}$ ) one should not use frequencies over $5 \mathrm{kHz}$. This trend indicates that both the optimal frequency and the available bandwidth become smaller as the distance increases, see Fig.1.5.

\section{Resource allocation}

There are many criteria to allocate the transmitted power in a given bandwidth [11]. For instance, the simplest case is when a flat p.s.d. is employed for transmission. In this case one sets the bandwidth to some $B(l)=\left[f_{\min }(l), f_{\max }(l)\right]$ around $f_{0}(l)$, and adjusts the transmission power 


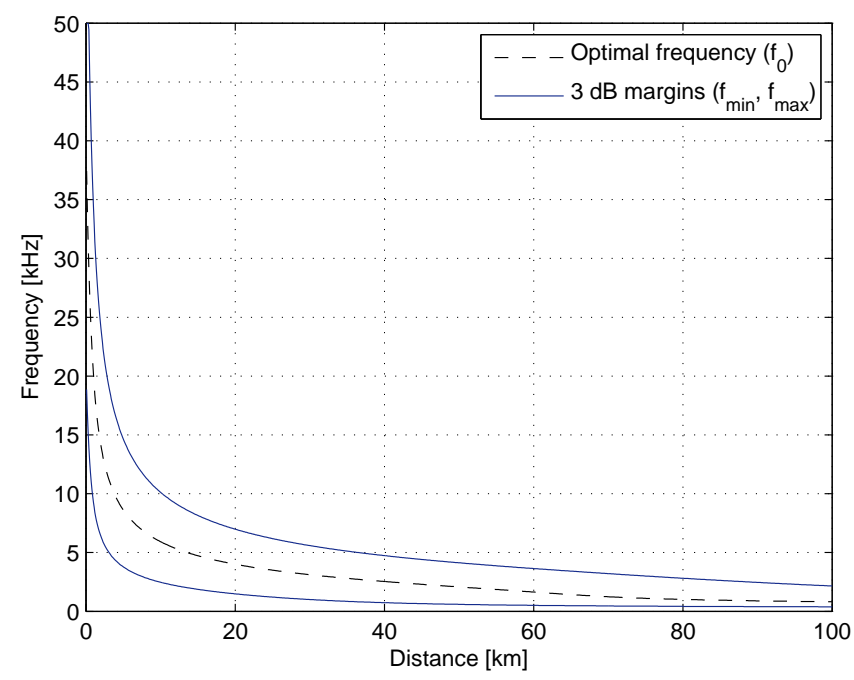

Figure 1.5: Optimal frequency $f_{0}$ and $3 \mathrm{~dB}$ bandwidth margins as a function of distance.

$P(l)$ to achieve the desired total SNR, which we will call $\mathrm{SNR}_{0}$. From the definition of power spectral density we have that

$$
P(l)=\int_{B(l)} S_{l}(f) d f
$$

and the total SNR is given by

$$
\operatorname{SNR}(l, B(l))=\frac{\int_{B(l)} S_{l}(f) A^{-1}(l, f) d f}{\int_{B(l)} N(f) d f}
$$

Considering the case where $S_{l}(f)$ is constant, the result of $(1.11)$ is $S_{l}(f)=P(l) / B(l)$ and, consequently, (1.12) reduces to a closed form expression, which determines the power to be transmitted as a function of the target SNR

$$
P(l)=\mathrm{SNR}_{0} B(l) \frac{\int_{B(l)} N(f) d f}{\int_{B(l)} A^{-1}(l, f) d f}
$$

and plugging into (1.11), we finally obtain

$$
S_{l}(f)= \begin{cases}S N R_{0} \frac{\int_{B(l)} N(f) d f}{\int_{B(l)} A^{-1}(l, f) d f} & \text { if } f \in B(l) \\ 0 & \text { otherwise }\end{cases}
$$

In general, however, one may take advantage of the power allocation to maximize a performance metric, such as the channel capacity. Assuming that the total bandwidth can be divided into many narrow sub-bands, the capacity can be obtained as the sum of the individual capacities. The $i$-th narrow sub-band is centered around the frequency $f_{i}$ and has a width $\Delta f$, which is considered to be small enough such that: 
- The channel transfer function appears frequency non-selective.

- The noise in this sub-band is white with p.s.d. $N\left(f_{i}\right)$.

- The only distortion comes from a constant attenuation factor $A\left(l, f_{i}\right)$.

Leveraging on these assumptions we define the resulting capacity as

$$
C(l)=\sum_{i} \Delta f \log _{2}\left[1+\frac{S_{l}\left(f_{i}\right) A^{-1}\left(l, f_{i}\right)}{N\left(f_{i}\right)}\right]
$$

Maximizing the capacity with respect to $S_{l}(f)$, subject to the constraint that the total transmitted power is finite, yields the optimal energy distribution. Unlike in eq.(1.13), the signal p.s.d. cannot be obtained in a closed form solution and the maximization problem is usually solved with the water-filling principle, which is described in Sec.3.2.2.

\subsection{Multipath channel}

Multipath propagation is one of the most common problems in wireless communications. The multipath propagation phenomenon occurs when communication signals arrive at the receiver from two or more paths with different delays. The occurrence of multipath propagation in the ocean is governed by two effects: sound refraction (Fig.1.7), and sound reflection at the surface, bottom or any close objects (Fig.1.6). The sound refraction is produced by the variation of the sound speed as a function of the depth. This speed profile creates a gradient of the refraction index and traps the acoustic waves that emerge from the source, similarly as in fiber-optic communications. This effect is mostly evident in deep water channels.

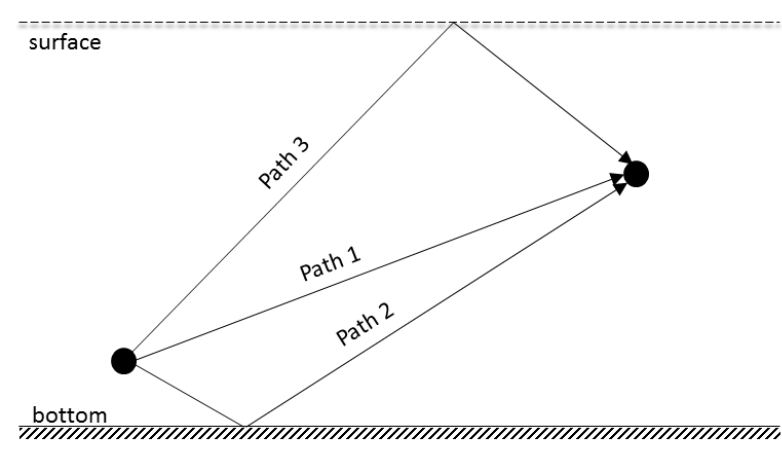

Figure 1.6: Reflection multi-path formation.

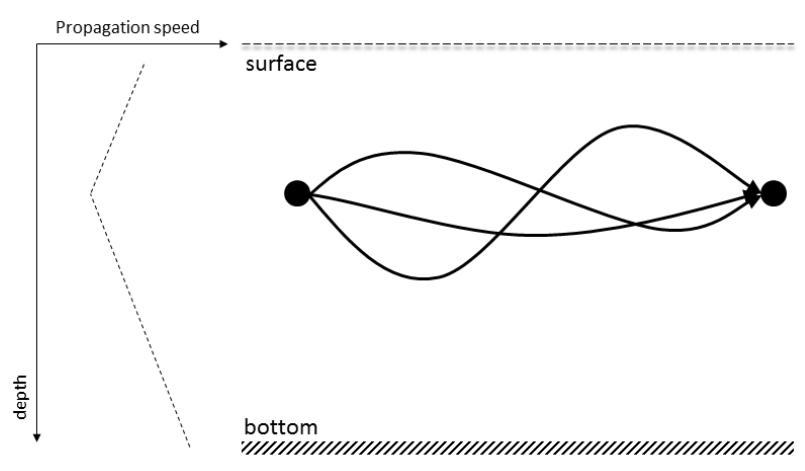

Figure 1.7: Acoustic wave refraction.

The impulse response is thus defined by the channel geometry and its reflection properties, which determine the number of paths and the gain associated with each arrival. To model the channel, we denote by $l_{p}$ the length traversed by the $p$-th path, where $p=0$ corresponds to the first arrival. In shallow water, the propagation speed can be considered constant and the path delays can be obtained as $l_{p} / c$. The reference time at the receiver $t_{0}$ is inferred from the first 
arrival and the relative path delays are then defined as $\tau_{p}=l_{p} / c-t_{0}$. The path gains depend on the cumulative reflection coefficient $\Gamma_{p}$ and the previously defined propagation loss $A_{p}\left(l_{p}, f\right)$. Consequently, each path has an associated function of frequency, namely a low pass filter. The overall channel response in frequency domain is equivalent to the sum of the responses of each individual path, which are $H_{p}(f)=\Gamma_{p} / \sqrt{A_{p}\left(l_{p}, f\right)}$. The frequency response is thus expressed as

$$
H(f)=\sum_{p} H_{p}(f) e^{-\mathrm{j} 2 \pi f \tau_{p}}
$$

and the corresponding impulse response is

$$
h(\tau)=\sum_{p} h_{p}\left(\tau-\tau_{p}\right)
$$

where $h_{p}(\tau)$ is the inverse Fourier transform of $H_{p}(f)$.

\section{Channel model for multi-carrier systems}

An effective approach to overcome the frequency selective distortion produced by the multipath propagation is through employing multi-carrier modulation. Considering an OFDM system, the transmitted signal on the $k$-th subcarrier of frequency $f_{k}=f_{0}+k \Delta f$ is given by

$$
s_{k}(t)=R e\left\{d_{k} g(t) e^{\mathrm{j} 2 \pi f_{k} t}\right\} \quad k=0 \ldots K-1
$$

where $d_{k}$ is the data symbol and $g(t)$ is a shaping pulse of duration $T=1 / \Delta f$. The broadband signal occupies a total bandwidth $B=K \Delta f$ consisting of the $K$ narrowband carriers

$$
s(t)=\sum_{k=0}^{K-1} s_{k}(t)=\operatorname{Re}\left\{u(t) e^{\mathrm{j} 2 \pi f_{0} t}\right\}
$$

where $u(t)=\sum_{k} d_{k} g(t) e^{\mathrm{j} 2 \pi k \Delta f t}$ is the baseband equivalent signal. According to the design specifications of the OFDM modulation, now one can assume that $\Delta f$ is indeed small enough such that the path response is flat in each sub-carrier, i.e. $H_{p}(f) \approx H_{p}\left(f_{k}\right)$ for $f \in\left[f_{k}-\Delta f / 2, f_{k}+\Delta f / 2\right]$. Therefore, the received signal is

$$
r(t)=\sum_{k=0}^{K-1} \sum_{p} H_{p}\left(f_{k}\right) s_{k}\left(t-\tau_{p}\right)
$$

A simpler model arises when the coefficients $H_{p}\left(f_{k}\right)$ are considered independent of $k$, i.e. the path transfer functions are flat over the entire bandwidth $B$. Then, the channel response simplifies to

$$
h(\tau)=\sum_{p} h_{p} \delta\left(\tau-\tau_{p}\right)
$$


Although this assumption does not exactly hold for the broadband acoustic system, it is often considered for its reduced complexity.

\subsection{The Doppler effect}

The Doppler effect is present when a relative velocity $v$ exists between the transmitter and receiver, i.e. $|v|>0$. The magnitude of the Doppler effect is proportional to $a=v / c$, and the distortion produced in underwater environments is high due to the low speed of sound $c=1500 \mathrm{~m} / \mathrm{s}$. For instance, the wave propagation speed in radiocommunications is $c_{0}=3 \cdot 10^{8} \mathrm{~m} / \mathrm{s}$. Hence, the Doppler effect experienced by a source moving at a relative velocity $v=1.5 \mathrm{~m} / \mathrm{s}$ is $a=$ $5 \cdot 10^{-9}$. Similar relative velocities are commonly observed in UWA communications even without intentional motion, i.e. underwater instruments are subject to drifting with waves, currents and tides, which, in this case, would produce a distortion five orders of magnitude greater, yielding a Doppler factor $a \sim 10^{-3}$. The Doppler distortion basically causes two effects: frequency spreading and frequency shifting. Such effects cannot to be neglected since they produce extremely high shifts that may eventually exceed the carrier spacing $\Delta f$.

The effect produced by Doppler distortion is inherently modeled into the signal delay. Let $g(t)$ be an arbitrary signal with period $T$, modulated onto a carrier of frequency $f_{c}$ and transmitted through an ideal channel. The relative velocity between the transmitter and the receiver is $v(t)$. In such a case, one can consider that the receiver has a different time scale $t_{R}=t-\frac{l_{0}-v(t) t}{c}$ due to the Doppler compression/dilatation. The received signal is then expressed as

$$
r(t)=\operatorname{Re}\left\{g\left(t_{R}\right) e^{\mathrm{j} 2 \pi f_{c} t_{R}}\right\}=\operatorname{Re}\left\{g\left(t+a(t) t-\tau_{0}\right) e^{\mathrm{j} 2 \pi f_{c}\left(t+a(t) t-\tau_{0}\right)}\right\}
$$

where $l_{0}$ is the distance at the instant $t_{0}$, and $\tau_{0}=l_{0} / c$. We will further assume that $v(t)$ is constant during the transmission of $g(t)$, i.e. that $a(t)=a=v / c$ for $t=(k-1) T, \ldots, k T, k \in \mathbb{N}$.

In frequency domain the signal is distorted in two ways. First, equivalently to the time scaling $T /(1+a)$, its bandwidth $B$ is observed as $B(1+a)$. Second, a frequency offset $a f_{c}$ is introduced. These effects are called Doppler spreading and Doppler shifting, respectively, and both can be identified in the baseband received signal

$$
b(t)=e^{-\mathrm{j} 2 \pi f_{c} \tau_{0}} g(\underbrace{t(1+a)}_{\text {spreading }}-\tau_{0}) \underbrace{e^{\mathrm{j} 2 \pi a f_{c} t}}_{\text {shifting }}
$$

In underwater acoustic communications, unlike in electromagnetic radiocommunications, the frequency shift cannot be considered equal for all sub-carriers. Consequently, this causes nonuniform frequency shifting, as illustrated in Fig. 1.8.

General bibliography for this chapter: [10, 12, 13]. 


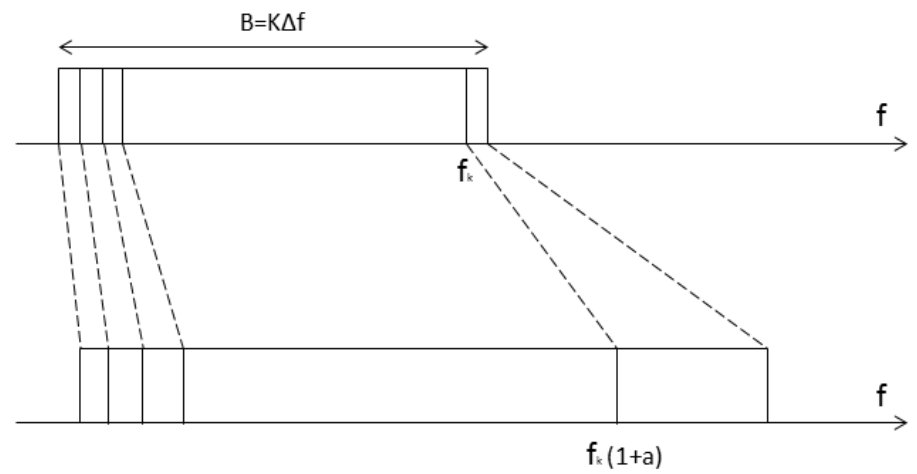

Figure 1.8: Non-uniform frequency shifting in a wideband system, caused by motion-induced Doppler distortion. 


\section{Chapter 2}

\section{Orthogonal Frequency Division Multiplexing}

As we observed in Chapter 1, the UWA channel is a nonideal linear filter. Therefore, if a bitrate of a few kbps were to be transmitted over an UWA channel, the use of single-carrier modulations would lead to severe inter-symbol interference (ISI). The ISI arises when the transmitted symbol period $T$ (a few ms for bitrates of kbps) is not much larger than the channel delay spread $T_{m p}$ (typically tens of milliseconds in an UWA channel), and causes a severe degradation of performance as compared with the ideal (flat) channel. The degree of performance degradation depends on the frequency response characteristics. Furthermore, in single-carrier modulations the complexity of the receiver's equalizer rapidly increases as the span of the ISI grows.

The orthogonal frequency division multiplexing (OFDM) is mainly considered for frequencyselective channels as it offers low complexity fast Fourier transform (FFT)-based signal processing, and ease of reconfiguration for use with different bandwidths. OFDM relies on the idea of dividing the available channel into a number of narrow subchannels, such that each subchannel is orthogonal to all the other one. The number of subchannels is chosen to yield a sufficiently small spacing $\Delta f$, such that the frequency response in each carrier can be considered flat. In an OFDM system, each subchannel is considered ideal and processed independently from the others, making equalization unnecessary. In addition, by virtue of having a narrowband signal on each carrier, OFDM is easily conducive to multi-input multi-output (MIMO) system configurations.

\subsection{Principles of operation}

\section{Orthogonality}

In an OFDM system, each of the $K$ modulated symbols (QPSK, 8-PSK, 16-QAM...), are translated into different equally spaced frequencies. The symbols are inserted every $\Delta f \mathrm{~Hz}$ until the entire bandwidth is occupied. The main advantage of OFDM is the low complexity required both at the transmitter and receiver stages. The use of narrow subbands avoids the need for complex 
equalization stages in time domain. Similarly, one can avoid dealing with inter-carrier interference (ICI) at the receiver if the modulation frequencies are orthogonal to one another. To achieve this goal, the frequency spacing needs to be defined accordingly. Let us consider that the symbol $d_{k}(n)$ is to be transmitted on the $k$-th subcarrier during the $n$-th OFDM symbol, then

$$
s_{k, n}(t)=d_{k}(n) e^{-\mathrm{j} 2 \pi k \Delta f t} \quad t=n T, \ldots,(n+1) T
$$

the OFDM symbol is thus obtained as the sum of the $K$ subcarriers

$$
s_{n}(t)=\sum_{k=0}^{K-1} d_{k}(n) e^{\mathrm{j} 2 \pi k \Delta f t} \quad t=n T, \ldots,(n+1) T
$$

We want to determine $\Delta f$ such that each subband does not interfere with the rest. To do so, it is necessary to evaluate the orthogonality between two arbitrary subcarriers $k$ and $m$

$$
\begin{aligned}
\left\langle s_{k}(t), s_{m}(t)\right\rangle & =d_{k}(n) d_{m}^{*}(n) \frac{1}{T} \int_{n T}^{(n+1) T} e^{\mathrm{j} 2 \pi(k-m) \Delta f t} d t= \\
& =d_{k}(n) d_{m}^{*}(n) \frac{e^{\mathrm{j} 2 \pi(k-m) \Delta f(n+1) T}-e^{\mathrm{j} 2 \pi(k-m) \Delta f n T}}{\mathrm{j} 2 \pi(k-m) \Delta f T}= \\
& =d_{k}(n) d_{m}^{*}(n) e^{\mathrm{j} 2 \pi(k-m) \Delta f n T} \frac{e^{\mathrm{j} 2 \pi(k-m) \Delta f T}-1}{\mathrm{j} 2 \pi(k-m) \Delta f T}= \\
& =C \cdot \operatorname{sinc}((k-m) \Delta f T)
\end{aligned}
$$

where $C=d_{k}(n) d_{m}^{*}(n) e^{\mathrm{j} \pi(k-m) \Delta f(2 n+1) T}$. The condition for orthogonality between subcarriers implies that $\left\langle s_{k}(t), s_{m}(t)\right\rangle=\delta(k-m)$, hence, we set

$$
C \cdot \operatorname{sinc}((k-m) \Delta f T)=\delta(k-m) \quad \Rightarrow \quad \Delta f T=n, \quad n \in \mathbb{N}_{1}
$$

therefore if unit-energy symbols are employed, it is easy to see that $C=1$ when $k=m$. The so-obtained result indicates that only certain values of $\Delta f$ maintain the orthogonality between subcarriers, and those values depend on the symbol length $T$. When the system is to be designed, one usually has knowledge of the transmission bandwidth $B$, so it is easier to first determine $\Delta f$ (or $K$ ) that best fits into the available bandwidth and then the symbol duration can be determined accordingly as

$$
T=1 / \Delta f=K / B
$$

\section{Mathematical description}

In the above section we have determined that the essence of OFDM relies on modulating the symbols to orthogonal frequencies to further simplify the operations at the receiver. The system is fully defined by the bandwidth $B$ and the number of subcarriers $K$, which are equally spaced at $\Delta f=B / K$. The symbol duration is $T=1 / \Delta f$ and a guard interval of duration $T_{g}$, sufficient to 
accommodate the multipath spread $T_{m p}$, is appended for the total block duration of $T^{\prime}=T+T_{g}$. If $d_{k}(n)$ is the symbol modulated onto the $k$-th carrier during the $n$-th block, the OFDM signal is expressed as

$$
s(t)=\sum_{n=0}^{N-1} \sum_{k=0}^{K-1} d_{k}[n] e^{\mathrm{j} 2 \pi\left(f_{0}+k \Delta f\right) t} \prod\left(\frac{t-n T^{\prime}}{T}\right)
$$

where $f_{0}$ is the frequency of the first carrier. Note that the orthogonality between carriers is clear when we calculate the Fourier transform of (2.6)

$$
S_{n}(f)=\int_{n T^{\prime}}^{(n+1) T^{\prime}} s(t) e^{-\mathrm{j} 2 \pi f t} d t=T \sum_{k=0}^{K-1} d_{k}[n] e^{-\mathrm{j} 2 \pi\left(f-f_{0}-k \Delta f\right)\left(n T^{\prime}+T / 2\right)} \operatorname{sinc}\left(\frac{f-f_{0}-k \Delta f}{\Delta f}\right)
$$

The bandwidth efficiency, defined as the number of bits per second per Hertz (bps/Hz), is a measure of how efficiently a limited frequency spectrum is utilized by the modulation

$$
\frac{R}{B}=\frac{\alpha M K / T^{\prime}}{B}=\frac{\alpha M}{1+T_{g} B / K} \quad[\mathrm{bps} / \mathrm{Hz}]
$$

where $\alpha$ is the code rate and $M$ is the number of bits per symbol provided by the modulation, i.e. 2 bits/symbol for quadrature amplitude modulation (QAM), 4 bits/symbol 16-QAM, etc. In an ideal non-selective channel, where the guard interval could be set $T_{g}=0$, the bandwidth efficiency does not depend on $K$. However, given that OFDM is meant to deal with frequencyselective channels, $T_{g}$ in UWA communications is usually on the same order of magnitude as $T$. In this case, one can see that the bandwidth efficiency rapidly increases as more carriers are packed within the given bandwidth. The bandwidth efficiency can also be increased by using higher order modulations, such as 64-QAM. If one expects to have a slowly varying but noisy channel, small $M$ and large $K$ are more appropriate. On the contrary, on rapidly varying channels with good SNR, one may prefer to reduce the number of carriers and increase the number of bits per symbol, i.e. use a modulation of higher order.

An OFDM signal as a function of time is illustrated in Fig.2.1. This signal bears $K=8$ QPSK carriers distributed within a bandwidth $B=1 \mathrm{kHz}$. This corresponds to a frequency spacing of $\Delta f=125 \mathrm{~Hz}$ and a symbol length of $T=8 \mathrm{~ms}$. The guard interval is $T_{g}=10 \mathrm{~ms}$ and the first carrier frequency $f_{0}=5 \mathrm{kHz}$. Assuming a code rate of $\alpha=1$ (uncoded), the bandwidth efficiency is $0.89 \mathrm{bps} / \mathrm{Hz}(1.52 \mathrm{bps} / \mathrm{Hz}$ for $K=32$ and $1.86 \mathrm{bps} / \mathrm{Hz}$ for $K=128)$. The spectrum of each OFDM carrier is shown in Fig.2.2.

When the signal reaches the receiver, it has been altered by the communication channel. Independently of the channel model used, the received signal $r(t)$ can be modeled as

$$
r(t)=s(t) * h(t, \tau)+z(t)
$$

where $z(t)$ is zero-mean additive noise. The signal processing at the receiver consists of carrying 

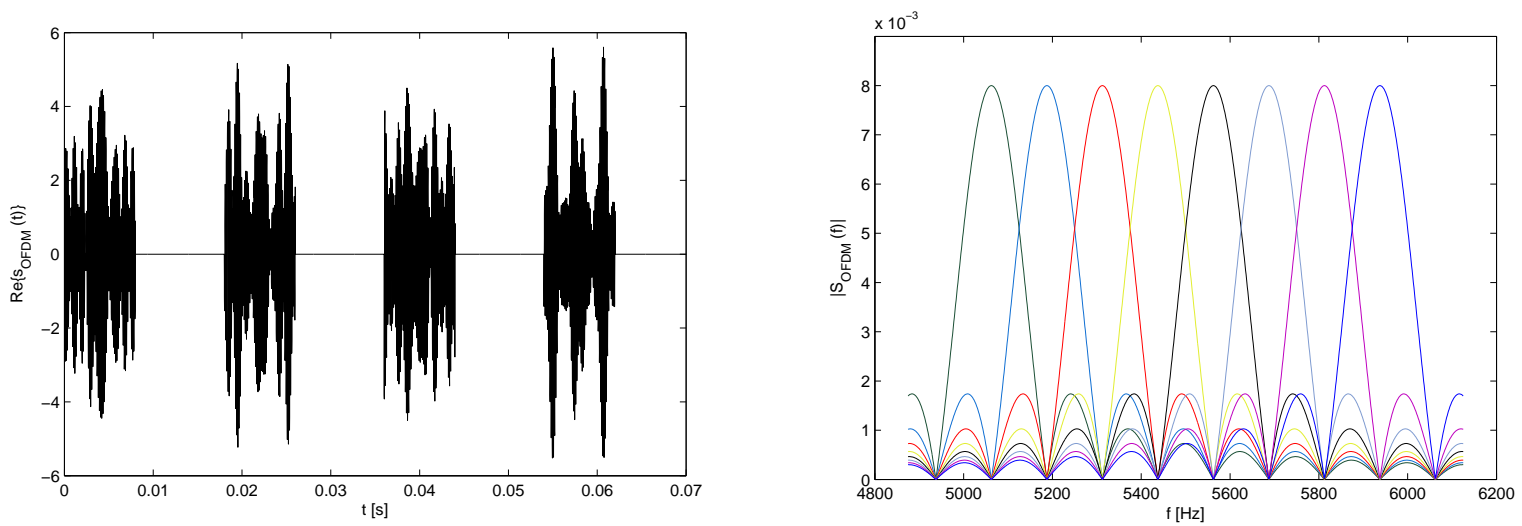

Figure 2.1: OFDM signal. $K=8, B=1 \mathrm{kHz}$, Figure 2.2: Spectrum of an OFDM signal. $K=$ $T_{g}=10 \mathrm{~ms}$. $8, B=1 \mathrm{kHz}, f_{0}=5 \mathrm{kHz}$.

out the following integration to recover the $m$-th carrier of the $n$-th OFDM block

$$
y_{n, m}(t)=\int_{n T^{\prime}}^{(n+1) T^{\prime}} r(t) e^{-\mathrm{j} 2 \pi\left(f_{0}+m \Delta f\right) t} d t=\int_{n T^{\prime}}^{(n+1) T^{\prime}}(s(t) * h(t, \tau)+z(t)) e^{-\mathrm{j} 2 \pi\left(f_{0}+m \Delta f\right) t} d t
$$

Assuming that the channel stays constant during the OFDM block, i.e. $h(t, \tau)=h(\tau)$, and using the following Fourier transform properties

$$
\begin{aligned}
\mathcal{F}\{x(t) * y(t)\} & =\mathcal{F}\{x(t)\} \cdot \mathcal{F}\{y(t)\} \\
\left.\mathcal{F}\{x(t)\}\right|_{f=f_{0}} & =\int_{-\infty}^{\infty} x(t) e^{-j 2 \pi f_{0} t} d t
\end{aligned}
$$

we have that

$$
y_{n, m}(t)=H\left(f_{0}+m \Delta f\right) \cdot S_{n}\left(f_{0}+m \Delta f\right)+\tilde{z}
$$

Recall from (2.7) that $S_{n}\left(f_{0}+m \Delta f\right)=d_{m}(n)$, so

$$
y_{n, m}(t)=H\left(f_{0}+m \Delta f\right) \cdot d_{m}(n)+\tilde{z}
$$

where $\tilde{z}$ is still zero-mean additive noise.

\section{FFT implementation}

Imagine that we want to implement the OFDM modulation in a digital signal processor (DSP) that uses a certain sampling frequency $f_{s}$. For simplicity, and to allow further Doppler compensation at the receiver, the signal bandwidth $B$ is chosen as a divisor of $f_{s}$, i.e. $f_{s}=Q \cdot B$. Typical values of $Q$ that provide enough resolution for the resampling algorithm are 4 or 8 . To allocate $K$ carriers within the desired bandwidth $B$, we will need $Q \cdot K$ samples in total. To generate the 
OFDM signal, we first pack the data to be transmitted into a column vector

$$
\mathbf{d}(n)=\left[\begin{array}{llll}
d_{0}(n) & d_{1}(n) & \cdots & d_{K-1}(n)
\end{array}\right]^{T}
$$

Now we follow equation (2.6), which is exactly the inverse discrete Fourier transform (IDFT) of $\mathbf{d}(\mathrm{n})$, so we directly compute $\mathbf{s}(\mathrm{n})$ as

$$
\mathbf{s}(n)=\left[\begin{array}{llll}
s\left(n T^{\prime}\right) & s\left(n T^{\prime}+1 / f_{s}\right) & \ldots & s\left(n T^{\prime}+(Q K-1) / f_{s}\right)
\end{array}\right]^{T}=\frac{1}{Q K} \mathbf{F d}(n)
$$

where

$$
\mathbf{F}=\left[\begin{array}{ccccc}
1 & 1 & 1 & \ldots & 1 \\
1 & e^{\mathrm{j} 2 \pi \frac{1}{Q K}} & e^{\mathrm{j} 2 \pi \frac{2}{Q K}} & \ldots & e^{\mathrm{j} 2 \pi \frac{K-1}{Q K}} \\
1 & e^{\mathrm{j} 2 \pi \frac{2}{Q K}} & e^{\mathrm{j} 2 \pi \frac{4}{Q K}} & \ldots & e^{\mathrm{j} 2 \pi \frac{2(K-1)}{Q K}} \\
\vdots & \vdots & \vdots & & \vdots \\
1 & e^{\mathrm{j} 2 \pi \frac{Q K-1}{Q K}} & e^{\mathrm{j} 2 \pi \frac{(Q K-1) 2}{Q K}} & \ldots & e^{\mathrm{j} 2 \pi \frac{(Q K-1)(K-1)}{Q K}}
\end{array}\right]
$$

is a truncated $Q K \times K$ IDFT matrix. The inverse matrix is used by the receiver, similarly as in (2.10) we have

$$
\mathbf{y}(n)=\mathbf{F}^{H} \mathbf{r}(n)
$$

To exploit the benefits of the FFT, one must select $K$ as a multiple of 2. Most FFT algorithms, such as divide and conquer [14], reduce the complexity of a $N$-point DFT from $O\left\{N^{2}\right\}$ to $O\left\{N \log _{2} N\right\}$ operations.

\section{Frequency selectivity}

In this section we will investigate the conditions in which the flat channel assumption is indeed reasonable. To do so, let us consider the channel model presented in (1.16). The relative delays $\tau_{p}^{\prime}=\tau_{p}-\tau_{0}$ can be replaced in the equation, so as to obtain

$$
\begin{aligned}
H\left(f_{k}\right) & =e^{-\mathrm{j} 2 \pi f_{k} \tau_{0}} \sum_{p} H_{p}\left(f_{k}\right) e^{-\mathrm{j} 2 \pi f_{k} \tau_{p}^{\prime}} \\
H\left(f_{k+1}\right) & =e^{-\mathrm{j} 2 \pi\left(f_{k}+\Delta f\right) \tau_{0}} \sum_{p} H_{p}\left(f_{k+1}\right) e^{-\mathrm{j} 2 \pi f_{k} \tau_{p}^{\prime}} e^{-\mathrm{j} 2 \pi \Delta f \tau_{p}^{\prime}}
\end{aligned}
$$

When the gap between carriers is sufficiently small, it is reasonable to assume that the coefficients $H_{p}\left(f_{k}\right) \approx H_{p}\left(f_{k+1}\right)$, i.e. the UWA channel attenuation is insignificant between two consecutive carriers (Sec.1.2). More concretely, we consider that the channel is flat if $\left|H\left(f_{k}\right)\right| \approx\left|H\left(f_{k+1}\right)\right|$, therefore

$$
\left|\sum_{p} H_{p}\left(f_{k}\right) e^{-\mathrm{j} 2 \pi f_{k} \tau_{p}^{\prime}}\right| \approx\left|\sum_{p} H_{p}\left(f_{k}\right) e^{-\mathrm{j} 2 \pi f_{k} \tau_{p}^{\prime}} \cdot e^{-\mathrm{j} 2 \pi \Delta f \tau_{p}^{\prime}}\right|
$$


and the equation holds if

$$
e^{-\mathrm{j} 2 \pi \Delta f \tau_{p}^{\prime}}=1 \quad \Rightarrow \quad \Delta f \tau_{p}^{\prime} \ll 1 \quad \Rightarrow \quad T_{m p} \ll T
$$

i.e., the block length has to be much larger than the channel delay spread.

\subsection{OFDM transmitter and receiver}

In this section we will explain in detail the structure of a generic OFDM system. The system model flowchart of the transmitter is shown in Fig. 2.3. An uncorrelated bit stream enters the system from the left input and is processed throughout the following blocks to finally obtain the OFDM symbol at the right end of the diagram.

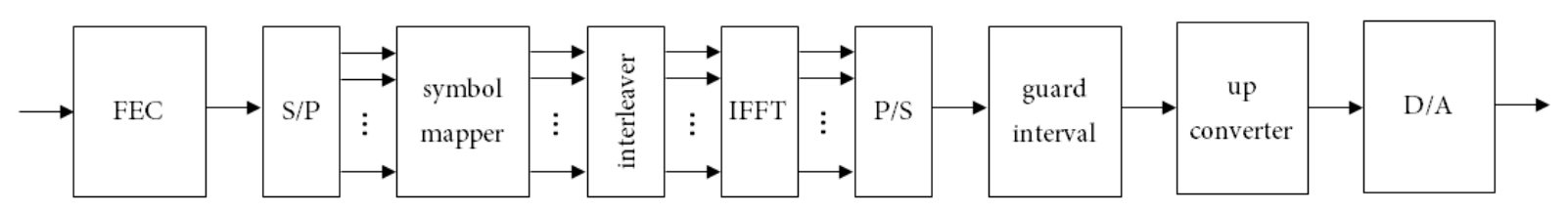

Figure 2.3: Block diagram of an OFDM transmitter.

The block diagram for the receiver is shown in Fig. 2.4. The blocks that involve specific signal processing (synchronization, resampling and detection) may change in depending on the application as will be described in Chapter 4 .

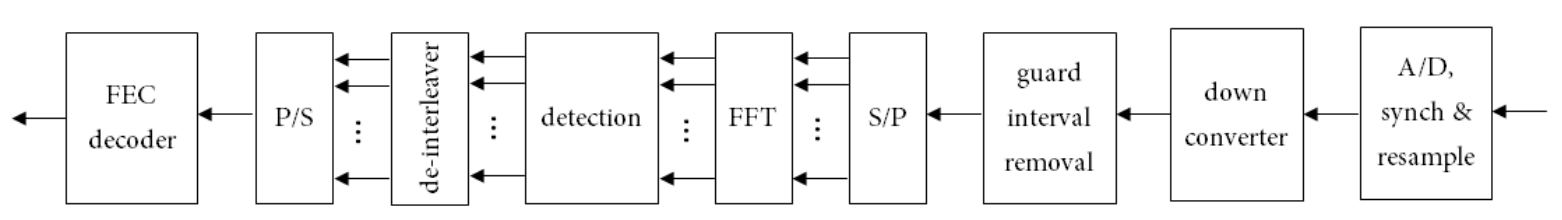

Figure 2.4: Block diagram of an OFDM receiver.

\section{Forward error correction code}

The forward error correction (FEC), or channel coding, is a technique used to detect and/or correct errors in data transmission over unreliable or noisy communication channels. The main idea is to add redundancy to the data at the transmitter by using an error correction code (ECC). The redundancy allows the receiver to detect a limited number of errors that may occur during 
the transmission, which can usually be corrected without the need for retransmissions. The long packet delay due to the slow propagation speed, or the absence of a feedback link motivate the use of correction codes in underwater communications to avoid packet retransmissions.

There are two main categories of FEC codes:

1. Block codes usually work on blocks of bits of fixed and predetermined size. For instance, a $(15,11)$ block has a length of 15 bits and contains 11 data bits. Block codes can be generally decoded in polynomial time respective to their block length.

2. Convolutional codes work on bit or symbol streams of arbitrary length. The algorithm used for decoding is the Viterbi algorithm, which has an asymptotically optimal decoding efficiency as the length of the convolutional code increases. This decoding efficiency, however, is at the expense of an exponentially increasing complexity.

\section{Symbol mapping}

The symbol mapping block turns groups of bits into symbols that are allocated in each OFDM subcarrier. Each symbol is represented by a complex number, where the real and imaginary parts are denoted by in-phase and quadrature components, respectively. The symbol associated to each codeword of bits depends on the modulation scheme. The most common modulations are:

- Quadrature Amplitude Modulation (QAM) is a modulation where the constellation points are arranged in a square grid with equal vertical and horizontal spacing. In an MQAM modulation, the phase and quadrature components are quantized into $\log _{2}(M)$ levels, where $M$ is the cardinality of the symbol alphabet. Fig. 2.5 shows the constellation of a 16-QAM scheme.

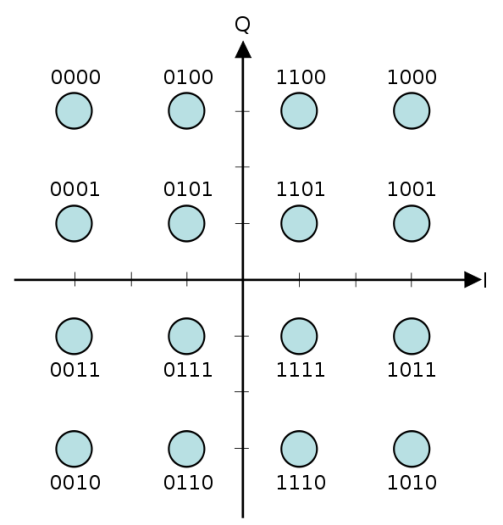

Figure 2.5: Constellation of a 16-QAM modulation scheme.

- Phase Shift Keying (PSK) arranges the constellation points in a circle of radius (norm) one. The symbols are placed with a constant and uniform phase difference. If $M$ is the cardinality of the alphabet, the symbols are spaced $2 \pi / M$ radians. Since all the symbols 
have the same norm, this modulation is especially appropriate for use with differential encoding. When the received phases are directly compared to reference symbols, the system is termed coherent. Alternatively, instead of the symbols themselves, the transmitter can initially send a known symbol and the successive phase changes with respect to the preceding symbols. This method can be significantly simpler to implement at the receiver and avoids the need for OFDM channel estimation. Fig. 2.6 shows the constellation of a 8-PSK scheme.

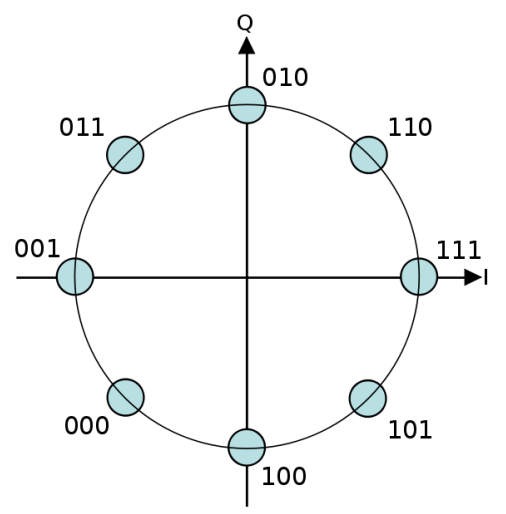

Figure 2.6: Constellation of a 8-PSK modulation scheme.

\section{Interleaving}

Frequency interleaving is commonly used in OFDM systems to improve the performance of forward error correction codes. This is done by separating the symbols of a codeword in the available bandwidth (across the $K$ subcarriers) as much as possible. The aim is to increase the correction probability of the ECC by eliminating error bursts inside the same codeword, which are produced by highly distorted or attenuated frequency bands. The interleaving scheme is shown in Fig. 2.7.

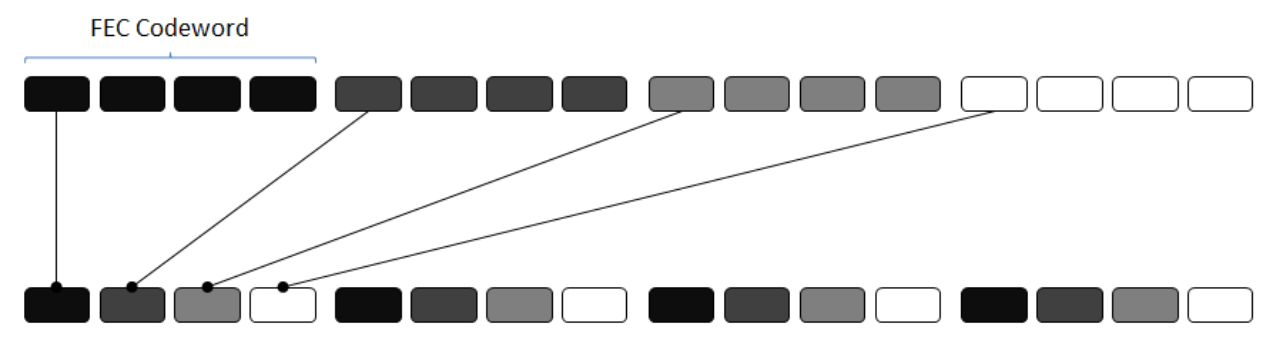

Figure 2.7: Interleaving scheme for an OFDM system. 


\subsection{Inter-carrier interference}

As it has been shown in Sec. 2.1, one of the main advantages of an OFDM system is the orthogonality between subcarriers, that allows for independent and fast data decoding. However, under certain channel conditions the orthogonality vanishes and a small contribution from all symbols appears in each subcarrier.

An important source of this self-interference, which is called inter-carrier interference (ICI), is the Doppler distortion. When there exists a relative motion between the two ends of the transmission, the frequency spectrum suffers from a non-uniform frequency shifting $(1+a) f$. Hence, the frequency spacing between carriers at the receiver can be characterized as

$$
\Delta f_{k}^{\prime}=f_{k+1}+a f_{k+1}-f_{k}-a f_{k}=\Delta f \cdot(1+a)
$$

which breaks the orthogonality condition (2.5). The interference level experienced depends on the Doppler shift, i.e. on the displacement of the carrier with respect to the original carrier on which the symbol is transmitted. When the shift is comparable to the frequency spacing, the neighboring symbols produce a strong interference, thus leading to unsuccessful decoding. In general, to compensate the Doppler shift, high resolution probe blocks are used to measure the Doppler factor that affects a certain number of OFDM symbols. In the figure 2.8 we show an example of ICI produced by Doppler shifting. The signal is OFDM with $B=7.5 \mathrm{kHz}, K=8$ carriers and a Doppler factor of $a=10^{-2}$. The received signal is represented by the dashed line, and the symbol contribution and the interference are represented by blue and red circles, respectively.
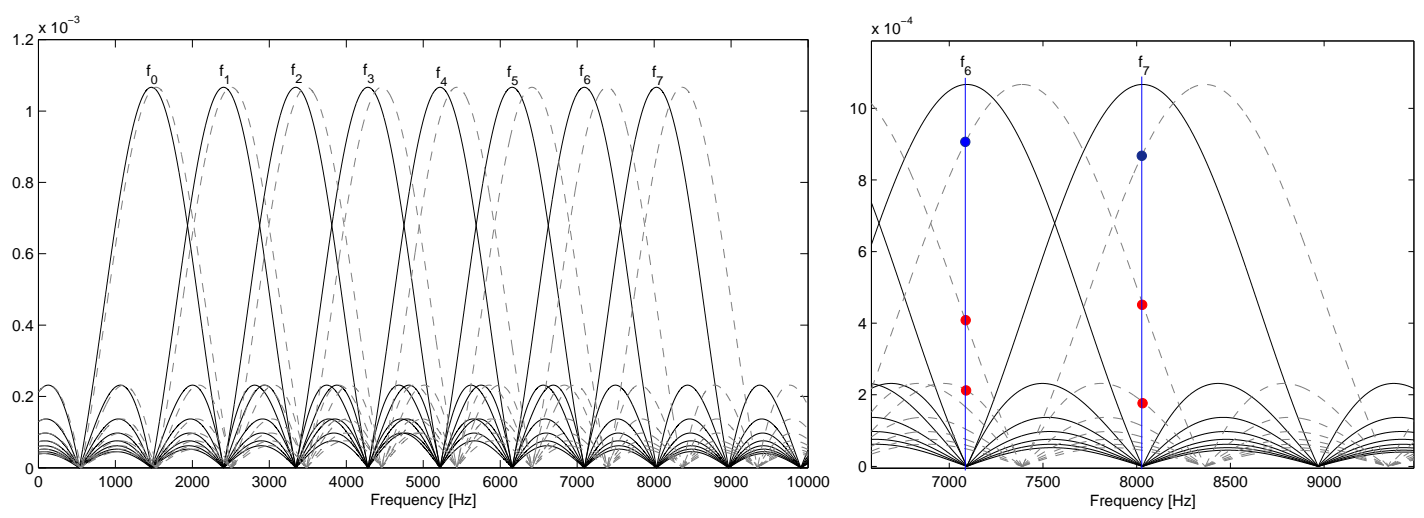

Figure 2.8: Inter-carrier interference produced by Doppler shifting.

Another important source of ICI is the channel time-variation. Ideally, the channel remains approximately constant during an OFDM block. When this condition is not satisfied, the orthogonality between subcarriers is lost. Hence, Doppler spread that affects the channel and, consequently, the channel coherence time, become critical. The shortage of available bandwidth motivates the use of small frequency spacing, which leads to OFDM symbol durations on the order 
of tens to hundreds of milliseconds. During this time, the channel can change noticeably and the time invariability assumption is no longer valid. In the experimental tests, the channel coherence time is shown to be on the same order as the block duration.

\subsection{System overview}

To summarize, the main advantages and drawbacks of an OFDM system are

\section{Advantages}

- High spectral efficiency.

- Multipath equalization without the need for complex filters.

- Robustness against frequency-selective fading.

- Robustness against intersymbol interference (ISI) with the use of a guard interval.

- Efficient and fast implementation using FFT.

- Easy to adapt to the channel conditions (number of carriers, guard interval, etc).

\section{Drawbacks}

- High sensitivity to frequency synchronization.

- Sensitivity to frequency shifts (Doppler).

- High peak-to-average power ratio (PAPR). 


\section{Chapter 3}

\section{Multiple-input multiple-output communications}

The use of multiple elements at the transmitter and the receiver in a wireless system, known as multi-input multi-output (MIMO) wireless communications, is an emerging technology that promises significant improvements in capacity, spectral efficiency and link reliability. The use of MIMO is especially convenient in underwater communications, because the mentioned gains are achieved simply by adding multiple receivers/transmitters and non-complex processing stages. Additionally, neither the bandwidth nor the transmission power need to be increased. In general, the MIMO systems are configured to provide either spatial multiplexing or diversity gain, however, we will briefly discuss certain coding schemes that can achieve both gains upon a diversitymultiplexing trade-off.

Under suitable fading channel conditions, having both multiple transmitters $\left(M_{T}\right)$ and receivers $\left(M_{R}\right)$ provides an additional spatial dimension for communication and yields a degreeof-freedom gain. These additional degrees of freedom can be exploited by spatially multiplexing several data streams onto the MIMO channel, which leads to a capacity increase proportional to $n=\min \left(M_{T}, M_{R}\right)$.

Alternatively, the MIMO configuration can be used to provide diversity gain, either in transmission, reception, or both. In this scheme, each transmitter-receiver pair ideally suffers from independent fading. The idea is to combine each channel contribution so as to obtain a resultant signal that exhibits considerably less amplitude variability, as compared with the signal at any one receiving element. Such scheme has a maximum achievable diversity gain of $M_{T} \cdot M_{R}$. The precise goal of this work is to design a transmission and a reception scheme for an underwater channel which are able to provide transmit diversity by means of space-frequency codes (Chap. $4)$. 


\subsection{MIMO channel model}

We will consider a MIMO system with $M_{T}$ transmitters and $M_{R}$ receivers. The channel impulse response between the $t$-th transmitter $\left(t=1 \ldots M_{T}\right)$ and the $r$-th receiver $\left(r=1 \ldots M_{R}\right)$ will be denoted by $h^{t, r}(\tau, t)$. The MIMO channel is given by the following $M_{R} \times M_{T}$ matrix

$$
\mathbf{H}(\tau, t)=\left[\begin{array}{cccc}
h^{1,1}(\tau, t) & h^{2,1}(\tau, t) & \cdots & h^{M_{T}, 1}(\tau, t) \\
h^{1,2}(\tau, t) & h^{2,2}(\tau, t) & \cdots & h^{M_{T}, 2}(\tau, t) \\
\vdots & \vdots & \ddots & \vdots \\
h^{1, M_{R}}(\tau, t) & h^{2, M_{R}}(\tau, t) & \cdots & h^{M_{T}, M_{R}}(\tau, t)
\end{array}\right]
$$

This notation provides a complete definition of the channel in time domain between multiple transmitters and receivers. However, to further simplify the notation, and given that our system is based on the OFDM modulation, it is reasonable to assume that each subcarrier is an independent MIMO system over a non-selective channel. The channel transfer function observed on the $k$-th carrier during the $n$-th OFDM block will be denoted by

$$
\mathbf{H}_{k}(n)=\left[\begin{array}{cccc}
H_{k}^{1,1}(n) & H_{k}^{2,1}(n) & \cdots & H_{k}^{M_{T}, 1}(n) \\
H_{k}^{1,2}(n) & H_{k}^{2,2}(n) & \cdots & H_{k}^{M_{T}, 2}(n) \\
\vdots & \vdots & \ddots & \vdots \\
H_{k}^{1, M_{R}}(n) & H_{k}^{2, M_{R}}(n) & \cdots & H_{k}^{M_{T}, M_{R}}(n)
\end{array}\right]
$$

\subsection{Diversity and multiplexing}

The wireless link performance can be greatly improved by using a multiple-input multiple-output channel, both in terms of reliability and data rate. In other words, the channel provides two types of performance gains. In this section, these two types of gains will be studied separately. The trade-off relation between them will be introduced in Sec.3.3.

\subsubsection{Diversity gain}

In MIMO communications, multiple transmitters and/or receivers can be used to improve the communication reliability, i.e. to provide spatial diversity. The main idea is to supply the receiver with many independently faded replicas of the same signal so that the probability that all the signal components fade simultaneously is greatly reduced. Figure 3.1 shows different configurations for spatial diversity.

Let us present an example to show the theoretical benefits of spatial diversity. In this example we will consider the error probability at high SNR of an uncoded binary PSK signal over a single antenna fading channel. It is well known [15] that the error probability averaged over the fading gain and additive noise is

$$
P_{e_{(1 \times 1)}}(\mathrm{SNR}) \approx \frac{1}{4} \mathrm{SNR}^{-1} \propto \mathrm{SNR}^{-1}
$$




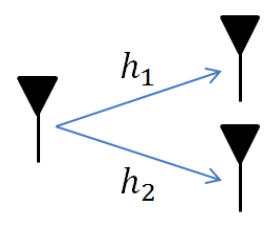

(a)

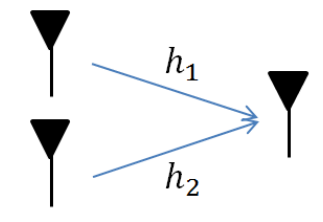

(b)

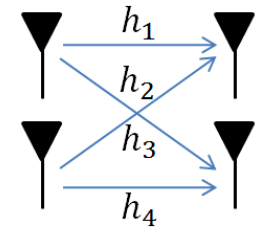

(c)

Figure 3.1: (a) Receive diversity. (b) Transmit diversity. (c) Both transmit and receive diversity.

In high SNR regime, this error probability is mainly attributed to the channel faded components, hence

$$
P_{e_{(1 \times 1)}}(\mathrm{SNR}) \approx \operatorname{Pr}\left\{h^{1,1} \text { in fade }\right\} \propto \mathrm{SNR}^{-1}
$$

In contrast, when the same signal is transmitted to a system equipped with two receivers, the probability of error is

$$
P_{e_{(1 \times 2)}}(\mathrm{SNR}) \approx \operatorname{Pr}\left\{h^{1,1} \text { in fade, } h^{1,2} \text { in fade }\right\}
$$

Since these receivers are supposed to be spaced several wavelengths, the fading is assumed to be independent and we have

$$
P_{e_{(1 \times 2)}}(\mathrm{SNR}) \approx \operatorname{Pr}\left\{h^{1,1} \text { in fade }\right\} \cdot \operatorname{Pr}\left\{h^{1,2} \text { in fade }\right\} \propto \mathrm{SNR}^{-2}
$$

The same result can be achieved with two transmitters by using, for instance, the repetition scheme. This scheme consists of transmitting the same information once from each transmitter in non-overlapped time slots

$$
\mathbf{X}=\operatorname{space}\left[\begin{array}{cc}
x_{1} & 0 \\
0 & x_{1}
\end{array}\right]
$$

so that each receiver has $M_{T}$ replicas of the signal collected over $M_{T}$ channel uses. This case is equivalent to receive diversity, provided that the receiver records two independent realizations of the channel fading, therefore the same error probability applies for this case.

As we have observed, the employment of either two transmitters or two receivers yields an error probability that decreases with the SNR, faster than $\mathrm{SNR}^{-2}$. Consequently, at high SNR regions, the error probability is relatively much smaller when spatial diversity is employed. The same trend is maintained with more than two elements. In general, each transmitter-receiver pair provides an independent realization of the fading channel, so the error probability for an arbitrary number of $M_{T}, M_{R}$ is

$$
P_{e_{\left(M_{T} \times M_{R}\right)}} \propto \mathrm{SNR}^{-M_{T} M_{R}}
$$

where the exponent dictates the performance gain at high SNR. This exponent is called the diversity gain $\left(d_{G}\right)$ and its upper bound is given by the total number of transmitter-receiver 
combinations. Ideally, the maximum achievable diversity gain for a fixed target rate $R$ in a $M_{T} \times M_{R}$ MIMO system is

$$
d_{G}^{*}=M_{T} M_{R}
$$

\subsubsection{Multiplexing gain}

Besides providing diversity to improve reliability, MIMO channels can support higher data rates as compared to single channel systems. To show the benefits of spatial multiplexing, we take a closer look at the channel capacity and derive the best way to maximize it.

Consider a $M_{R} \times M_{T}$ narrowband time-invariant MIMO channel, denoted by the matrix $\mathbf{H}$ (3.2). The transmission over this channel is denoted by

$$
\mathbf{y}=\mathbf{H x}+\mathbf{z}
$$

where $\mathbf{x} \in \mathbb{C}^{M_{T}}, \mathbf{y} \in \mathbb{C}^{M_{R}}$ and $\mathbf{z} \sim \mathcal{N}\left(0, N_{0} \mathbf{I}_{M_{R}}\right)$ are the transmitted signal, the received signal and additive white Gaussian noise, respectively. The channel matrix $\mathbf{H}$ is deterministic and assumed to be known to both the transmitter and the receiver. The communication channel is a vector Gaussian channel, whose capacity in single-transmitter single-receiver scenarios is given by

$$
C \leq \log _{2}(1+\mathrm{SNR}) \quad[\mathrm{bps} / \mathrm{Hz}]
$$

and equivalently for MIMO scenarios

$$
C \leq \log _{2}\left(\left|\mathbf{I}_{M_{R}}+\frac{1}{N_{0}} \mathbf{H R}_{\mathbf{x}} \mathbf{H}^{H}\right|\right)
$$

where $\mathbf{R}_{\mathbf{x}}=\mathbf{x} \mathbf{x}^{H}$ is the covariance matrix of the transmitted signal, which is a function of the power allocation, i.e. $\mathbf{R}_{\mathbf{x}}=\operatorname{diag}\left(P_{1}, P_{2}, \ldots, P_{M_{T}}\right)$.

The capacity can be computed by decomposing the vector channel into a set of parallel, independent scalar Gaussian sub-channels. To do so, we take advantage of the singular value decomposition (SVD), which decomposes any matrix (linear transformation) into a product of three other matrices. The resulting matrices represent three basic operations: rotation, scaling and rotation, respectively. Therefore, the channel matrix can be decomposed in the following manner

$$
\mathbf{H}=\mathbf{U} \boldsymbol{\Lambda} \mathbf{V}^{H}
$$

where $\mathbf{U} \in \mathbb{C}^{M_{R} \times M_{R}}$ and $\mathbf{V} \in \mathbb{C}^{M_{T} \times M_{T}}$ are unitary matrices, i.e. $\mathbf{U}^{H} \mathbf{U}=\mathbf{I}_{M_{R}}$ and $\mathbf{V}^{H} \mathbf{V}=\mathbf{I}_{M_{T}}$. The matrix $\boldsymbol{\Lambda} \in \mathbb{C}^{M_{R} \times M_{T}}$ is a rectangular matrix whose diagonal elements are the non-negative real eigenvalues of $\mathbf{H}$, and the off-diagonal elements are zero. The set of eigenvalues that compose the diagonal are ordered by magnitude, i.e. $\lambda_{1} \geq \lambda_{2} \geq \ldots \geq \lambda_{n_{\text {min }}}$ with

$$
n_{\min }=\min \left(M_{T}, M_{R}\right)
$$


In order to take advantage of the channel knowledge, both the transmitter and the receiver apply a linear transformation to the transmitted and received signals, such that

$$
\begin{aligned}
\mathbf{x}^{\prime} & =\mathbf{V} \mathbf{x} \\
\mathbf{y}^{\prime} & =\mathbf{U}^{H} \mathbf{y}
\end{aligned}
$$

This alters the channel capacity in the following way

$$
C \leq \log _{2}\left(\left|\mathbf{I}_{M_{R}}+\frac{1}{N_{0}} \mathbf{H V R}_{\mathbf{x}} \mathbf{V}^{H} \mathbf{H}^{H}\right|\right)
$$

Taking into account that two arbitrary matrices $\mathbf{A} \in \mathbb{C}^{m \times n}$ and $\mathbf{B} \in \mathbb{C}^{n \times m}$ satisfy $\left|\mathbf{I}_{m}+\mathbf{A B}\right|=$ $\left|\mathbf{I}_{n}+\mathbf{B A}\right|$, the capacity can be rewritten and simplified

$$
\begin{aligned}
C & \leq \log _{2}\left(\left|\mathbf{I}_{M_{T}}+\frac{1}{N_{0}} \mathbf{R}_{\mathbf{x}} \mathbf{V}^{H} \mathbf{H}^{H} \mathbf{H} \mathbf{V}\right|\right)= \\
& =\log _{2}\left(\left|\mathbf{I}_{M_{T}}+\frac{1}{N_{0}} \mathbf{R}_{\mathbf{x}} \mathbf{V}^{H} \mathbf{V} \boldsymbol{\Lambda}^{H} \mathbf{U}^{H} \mathbf{U} \boldsymbol{\Lambda} \mathbf{V}^{H} \mathbf{V}\right|\right)= \\
& =\log _{2}\left(\left|\mathbf{I}_{M_{T}}+\mathbf{R}_{\mathbf{x}} \frac{\boldsymbol{\Lambda}^{H} \boldsymbol{\Lambda}}{N_{0}}\right|\right)=\log _{2}\left(\prod_{i=1}^{n_{\min }} 1+\frac{P_{i} \lambda_{i}^{2}}{N_{0}}\right)
\end{aligned}
$$

which, effectively, is the sum of the capacities of each independent channel

$$
C \leq \sum_{i=1}^{n_{\min }} \log _{2}\left(1+\frac{P_{i} \lambda_{i}^{2}}{N_{0}}\right)
$$

This result shows that the capacity of the MIMO channel is the sum of capacities of each singleinput single-output (SISO) channel. Such MIMO system is schematically represented in Fig.3.2. Since the transmitter has access to different spatial channels, it can allocate different powers $\left(P_{1}, P_{2}, \ldots P_{n_{m i n}}\right)$ to maximize the mutual information.

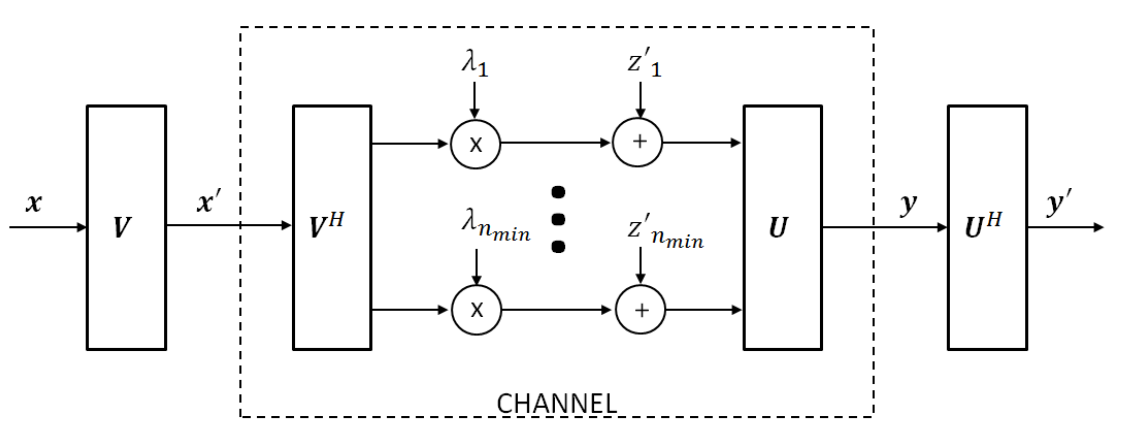

Figure 3.2: MIMO channel converted into a parallel channel through SVD. 


\section{Water-filling algorithm}

In the last section we showed that the MIMO channel is equivalent, in terms of capacity, to the sum of each individual parallel SISO channel. Additionally, the transmitter may allocate a different power to each of these sub-channels. Hence, a maximization problem arises. To avoid the trivial solution $P_{i} \rightarrow \infty, P_{i}$ needs to be constrained to a maximum available power at the transmitter side. The mutual information maximization problem now becomes

$\max _{P_{i}} \sum_{i=1}^{n_{\min }} \log _{2}\left(1+\frac{P_{i} \lambda_{i}^{2}}{N_{0}}\right)$

s.t.

$\sum_{i=1}^{n_{\min }} P_{i}=P_{\max }$

Since the function to be maximized is concave in the variables $P_{i}$, the problem can be solved using Lagrange methods

$$
\frac{\partial}{\partial P_{i}} \sum_{i=1}^{n_{\min }} \log _{2}\left(1+\frac{\lambda_{i}^{2} P_{i}}{N_{0}}\right)-\nu\left(\sum_{i=1}^{n_{\min }} P_{i}-P_{\max }\right)=\frac{\ln 2 \frac{\lambda_{i}^{2}}{N_{0}}}{1+\frac{\lambda_{i}^{2} P_{i}}{N_{0}}}-\nu=0
$$

Isolating $P_{i}$ we obtain the optimal power allocation

$$
P_{i}^{*}=\mu-\frac{N_{0}}{\lambda_{i}^{2}} \quad i=1, \ldots, n_{\min }
$$

where $\mu=\frac{\ln 2}{\nu}$. We now proceed to calculate the constant $\mu$. Bearing in mind that $\sum_{i} P_{i}^{*}=P_{\max }$, we obtain

$$
\mu=\frac{1}{n_{\min }}\left[P_{\max }+N_{0} \sum_{i=1}^{n_{\min }} \frac{1}{\lambda_{i}^{2}}\right]
$$

At this point we have a complete set of equations (3.22), (3.21) that allows us to compute the optimal power allocation. However, the obtained powers may eventually be set to negative values in order to favor other modes with bigger channel gain. If the power allocated to the channel with the lowest gain is negative, i.e. $P_{n_{m i n}}^{*}<0$, we discard this channel mode by setting $P_{n_{m i n}}^{*}=0$. The optimal values for $P_{i}$ are calculated and discarded iteratively until the power allocated to each spatial sub-channel is non-negative.

To give it a pictorially view, the variable $\mu$ represents a certain water level, and $\lambda_{i}^{2} / N_{0}$ represents the water depth of each sub-channel. When the depth corresponding to the smallest eigenvalue exceeds the water surface, i.e. is negative, it is discarded and the water level is recalculated. Note that when (3.22) is recomputed without the last eigenvalue the water level varies. The algorithm continues in this fashion until there is enough water to have all modes submerged. 


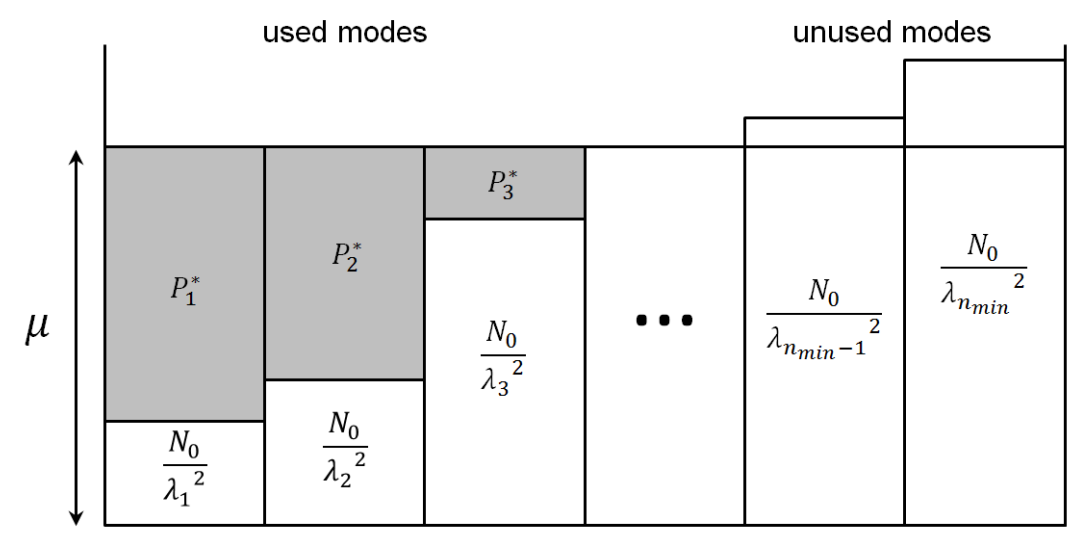

Figure 3.3: Schematic of the water-filling algorithm.

Figure 3.3 summarizes the outcome of the water-filling algorithm. The algorithm is detailed in Alg.1.

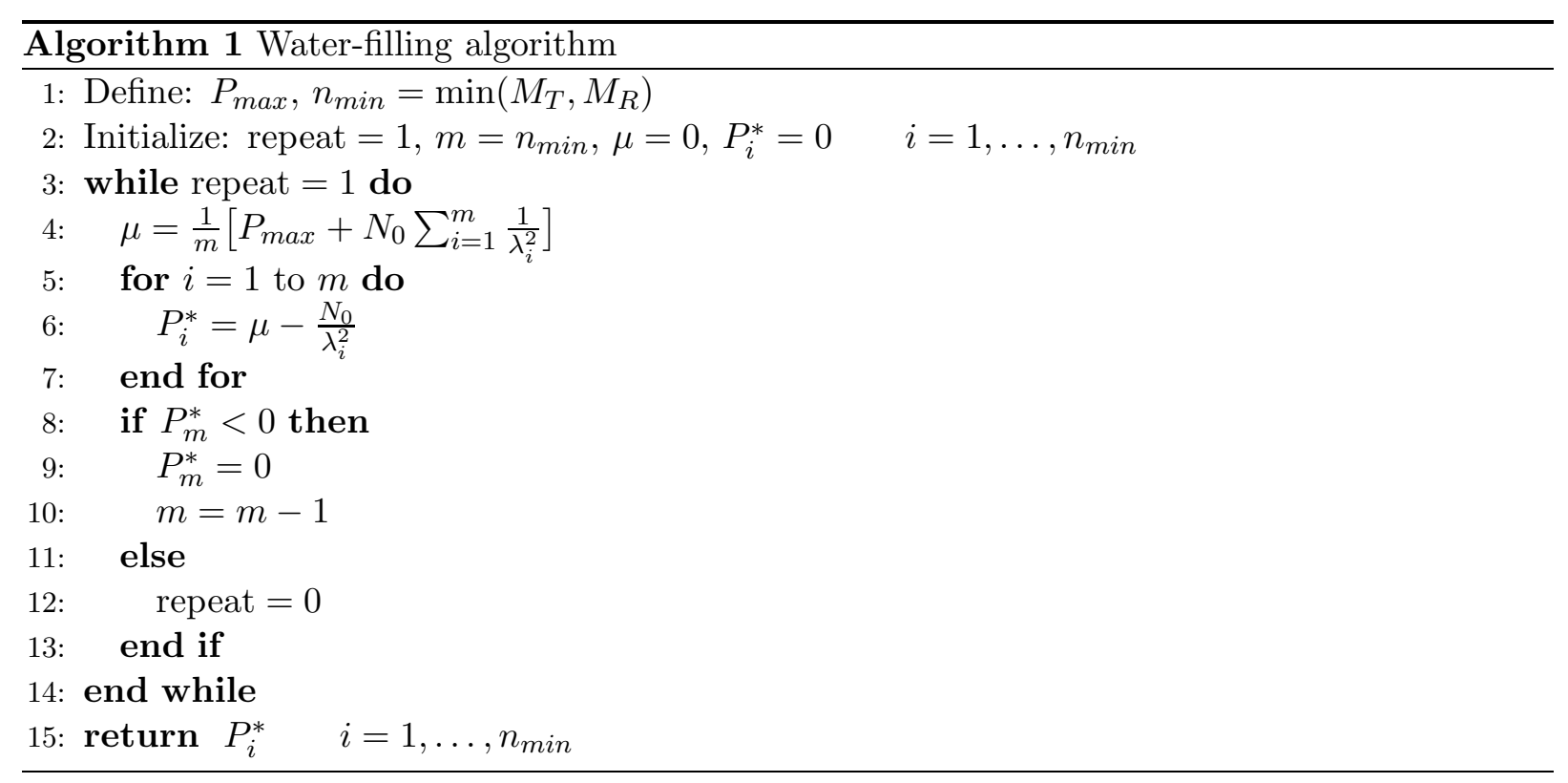

\subsection{Diversity-multiplexing tradeoff}

In the above section we have analysed the types of gains yielded by MIMO channels. A key measure of the performance capability of a MIMO channel is the maximum diversity gain that can be extracted from it. This kind of gain is most likely to be achieved in slow fading scenarios. For example, the maximum gain in a slow i.i.d. Rayleigh faded channel with $M_{T}$ transmitters and $M_{R}$ receivers is $M_{T} \cdot M_{R}$, i.e., for a fixed target rate $R$ the outage probability $P_{\text {out }}(R)$ scales like $\mathrm{SNR}^{-M_{T} \cdot M_{R}}$ when $\mathrm{SNR} \rightarrow \infty$.

On the other hand, fast fading scenarios are attractive because they provide additional degrees 
of freedom and increase the effectiveness of spatial multiplexing. In this case, the capacity of an i.i.d. Rayleigh fading channel scales like $n_{\min } \log _{2}(\mathrm{SNR})$, where $n_{\min }=\min \left(M_{T}, M_{R}\right)$ is the number of available degrees of freedom in the channel.

One may notice that in order to achieve the maximum diversity gain, the rate $R$ at which the system communicates is relatively small as compared to the maximum achievable capacity in fast fading environments. Thus, one is actually sacrificing all the spatial multiplexing benefit of the MIMO channel to maximize the reliability. To take advantage of some of that benefit one can communicate at a rate $R=r \log _{2}(\mathrm{SNR})$, which is a fraction of the maximum capacity, while keeping some diversity gain. Hence, it makes sense to formulate the following diversity-multiplexing tradeoff for a slow fading channel: a diversity gain $d_{G}(r)$ is achieved at a multiplexing gain $r$ if

$$
R=r \log _{2}(\mathrm{SNR})
$$

and the outage probability for a target rate $R$, defined as $P_{\text {out }}(R)=\operatorname{Pr}\{C<R\}$ is

$$
P_{\text {out }}(R) \approx \mathrm{SNR}^{-d_{G}(r)}
$$

consequently,

$$
\lim _{\mathrm{SNR} \rightarrow \infty} \frac{\log P_{\text {out }}\left(r \log _{2}(\mathrm{SNR})\right)}{\log (\mathrm{SNR})}=-d_{G}(r)
$$

The curve $d_{G}(r)$ characterizes the slow-fading performance bound of the channel.

To illustrate the tradeoff curve, consider a PAM modulation over a scalar slow fading Rayleigh channel. The average error probability of this modulation is determined by the minimum distance between the constellation points. At high SNR, the error probability [17] is approximately

$$
P_{e} \approx \frac{1}{2}\left(1-\sqrt{\frac{D_{\min }^{2}}{4+D_{\min }^{2}}}\right) \approx \frac{1}{D_{\min }^{2}}
$$

Hence, since the constellation ranges approximately from $-\sqrt{\mathrm{SNR}}$ to $\sqrt{\mathrm{SNR}}$, and there are $2^{R}$ different symbols, where the minimum distance is approximately

$$
D_{\min }=\frac{\sqrt{\mathrm{SNR}}}{2^{R}}
$$

Now we set the desired data rate $R=r \log _{2}(\mathrm{SNR})$ and we obtain

$$
P_{e} \approx \frac{1}{D_{\min }^{2}}=\frac{2^{2 R}}{\mathrm{SNR}}=\frac{2^{2 r \log _{2}(\mathrm{SNR})}}{\mathrm{SNR}}=\frac{1}{\mathrm{SNR}^{1-2 r}}
$$

thus, this yields a diversity-multiplexing tradeoff of

$$
d_{G_{P A M}}(r)=1-2 r \quad r \in[0,1 / 2]
$$


Analogously, for a QAM modulation, the symbols are distributed in two dimensions, so now the minimum distance is

$$
D_{\min }=\frac{\sqrt{\mathrm{SNR}}}{2^{R / 2}}
$$

and the error probability

$$
P_{e} \approx \frac{2^{R}}{\mathrm{SNR}}=\frac{1}{\mathrm{SNR}^{1-r}}
$$

yielding a tradeoff of

$$
d_{G_{Q A M}}(r)=1-r \quad r \in[0,1]
$$

Comparing both schemes, the endpoint value $d_{G}^{*}=d_{G}(0)$ is the exponent that describes how fast the error probability decreases with the SNR, i.e. the diversity gain achieved by the scheme. On the other hand, the value $r_{\max }$ for which $d_{G}\left(r_{\max }\right)=0$ is the number of degrees of freedom that are exploited by the scheme. The diversity gain is 1 in both schemes and the degrees of freedom used are 1/2 and 1 for PAM and QAM, respectively. The tradeoff comparison is shown in Fig.3.4. Other scheme comparisons can be found in [17] for the 2x2 MIMO channel (Fig.3.5).

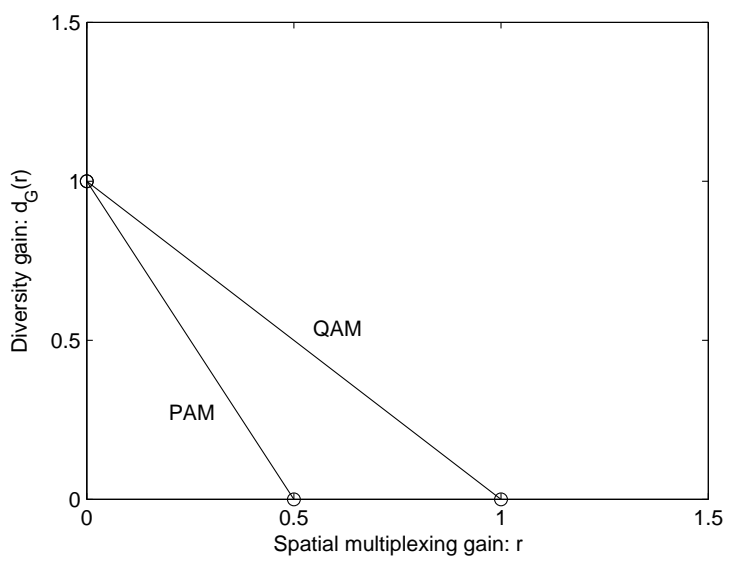

Figure 3.4: Tradeoff for PAM and QAM.

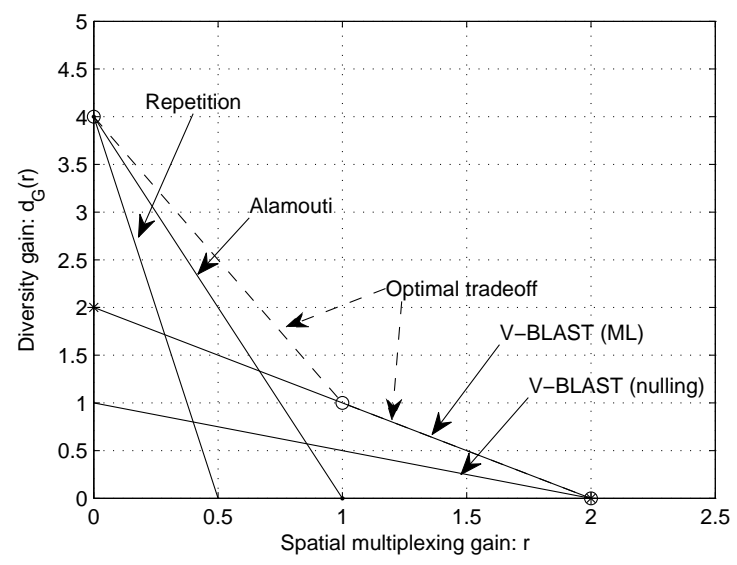

Figure 3.5: Tradeoff for 2x2 MIMO schemes.

\section{Optimal tradeoff}

Once we have compared the PAM-QAM schemes, we would like to know what is the optimal diversity-multiplexing tradeoff of the scalar Rayleigh channel itself. We derive the optimal tradeoff from the outage probability of the channel at a fixed data rate $R=r \log _{2}(\mathrm{SNR})$

$$
P_{\text {out }}=\operatorname{Pr}\left\{\log _{2}\left(1+|h|^{2} \mathrm{SNR}\right)<r \log _{2}(\mathrm{SNR})\right\}=\operatorname{Pr}\left\{|h|^{2}<\frac{\mathrm{SNR}^{r}-1}{\mathrm{SNR}}\right\}
$$

For Rayleigh fading, $\operatorname{Pr}\left\{|h|^{2}<\epsilon\right\} \approx \epsilon$ for small $\epsilon$, thus

$$
P_{\text {out }} \approx \frac{\mathrm{SNR}^{r}-1}{\mathrm{SNR}} \approx \frac{1}{\mathrm{SNR}^{1-r}}
$$


hence the optimal tradeoff for a scalar Rayleigh channel is

$$
d_{G}^{*}(r)=1-r \quad r \in[0,1]
$$

which was achieved by the QAM scheme.

In general, for a $M_{T} \times M_{R}$ i.i.d. Rayleigh channel, the diversity-multiplexing tradeoff is the piecewise linear curve joining the points

$$
\left(r,\left(M_{T}-r\right) \cdot\left(M_{R}-r\right)\right), \quad r=1, \ldots, n_{\text {min }}
$$

so, neither space-time scheme can achieve a diversity-multiplexing tradeoff over the boundary represented in Fig.3.6.

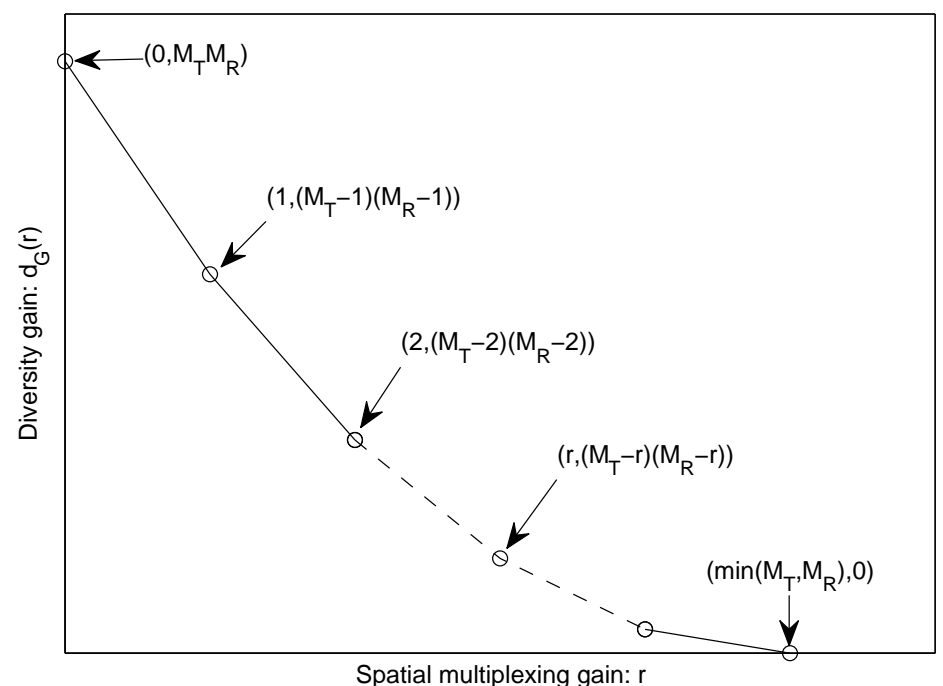

Figure 3.6: Optimal diversity-multiplexing tradeoff curve for a $M_{T} \times M_{R}$ MIMO system.

\subsection{Transmit diversity}

\subsubsection{Transmit diversity with channel knowledge}

In this section we will review the benefits of transmit diversity when the transmitter has perfect knowledge of the channel. Let us assume that our system has $M_{T}$ transmitters and $M_{R}$ receivers. Let $\mathbf{b}$ be a beamforming vector of size $M_{T} \times 1$, and $\mathbf{A}$ the decoding matrix. The MIMO channel is denoted by the matrix $\mathbf{H}$. With the above notation, we denote the transmitted signal $\mathbf{x}(n)$ 
$\left(\mathbf{x} \in \mathbb{C}^{M_{T} \times 1}\right)$ and received signal $\mathbf{y}(n)\left(\mathbf{y} \in \mathbb{C}^{M_{R} \times 1}\right)$ as

$$
\begin{aligned}
& \mathbf{x}(n)=\mathbf{b} \cdot d(n) \\
& \mathbf{y}(n)=\mathbf{H b} \cdot d(n)+\mathbf{z}(n)
\end{aligned}
$$

where $d(n)$ is a scalar symbol.

Since only one symbol is being transmitted, the receiver performs the trace operation after applying the decoding matrix to further simplify the decoding process. So, at the output of the decoder we have

$$
\operatorname{tr}\left(\mathbf{A}^{H} \mathbf{y}(n)\right)=\operatorname{tr}\left(\mathbf{A}^{H} \mathbf{H} \mathbf{b}\right) \cdot d(n)+\operatorname{tr}\left(\mathbf{A}^{H} \mathbf{z}(n)\right)
$$

hence, the corresponding SNR is

$$
\mathrm{SNR}=\frac{\operatorname{tr}^{2}\left(\mathbf{A}^{H} \mathbf{H} \mathbf{b}\right)}{\operatorname{tr}\left(\mathbf{A}^{H} \mathbf{R}_{0} \mathbf{A}\right)}
$$

where $\mathbf{R}_{0}=\mathbf{z z}^{H}$. Setting $\mathbf{C}=\mathbf{R}_{0}^{\frac{1}{2}} \mathbf{A}$ and $\mathbf{D}=\mathbf{R}_{0}^{-\frac{1}{2}} \mathbf{H b}$, the following inequality (see appendix A.1) will be used to maximize the SNR

$$
\operatorname{tr}^{2}\left(\mathbf{C}^{H} \mathbf{D}\right) \leq \operatorname{tr}\left(\mathbf{C} \mathbf{C}^{H}\right) \cdot \operatorname{tr}\left(\mathbf{D} \mathbf{D}^{H}\right) \quad \text { equal if } \mathbf{C} \propto \mathbf{D}
$$

The upper boundary for the SNR is then

$$
\mathrm{SNR}_{\max }=\mathbf{b}^{H} \mathbf{H}^{H} \mathbf{R}_{0}^{-1} \mathbf{H b}
$$

The optimal precoder and decoder arise when

- the inequality verifies, i.e. $\mathbf{C}=\mathbf{D}$. Then we have

$$
\mathbf{A}_{\text {opt }}=\mathbf{R}_{0}^{-1} \mathbf{H b}
$$

- the precoder $\mathbf{b}$ maximizes $\mathrm{SNR}_{\max }$. Consequently, $\mathbf{b}$ is the maximum eigenvector of $\mathbf{R}_{H}=$ $\mathbf{H}^{H} \mathbf{R}_{0}^{-1} \mathbf{H}$ multiplied by $\sqrt{E_{T}}$, where $E_{T}$ is the transmitted energy. The resulting SNR is

$$
\mathrm{SNR}_{\text {max }}=E_{T} \lambda_{\text {max }}\left(\mathbf{R}_{H}\right)
$$

In conclusion, to achieve the maximum SNR, the transmitter takes advantage of the channel eigenvalues and assigns all the available power to the best eigenmode.

\subsubsection{Transmit diversity over an unknown channel}

Generally, in wireless communications, it is usual to have only channel state information at the receiver (CSIR), i.e. the channel is not known to the transmitter. In this case the channel matrix 
cannot be used in the precoding block as the transmitter does not have knowledge of the best eigenmode. In this section we will derive an optimal precoding scheme, which yields a transmit diversity gain equal to MRC when the channel is only known to the receiver.

Let us focus on a MIMO scenario with $M_{T}$ transmitters and $M_{R}$ receivers. The transmission is performed using a rectangular matrix $\mathbf{B}=\left[\begin{array}{lll}\mathbf{b}_{1} & \ldots & \mathbf{b}_{L}\end{array}\right] \in \mathbb{C}^{M_{t} \times L}$, whose dimensions are space (rows) and time (columns) and $L$ is the number of channel uses employed to transmit a block code $^{1}$. The transmitted signal vector $\mathbf{x}_{l} \in \mathbb{C}^{M_{T}}$ during the $l$-th channel use can be written as

$$
\mathbf{x}_{l}=\mathbf{b}_{l} d(n) \quad l=1, \ldots L
$$

where $d(n)$ is now a real-valued scalar symbol.

The channel transfer function is denoted by $\mathbf{H} \in \mathbb{C}^{M_{R} \times M_{T}}$, as described in (3.2). The channel coherence time is supposed to be long enough for $\mathbf{H}$ to remain constant at least during the $L$ channel uses. Taking into account the mentioned assumptions, we denote the signal arriving at the receiver array during the $l$-th channel use as

$$
\mathbf{y}_{l}=\mathbf{H b}_{l} d(n)+\mathbf{z}_{l}(n)
$$

where $\mathbf{z}_{l}(n)$ represents zero-mean additive noise. Equation (3.45) can be expressed in a more compact form by packing each individually transmitted vector into a single matrix

$$
\mathbf{Y}=\left[\begin{array}{lll}
\mathbf{y}_{1} & \ldots & \mathbf{y}_{L}
\end{array}\right]=\mathbf{H B} d(n)+\mathbf{Z}
$$

The received signal is processed with a receiver block $\mathbf{A} \in \mathbb{C}^{M_{R} \times L}$, which will yield the maximum SNR

$$
\mathbf{Y}^{\prime}=\mathbf{A}^{H} \mathbf{Y}=\mathbf{A}^{H} \mathbf{H B} d(n)+\mathbf{A}^{H} \mathbf{Z}
$$

Bearing in mind that the transmitted symbols are scalar values, the trace operation is performed after the linear transformation, so as to simplify the decoding process

$$
y^{\prime \prime}=\operatorname{tr}\left(\mathbf{Y}^{\prime}\right)=\operatorname{tr}\left(\mathbf{A}^{H} \mathbf{H B} d(n)\right)+\operatorname{tr}\left(\mathbf{A}^{H} \mathbf{Z}\right)
$$

The final received signal $y^{\prime \prime}$ consists of the sum of two scalar values: the signal contribution $s(n)=\operatorname{tr}\left(\mathbf{A}^{H} \mathbf{H B}\right) d(n)$, and the noise.

In order to design the optimal receiver, several more definitions are necessary. The received signal energy is given by

$$
E_{r}=E\left\{\left\|s_{r}(n)\right\|^{2}\right\}=\operatorname{tr}^{2}\left(\mathbf{A}^{H} \mathbf{H B}\right) E_{s}
$$

where $E_{s}$ is the mean symbol energy. An expression for the SNR is obtained by dividing (3.48)

\footnotetext{
${ }^{1}$ Note that more than one symbol can be multiplexed simultaneously.
} 
by the noise energy. Considering $\mathbf{R}_{0}=E\left\{\mathbf{Z}^{H} \mathbf{Z}\right\}$, we have

$$
\mathrm{SNR}=E_{s} \frac{\operatorname{tr}^{2}\left(\mathbf{A}^{H} \mathbf{H B}\right)}{\operatorname{tr}\left(\mathbf{A}^{H} \mathbf{R}_{0} \mathbf{A}\right)}
$$

\section{Optimal receiver by SNR maximization}

In this section, the optimal receiver will be derived from the SNR maximization problem. Using the trace inequalities given by (3.40), it is possible to obtain a upper bound for the SNR. Thereafter, one can apply the required constraints for the inequalities to be equal, and derive the optimal receiver. We first focus on the maximization process, in which the goal is to maximize (3.49). This can be easily done using the following inequality (see appendix A.1)

$$
\operatorname{tr}^{2}\left(\mathbf{C}^{H} \mathbf{D}\right) \leq \operatorname{tr}\left(\mathbf{C} \mathbf{C}^{H}\right) \cdot \operatorname{tr}\left(\mathbf{D} \mathbf{D}^{H}\right)
$$

equivalently we obtain

$$
\frac{\operatorname{tr}^{2}\left(\mathbf{C}^{H} \mathbf{D}\right)}{\operatorname{tr}\left(\mathbf{C} \mathbf{C}^{H}\right)} \leq \operatorname{tr}\left(\mathbf{D} \mathbf{D}^{H}\right)
$$

where the equality verifies if $\mathbf{C}=\mathbf{D}$. It is possible to take advantage of the similarity between expressions to rewrite (3.49) with the following identities

$$
\left.\begin{array}{l}
\mathbf{C}^{H} \mathbf{D}=\mathbf{A}^{H} \mathbf{H B} \\
\mathbf{C}^{H} \mathbf{C}=\mathbf{A}^{H} \mathbf{R}_{0} \mathbf{A}
\end{array}\right\} \quad \mathbf{C}=\mathbf{R}_{0}^{\frac{1}{2}} \mathbf{A} \quad \mathbf{D}=\mathbf{R}_{0}^{-\frac{1}{2}} \mathbf{H B}
$$

substituting into (3.50), an upper bound for the SNR arises

$$
\mathrm{SNR} \leq \operatorname{tr}\left(\mathbf{B}^{H} \mathbf{H}^{H} \mathbf{R}_{0}^{-1} \mathbf{H B}\right) E_{s}
$$

To achieve the maximum value for SNR, the equality $\mathbf{C}=\mathbf{D}$ has to be satisfied, which means that

$$
\mathbf{R}_{0}^{\frac{1}{2}} \mathbf{A}=\mathbf{R}_{0}^{-\frac{1}{2}} \mathbf{H B}
$$

resulting in

$$
\mathbf{A}_{\text {opt }}=\mathbf{R}_{0}^{-1} \mathbf{H B}
$$

\section{Optimal transmission code}

Once the optimal receiver has been determined, we proceed to design the transmission matrix $\mathbf{B}$. This matrix has some design conditions that are worth considering:

- The transmission matrix is designed at the transmitter side. Therefore, the matrix $\mathbf{H}$ cannot be used in the design of $\mathbf{B}$.

- The transmission matrix is also present in the optimal receiver (3.52). In consequence, both the transmitter and the receiver have a-priori knowledge of $\mathbf{B}$. 
To simplify the notation of this section, the channel-noise product present in (3.51) will be named $\mathbf{R}_{\mathbf{H}} \equiv \mathbf{H}^{H} \mathbf{R}_{0}^{-1} \mathbf{H}$.

The design of $\mathbf{B}$ can be viewed as a game between the channel and the transmitter. The game opponents are the channel and the precoder, whose goals are to minimize and maximize the SNR, respectively. Let us define the game rules:

\begin{tabular}{|l|l|ll|}
\hline Player & Objective & Rules & \\
\hline \hline Channel $\mathbf{R}_{\mathbf{H}}$ & Minimize SNR & $\operatorname{tr}\left(\mathbf{R}_{\mathbf{H}}\right) \geq \rho$ & Channel eigenvalues $>0$ \\
\hline Precoder $\mathbf{B}$ & Maximize SNR & $\operatorname{tr}\left(\mathbf{B B}^{H}\right) \leq \frac{E_{t}}{E_{s}}$ & Limited power \\
\hline
\end{tabular}

In the table, $E_{t}$ is the total transmitted energy. The rules are designed to be fair, in a way that the first player cannot beat out the second in a single move and vice versa.

The channel plays first, trying to minimize SNR. The following inequality is valid for any arbitrary couple of hermitian matrices (see appendix A.1)

$$
\operatorname{tr}(\mathbf{C D}) \geq \lambda_{\min }(\mathbf{C}) \operatorname{tr}(\mathbf{D})
$$

where $\lambda_{\min }(\cdot)$ represents the minimum eigenvalue. The left side is equivalent to the maximum SNR bound (3.51) when $\mathbf{C}=\mathbf{B B}^{H}$ and $\mathbf{D}=\mathbf{R}_{\mathbf{H}}$. The inequality offers an opportunity to decrease the minimum SNR bound. Hence, the best the channel can do is to decrease its own trace until the minimum allowed value (defined as $\rho$ in the rules). It does so by $\operatorname{setting} \operatorname{tr}\left(\mathbf{R}_{\mathbf{H}}\right)=\rho$ and leaves the game with

$$
\mathrm{SNR}_{\text {max }} \geq \lambda_{\min }\left(\mathbf{B B}^{H}\right) \cdot \rho \cdot E_{s}
$$

Now it's the precoder's turn. The last move done by the channel has decreased the minimum SNR bound, so the precoder's interest is to maximize $\mathbf{B B}^{H}$ in order to achieve the maximum SNR. To solve the maximization problem, we set it out as

$$
\max _{\mathbf{B B}^{H}}\left[\lambda_{\min }\left(\mathbf{B B}^{H}\right) \cdot \rho \cdot E_{s}\right]=\max _{\mathbf{B B}^{H}}\left[\lambda_{\min }\left(\mathbf{B B}^{H}\right)\right]
$$

which leads to the following solution

$$
\mathbf{B B}^{H}=\frac{E_{t}}{E_{s}} \frac{1}{M_{T}} \mathbf{I}_{M_{T}}
$$

This result is worth a brief dimensional analysis. The so-obtained equivalence (3.53) verifies only if $\operatorname{rank}(\mathbf{B})=M_{T}$. Since $\mathbf{B} \in \mathbb{C}^{M_{T} \times L}$, this means that

$$
L \geq M_{T}
$$


i.e., the length of the block code must be at least equal to the number of transmitters. The final result represents a non-full-rate system with rate $\frac{1}{M_{T}} \mathrm{symbol} / \mathrm{pcu}$, and maximum SNR equal to

$$
\mathrm{SNR}_{\text {max }}=\frac{E_{t}}{M_{T}} \operatorname{tr}\left(\mathbf{R}_{\mathbf{H}}\right)=E_{t} \frac{1}{M_{T}} \sum_{i=1}^{M_{T}} \lambda_{i}^{\left(\mathbf{R}_{\mathbf{H}}\right)}=E_{t} \cdot \bar{\lambda}\left(\mathbf{R}_{\mathbf{H}}\right)
$$

\section{Full-rate transmit diversity scheme: The Alamouti code}

The optimal precoder obtained in (3.53) sets a constraint on the correlation, but leaves a degree of freedom to choose any matrix that, when multiplied by its hermitian, satisfies the identity. To achieve a full-rate system, many symbols will be multiplexed simultaneously, while the remaining degrees of freedom will be used to cancel the interference among them.

In this section we study the precoder design for a system with $M_{T}=2$ transmitters and an arbitrary number of receivers. We show that a set of precoding matrices that allow full-rate transmission indeed exists. This transmission scheme is known as the Alamouti Code.

Let us extend the system described above by adding additional branches both at the transmitter and the receiver sides, as shown in Figure 3.7. Now the transmitter multiplexes four symbols,
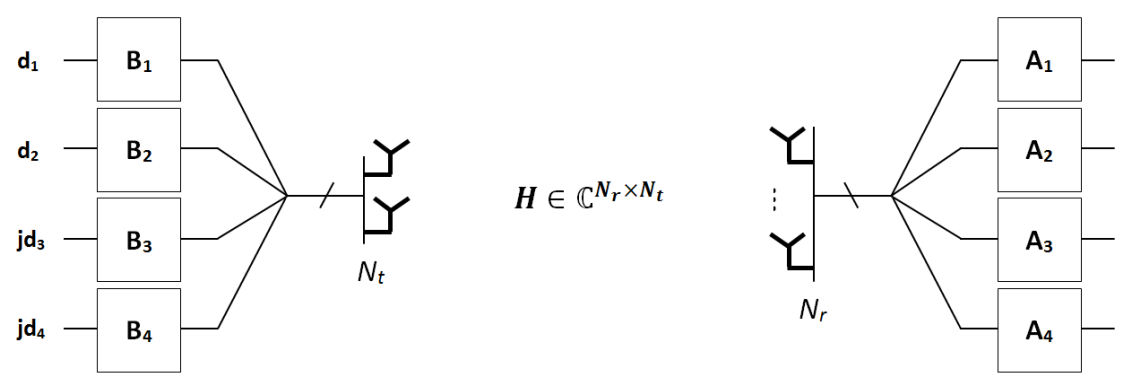

Figure 3.7: Symbol multiplexing scenario

each one with a different precoder. Thereafter, the symbols are added up and transmitted.

The receivers are chosen to be optimal, so the expression for the $i$-th branch is

$$
\mathbf{A}_{i}=\mathbf{R}_{0}^{-1} \mathbf{H B}_{i}
$$

and the received signal is

$$
\mathbf{Y}_{\mathbf{r}}=\sum_{j=1}^{4} \mathbf{H B}_{i} d_{i}+\mathbf{W}
$$

The trace operation is performed after each receiver. Hence, the signal obtained at the $i$-th branch is

$$
y_{i}=\sum_{j=1}^{4} \operatorname{tr}\left(\mathbf{B}_{i}^{H} \mathbf{H}^{H} \mathbf{R}_{0}^{-1} \mathbf{H} \mathbf{B}_{j}\right) d_{j}+\left(\mathbf{B}_{i}^{H} \mathbf{H}^{H} \mathbf{R}_{0}^{-1} \mathbf{W}\right)
$$

Note that ISI appears for indexes $j \neq i$. Thus each precoder $\mathbf{B}_{i}$ has to be designed so as to cancel the interference produced by the others while satisfying (3.53). To do so, one can find a product 
of precoders that results either in pure real or pure imaginary matrices. The ISI elimination is straightforward thereafter, and only requires the use of $\operatorname{Re}\{\cdot\}$ and $\operatorname{Im}\{\cdot\}$ operations

$$
\begin{aligned}
& \mathbf{B}_{i} \mathbf{B}_{i}^{H}=\frac{E_{t}}{E_{s}} \frac{1}{M_{T}} \mathbf{I}_{M_{R}} \\
& \mathbf{B}_{i} \mathbf{B}_{j}^{H}=-\mathbf{B}_{j} \mathbf{B}_{i}^{H} \quad \Rightarrow \quad \operatorname{Re}\left\{\mathbf{B}_{i} \mathbf{B}_{j}^{H}\right\}=0 \quad(i, j)=\{(1,2),(3,4)\} \\
& \mathbf{B}_{k} \mathbf{B}_{l}^{H}=\mathbf{B}_{l} \mathbf{B}_{k}^{H} \quad \Rightarrow \quad \operatorname{Im}\left\{\mathbf{B}_{k} \mathbf{B}_{l}^{H}\right\}=0 \quad(k, l)=\{(1,3),(1,4),(2,3),(2,4)\}
\end{aligned}
$$

When $\mathbf{B}_{i}$ matrices are designed according to the constraints specified in (3.57), the corresponding symbols can be obtained in each branch after a Real/Imag separation stage, as shown in Figure 3.8.

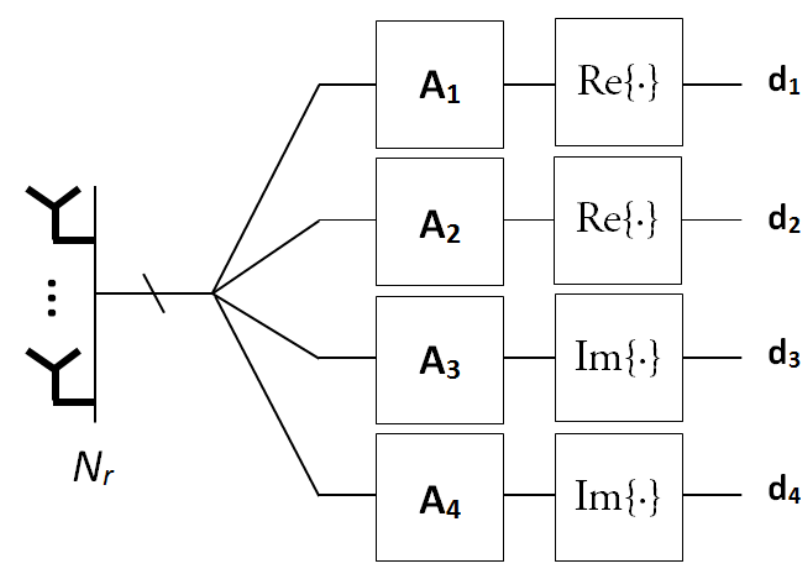

Figure 3.8: Multiplexed symbol receiver with ISI cancellation

One possible solution for $\mathbf{B}_{i}$ that fulfills conditions in (3.57) is

$$
\mathbf{B}_{1}=\left[\begin{array}{ll}
1 & 0 \\
0 & 1
\end{array}\right] \quad \mathbf{B}_{2}=\left[\begin{array}{cc}
0 & -1 \\
1 & 0
\end{array}\right] \quad \mathbf{B}_{3}=\left[\begin{array}{ll}
0 & 1 \\
1 & 0
\end{array}\right] \quad \mathbf{B}_{4}=\left[\begin{array}{cc}
1 & 0 \\
0 & -1
\end{array}\right]
$$

so the transmitted signal is

$$
\mathbf{X}_{t}=\mathbf{B}_{1} d_{1}+\mathbf{B}_{2} d_{2}+\mathrm{j} \mathbf{B}_{3} d_{3}+\mathrm{j} \mathbf{B}_{4} d_{4}=\left[\begin{array}{cc}
d_{1}+\mathrm{j} d_{4} & -d_{2}+\mathrm{j} d_{3} \\
d_{2}+\mathrm{j} d_{3} & d_{1}-\mathrm{j} d_{4}
\end{array}\right]
$$

Considering the symbol pairs as complex symbols $z_{1}=d_{1}+\mathrm{j} d_{4}$ and $z_{2}=d_{2}+\mathrm{j} d_{3}$, the transmission matrix finally results

$$
\mathbf{X}_{t}=\left[\begin{array}{cc}
z_{1} & -z_{2}^{*} \\
z_{2} & z_{1}^{*}
\end{array}\right]
$$

i.e. first antenna transmits $z_{1}$ and then $-z_{2}^{*}$, while simultaneously $z_{2}$ and $z_{1}^{*}$ are transmitted by second antenna.

The use of the Alamouti precoder provides a full-rate system with 1 complex symbol/pcu in 
CSIR. Even though this design can be extended to $M_{T}>2$ antennas to achieve higher diversity gains, there is no feasible solution for the ISI cancellation constraints (3.57) that lead to a fullrate system. However, pretty good rates can still be obtained with higher diversity orders, e.g. 3 symbol $/ 4 \mathrm{pcu}$ when $M_{T}=4$. 


\section{Chapter 4}

\section{SFBC-OFDM system for acoustic channels}

\subsection{System model}

We consider a MIMO system with $M_{T}=2$ transmitters and $M_{R}$ receivers. OFDM is used with $K$ subcarriers, equally spaced within the system bandwidth $B$ at $\Delta f=B / K$. The OFDM symbol duration is $T=1 / \Delta f$, and a guard interval (cyclic prefix) of duration $T_{g}$, sufficient to accommodate the multipath spread $T_{m p}$, is added for the total block duration of $T^{\prime}=T+T_{g}$. The symbols are encoded using the Alamouti SFBC scheme, i.e. if $k$ is the carrier pair index $(k=0 \ldots K / 2-1)$, during the $n$-th OFDM block, the simultaneously transmitted symbols on carriers $2 k$ and $2 k+1$ are, respectively, $d_{2 k}(n), d_{2 k+1}(n)$ from the first transmitter, and $-d_{2 k+1}^{*}(n)$, $d_{2 k}^{*}(n)$ from the second transmitter (3.59).

The channel transfer function observed on the carrier $k^{\prime}$ between transmitter $t$ and receiver $r$ during the $n$-th OFDM block is denoted by $H_{k^{\prime}}^{t, r}(n), k^{\prime}=0 \ldots K-1$. The received signal, corresponding to the $k$-th coded carrier pair and the $r$-th receiving element after OFDM-FFT demodulation, is given by

$$
\mathbf{y}_{2 k}^{r A}(n)=\underbrace{\left[\begin{array}{cc}
H_{2 k}^{1, r}(n) & H_{2 k}^{2, r}(n) \\
-H_{2 k+1}^{2, r *}(n) & H_{2 k+1}^{1, r *}(n)
\end{array}\right]}_{\mathbf{C}_{2 k}^{r}(n)} \mathbf{d}_{2 k}^{A}(n)+\mathbf{z}_{2 k}^{r A}(n)
$$

where

$$
\mathbf{y}_{2 k}^{r A}(n)=\left[\begin{array}{c}
y_{2 k}^{r}(n) \\
-y_{2 k+1}^{r *}(n)
\end{array}\right], \mathbf{d}_{2 k}^{A}(n)=\left[\begin{array}{c}
d_{2 k}(n) \\
-d_{2 k+1}^{*}(n)
\end{array}\right]
$$

and

$$
\mathbf{z}_{2 k}^{r A}=\left[\begin{array}{c}
z_{2 k}^{r}(n) \\
-z_{2 k+1}^{r *}(n)
\end{array}\right]
$$

represents zero-mean additive noise components. If $M_{R}>1$ receiving elements are used, their 
signals can be arranged into a single vector, so that the system is fully described by

$$
\underbrace{\left[\begin{array}{c}
\mathbf{y}_{2 k}^{1 A}(n) \\
\vdots \\
\mathbf{y}_{2 k}^{M_{R} A}(n)
\end{array}\right]}_{\mathbf{y}_{2 k}^{A}(n)}=\underbrace{\left[\begin{array}{c}
\mathbf{C}_{2 k}^{1}(n) \\
\vdots \\
\mathbf{C}_{2 k}^{M_{R}}(n)
\end{array}\right]}_{\mathbf{C}_{2 k}(n)} \mathbf{d}_{2 k}^{A}(n)+\underbrace{\left[\begin{array}{c}
\mathbf{z}_{2 k}^{1 A}(n) \\
\vdots \\
\mathbf{z}_{2 k}^{M_{R} A}(n)
\end{array}\right]}_{\mathbf{z}_{2 k}^{A}(n)}
$$

Based on this model, least squares (LS) data estimates are obtained as

$$
\hat{\mathbf{d}}_{2 k}^{A}(n)=\left[\mathbf{C}_{2 k}^{H}(n) \mathbf{C}_{2 k}(n)\right]^{-1} \mathbf{C}_{2 k}^{H}(n) \mathbf{y}_{2 k}^{A}(n)
$$

\subsubsection{Channel model}

We model the UWA channel as

$$
H_{k^{\prime}}^{t, r}(n)=\sum_{p} h_{p}^{t, r}(n) e^{-\mathrm{j} 2 \pi f_{k^{\prime}} \tau_{p}^{t, r}(n)}
$$

where $h_{p}^{t, r}(n)$ and $\tau_{p}^{t, r}(n)$ represent, respectively, the gain and delay of the $p$-th propagation path, and $f_{k^{\prime}}=f_{0}+k^{\prime} \Delta f$ is the the $k^{\prime}$-th carrier frequency. We further assume that the path gains are slowly varying with the block index $n$, and that the delays are subject to compression/dilatation caused by motion at a constant relative velocity $v^{t, r}$ that does not change over a certain number of OFDM blocks. The delay is consequently modeled as

$$
\tau_{p}^{t, r}(n)=\tau_{p}^{t, r}(n-1)-a^{t, r} T^{\prime}=\tau_{p}^{t, r}(0)-a^{t, r} n T^{\prime}
$$

where $a^{t, r}=v^{t, r} / c$ is the Doppler scaling factor. We are specifically interested in the case in which the receiving elements are co-located and the major cause of motion is the motion of the transmitter. One can then assume that $a^{t, r}=a^{t}[2]$.

Synchronization at the receiver is performed independently for each receiving element. The receiver's reference time $\tau_{0}^{r}(0)$ is inferred from the composite received signal and set to 0 . In general, one can have both $\tau_{0}^{1, r}(0) \neq 0$ and $\tau_{0}^{2, r}(0) \neq 0$, as the signals arriving from different transmitters may have traversed different distances. We note, however, that when the transmit elements are co-located and separated by only a few wavelengths $\lambda_{0}=c / f_{0}$, the difference in the arrival times $\Delta \tau_{0}^{r}=\left|\tau_{0}^{1, r}(0)-\tau_{0}^{2, r}(0)\right|$ will be on the order of $\lambda_{0} / c$, e.g. a fraction of a millisecond for $f_{0}$ on the order of a few $\mathrm{kHz}$. This delay difference is small enough that the resulting phase rotation of the transfer function $H_{k^{\prime}}^{t, r}(n)$ will be slow over the carriers. The effect of delay difference will be further quantified through numerical examples in Chapter 5.

Given the delays (4.5), let us decompose the transfer functions (4.4) as follows:

$$
H_{k^{\prime}}^{t, r}(n)=A_{k^{\prime}}^{t, r}(n) e^{\mathrm{j} \alpha_{k^{\prime}}^{t}(n)}
$$


where

$$
A_{k^{\prime}}^{t, r}(n)=\sum_{p} h_{p}^{t, r}(n) e^{-\mathrm{j} 2 \pi f_{k^{\prime}} \tau_{p}^{t, r}(0)}
$$

are the (complex-valued) gains, and

$$
\alpha_{k^{\prime}}^{t}(n)=2 \pi f_{k^{\prime}} a^{t} n T^{\prime}
$$

are the incremental phases of the two transmitters' channels. We note that the phases $2 \pi f_{k^{\prime}} \tau_{p}^{t, r}(0)$ are time-invariant, hence $A_{k^{\prime}}^{t, r}(n)$ are only slowly varying as dictated by the path gains $h_{p}^{t, r}(n)$, while the dominant cause of time variation in $H_{k^{\prime}}^{t, r}(n)$ are the phases $\alpha_{k^{\prime}}^{t}(n)$. We will use these facts in Sec.4.4 to design an adaptive channel tracking algorithm.

\subsubsection{The Alamouti assumption}

Extraction of the transmit diversity gain through summation of individual channel's energies, and simplicity of data detection without matrix inversion, form the essence of Alamouti processing. Our interest is to identify which situations the channel matrix satisfies the property

$$
\mathbf{C}_{2 k}^{r H}(n) \mathbf{C}_{2 k}^{r}(n)=\underbrace{\left(\left|H_{2 k}^{1, r}(n)\right|^{2}+\left|H_{2 k}^{2, r}(n)\right|^{2}\right)}_{E_{2 k}^{r}(n)} \mathbf{I}_{2}
$$

where $\mathbf{I}_{2}$ is the $2 \times 2$ identity matrix. We first derive the exact result of the matrix product

$\mathbf{C}_{2 k}^{r H}(n) \mathbf{C}_{2 k}^{r}(n)=\left[\begin{array}{cc}\left|H_{2 k}^{1, r}(n)\right|^{2}+\left|H_{2 k+1}^{2, r}(n)\right|^{2} & H_{2 k}^{1, r *}(n) H_{2 k}^{2, r}(n)-H_{2 k+1}^{1, r *}(n) H_{2 k+1}^{2, r}(n) \\ H_{2 k}^{1, r}(n) H_{2 k}^{2, r *}(n)-H_{2 k+1}^{1, r}(n) H_{2 k+1}^{2, r *}(n) & \left|H_{2 k+1}^{1, r}(n)\right|^{2}+\left|H_{2 k}^{2, r}(n)\right|^{2}\end{array}\right]$

from which, according to the desired structure (4.9), two equations arise

$$
\begin{aligned}
\left|H_{2 k}^{1, r}(n)\right|^{2}+\left|H_{2 k+1}^{2, r}(n)\right|^{2} & =\left|H_{2 k+1}^{1, r}(n)\right|^{2}+\left|H_{2 k}^{2, r}(n)\right|^{2} \\
H_{2 k}^{1, r *}(n) H_{2 k}^{2, r}(n) & =H_{2 k+1}^{1, r *}(n) H_{2 k+1}^{2, r}(n)
\end{aligned}
$$

The first equation coincides with the OFDM design principles (Sec.2.1), as well as with the Alamouti assumption expressed for space-frequency coding, which states that the channel does not change much over two consecutive carriers:

$$
H_{2 k}^{t, r}(n) \approx H_{2 k+1}^{t, r}(n)
$$

This first constraint is inherently verified by assuming a properly designed OFDM system, i.e. $T \gg T_{m p}$. Moreover, provided that initial synchronization is sufficiently accurate with respect to each transmitter, such that $\Delta f \tau_{0}^{t, r}(0) \ll 1, \forall t, r$, neither channel will exhibit significant phase rotation across the carriers. As mentioned earlier, this is a reasonable assumption for co-located transmitters. 
The second assumption requires

$$
e^{2 \pi f_{k^{\prime}+1} n a^{1} T^{\prime}-2 \pi f_{k^{\prime}} n a^{1} T^{\prime}}=e^{2 \pi f_{k^{\prime}+1} n a^{2} T^{\prime}-2 \pi f_{k^{\prime}} n a^{2} T^{\prime}}
$$

considering that the algorithm will track and compensate the phase in each block, we can omit the index $n$, i.e.

$$
e^{2 \pi \Delta f a^{1} T^{\prime}}=e^{2 \pi \Delta f a^{2} T^{\prime}}
$$

this assumption will hold as well provided that (i) synchronization is precise, i.e. $\tau_{0}^{t, r}(0) \approx 0$, (ii) $\Delta f T^{\prime} \sim 1$, and (iii) the residual Doppler factors $a^{t}$ typically do not exceed $10^{-4}$ within a single frame.

When both (4.11) and (4.12) verify, the channel matrix satisfies the property

$$
\mathbf{C}_{2 k}^{r H}(n) \mathbf{C}_{2 k}^{r}(n)=\underbrace{\left(\left|H_{2 k}^{1, r}(n)\right|^{2}+\left|H_{2 k}^{2, r}(n)\right|^{2}\right)}_{E_{2 k}^{r}(n)} \mathbf{I}_{2}+\mathbf{W}
$$

However, since (4.16) is based on assumptions that are clearly dependent on the channel geometry, this result may not be well justified in all the scenarios. To characterize this inaccuracy, we have included a matrix $\mathbf{W}$, which characterizes the additional mean squared error (MSE) introduced in the decoding process due to the inaccuracy of the assumptions.

We will show with a channel simulation that the effects of $\mathbf{W}$ are not significant. Let us consider an scenario with two transmitters and one receiver, where the transmitter sends Alamouti symbols coded along frequency carriers. The receiver assumes (4.13) and (4.15), and carries out the LS data estimation as follows

$$
\hat{\mathbf{d}}_{2 k}^{A}(n)=\frac{1}{\sum_{r} E_{2 k}^{r}(n)} \mathbf{C}_{2 k}^{H}(n) \mathbf{y}_{2 k}^{A}(n)
$$

the signal traverses a computer-simulated channel which has the following geometry: water depth $20 \mathrm{~m}$, transmitter and receiver at half depth, and link distance of $1 \mathrm{~km}$. The channel impulse response presents a multipath spread around $6 \mathrm{~ms}$. We evaluate the MSE introduced by the matrix $\mathbf{W}$ as a function of the following parameters:

- Synchronization mismatch: $\left|\tau_{0}^{2, r}(0)-\tau_{0}^{1, r}(0)\right|$.

- OFDM design: the product $\Delta f T_{m p}$.

Figure 4.1 illustrates the evolution of the MSE as both the product $\Delta f T_{m p}$ and the desynchronization between transmitters grow. It is clear that for a fixed $T_{m p}$ the performance decreases as both the delay $\left|\tau_{0}^{2, r}(0)-\tau_{0}^{1, r}(0)\right|$ and the subcarrier spacing $\Delta f$ increase. Data detection is well accomplished with delays up to $1 \mathrm{~ms}$ and frequency spacings on the order of $25 \mathrm{~Hz}$. In these cases, the use of simplified detection represents only a loss of -15 dB MSE or less. Provided that these delays and frequency spacings are typically found in actual experiments, and taking into account that in the more favorable conditions the actual obtained MSE is usually above $-15 \mathrm{~dB}$, we will no longer consider the effects of $\mathbf{W}$. 


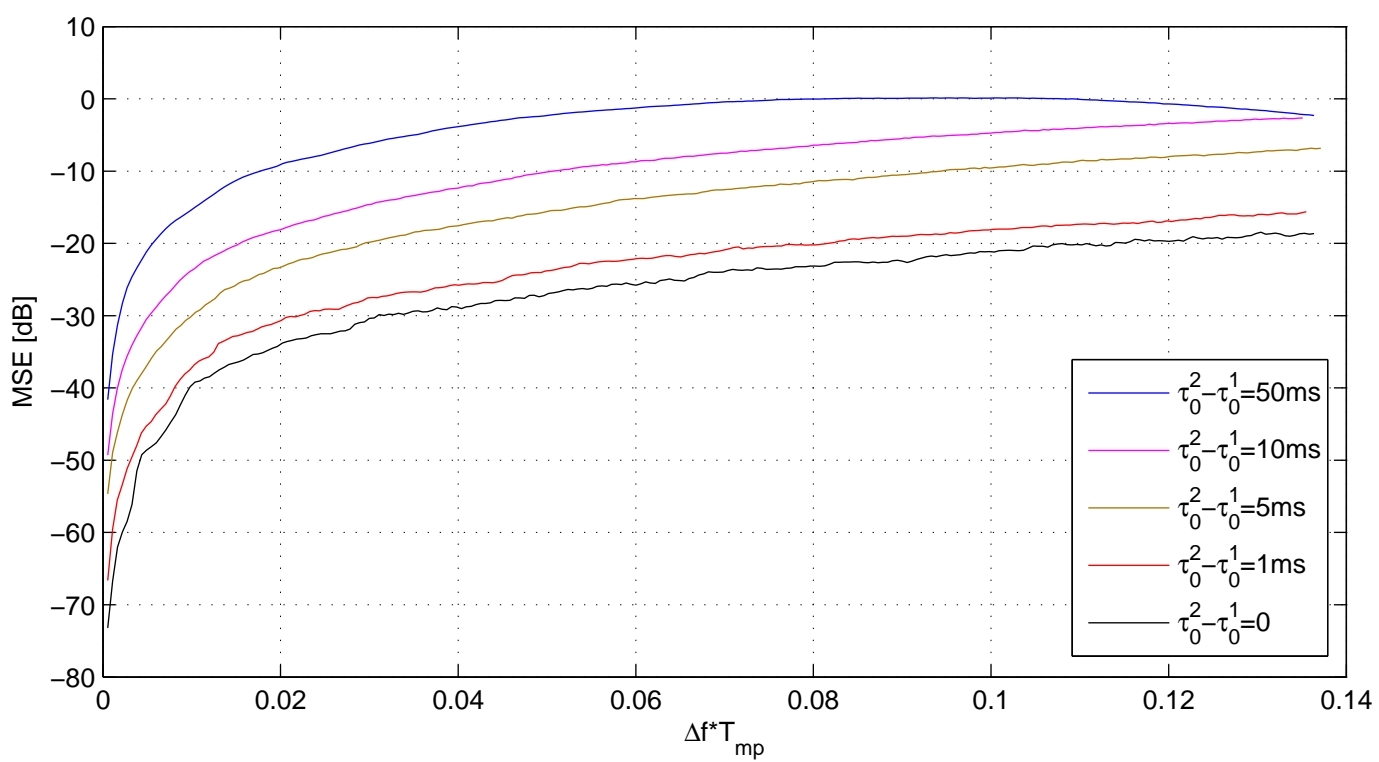

Figure 4.1: MSE introduced by the inaccuracy of the Alamouti assumptions.

\subsection{Transmitter description}

Our transmitter is based on an OFDM modulation in which the symbols are Alamouti-coded along frequency carriers. Besides the simplicity of the modulation, the distortion produced by the medium requires the OFDM blocks to be arranged in a frame structure [7], in order to allow for easy Doppler compensation at the receiver stage. The signals generated by our transmitter were recorded as audio files and sent to the Woods Hole Oceanographic Institution (WHOI), where our system was tested in an actual underwater scenario. In simulation, however, these files were directly processed in the laboratory, with the same channel distortions observed in the experiment applied to them.

\subsubsection{OFDM block}

The OFDM block is generated from a bit source, as described in Chap. 2. The bits coming from the source are first grouped in codewords and converted into QPSK symbols. Thereafter, the symbols that belong to the same codeword are interleaved in frequency. Finally, they are coded following the Alamouti scheme and a different OFDM signal is generated for each transmitter. Specifically, the steps illustrated in Fig. 4.2 are:

1. Channel coding: The bits are grouped in multiples of nine. Each 9-bit group is converted into a fourteen bit codeword using a Hamming $(14,9)$ dictionary, i.e. seven QPSK symbols are obtained from each 9-bit codeword.

2. Frequency interleaving: Consists of spacing as much as possible along the symbols that belong to the same codeword. To do so, the transmitter places each symbol of the same 
codeword every $K / 7$ carriers. If $K$ is not a multiple of 7 , the remaining carriers are padded until full length.

3. Alamouti code: Once the symbols are interleaved, the Alamouti code is applied. Two vectors of $K$ symbols result from this step, one for each transmitter.

4. IFFT: Finally, a $8 K$-size IFFT operation is performed to obtain the OFDM blocks that will be transmitted.

The employed transmitter configurations are summarized in Table 4.1.

Table 4.1: OFDM modulation parameters

\begin{tabular}{|l|l|}
\hline Bandwidth, $B$ & $4883 \mathrm{~Hz}$ \\
\hline First carrier frequency, $f_{0}$ & $10580 \mathrm{~Hz}$ \\
\hline Sampling frequency, $f_{s}$ & $39062 \mathrm{~Hz}$ \\
\hline Number of carriers, $K$ & $64,128,256,512,1024$ \\
\hline Carrier spacing, $\Delta f[\mathrm{~Hz}]$ & $76,38,19,10,5$ \\
\hline OFDM Block duration, $T[\mathrm{~ms}]$ & $13,26,52,104,210$ \\
\hline Channel code & Hamming $(14,9)$ \\
\hline
\end{tabular}

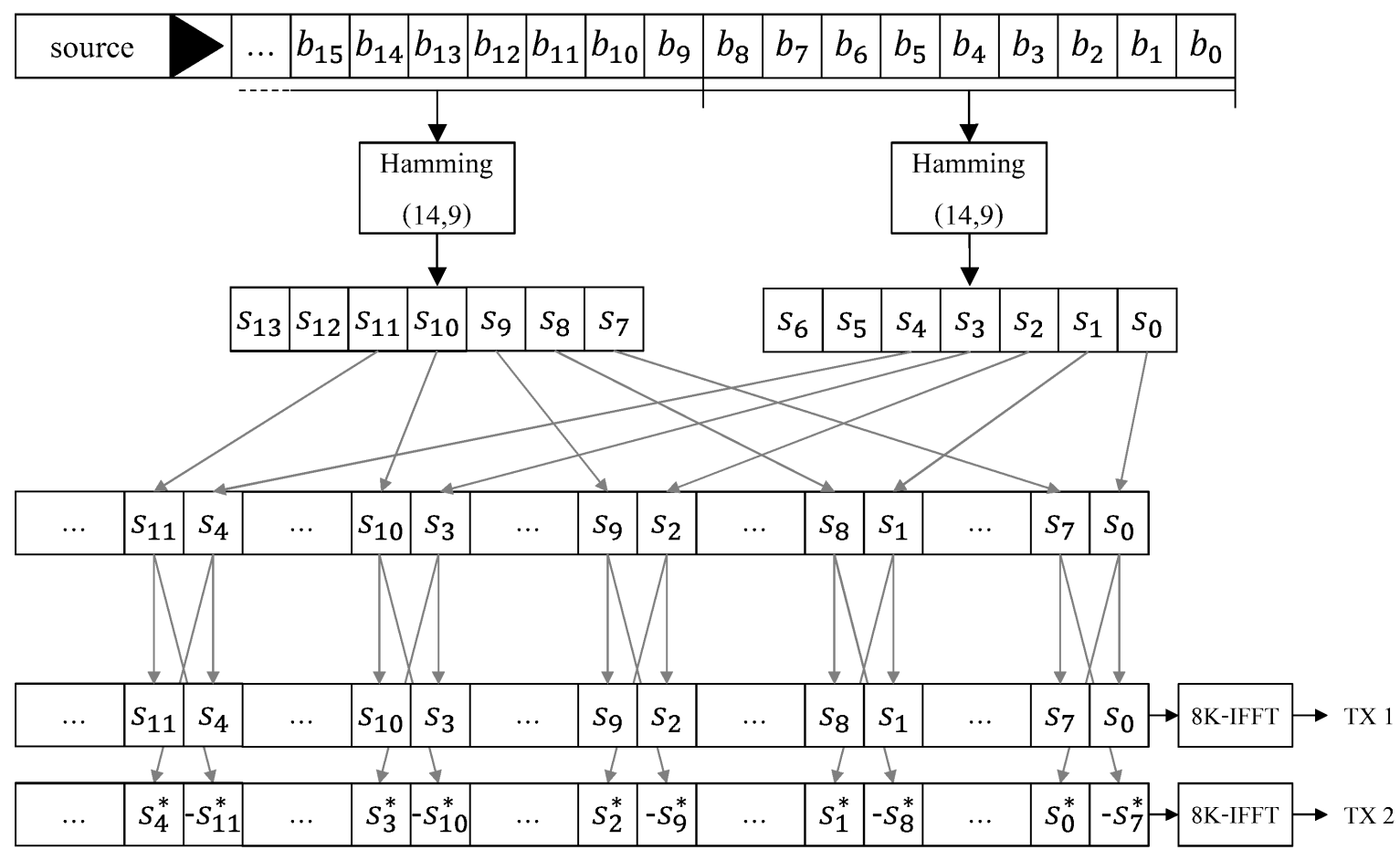

Figure 4.2: Alamouti-OFDM transmitter scheme. 


\subsubsection{Frame structure}

During the transmissions, the OFDM blocks are arranged into frames. These frames are basically a succession of OFDM symbols, each spaced by a guard interval of duration $T_{g}$. Eventually, a high resolution probe is inserted in order to aid the receiver synchronization and Doppler compensation processes. The period between synchronization blocks has to be determined as a function of the expected Doppler distortion. Concretely, one should only allow a maximum Doppler variation between sync blocks on the order of $10^{-4}$ if the convergence of the adaptive tracking algorithm is to be maintained. Figure 4.3 shows the employed frame structure.

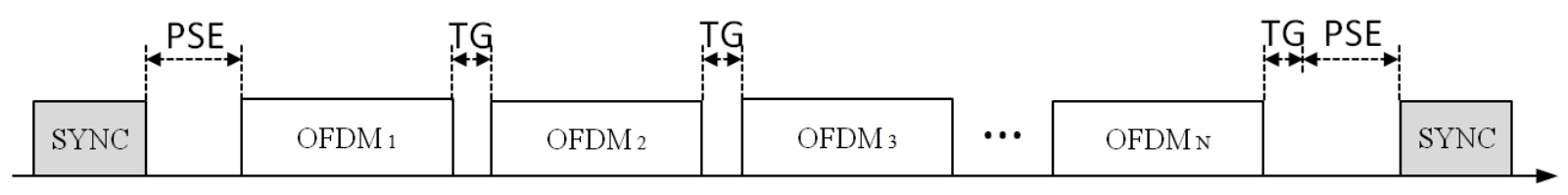

Figure 4.3: OFDM frame scheme.

Our interest is to evaluate the system behaviour as the number of carriers varies. To do so, we transmit several frames, each containing the same number of QPSK symbols $N_{d}$, whose OFDM symbols have different number of carriers ${ }^{1}$, i.e. different $K$. The $K$ values are sorted in ascending order, i.e. the first $N_{d}$ symbols are transmitted in blocks of $K=64$, followed by $N_{d}$ symbols in blocks of $K=128$, and so on. The synchronization blocks are inserted at the beginning and the end of each OFDM configuration. The specific parameters used in our experiments are detailed in Table 4.2 .

Table 4.2: OFDM frame parameters

\begin{tabular}{|l|l|}
\hline Guard interval, $T_{g}$ & $16 \mathrm{~ms}$ \\
\hline Sync guard interval, $T_{\mathrm{PSE}}$ & $32 \mathrm{~ms}$ \\
\hline Symbols per frame, $N_{d}$ & $8192 \mathrm{QPSK}$ \\
\hline Number of carriers, $K$ & $64,128,256,512,1024$ \\
\hline Blocks per frame, $N$ & $128,64,32,16,8$ \\
\hline Bitrate, $R$ [kbps] & $4.3,5.9,7.2,8.1,8.7$ \\
\hline
\end{tabular}

Figure 4.4 shows an actual transmitted frame. Note that all configurations bear the same number of symbols. However, higher $K$ values require less time to transmit them all because of the reduced amount of guard intervals.

\subsection{Front-end processing at the receiver}

This section contains a detailed description of the front-end processing stage at the receiver. When the transmitted signal reaches the receiver, it has been altered by the channel and the relative

\footnotetext{
${ }^{1} \mathrm{~A}$ real-time implementation would not be designed in this manner.
} 


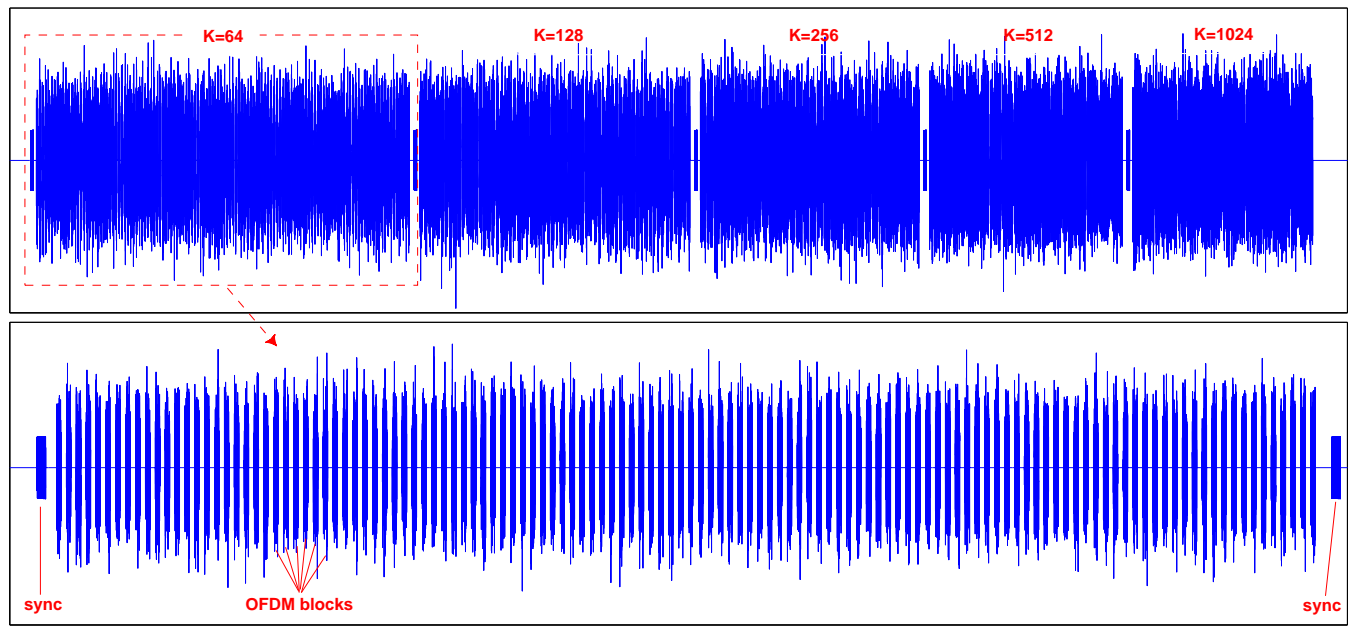

Figure 4.4: OFDM frame example.

motion between transmitter and receiver. The objective of the pre-processing stage is to remove the Doppler distortion, synchronize the signal and separate the OFDM symbols. The symbols are thereafter fed into the decoder algorithm.

\subsubsection{Signal detection}

The beginning of a frame is detected at the receiver by means of the correlation between the signal received from the medium and the synchronization block. When a synchronization block is received, the correlation output presents a function whose shape is similar to a delta. An amplitude threshold is set to trigger the start of the receiver algorithm.

The receiver, which has knowledge of the frame structure, takes the detected synchronization blocks and saves them to the memory. The first step is to revert the exponential product applied by the transmitter so as to downconvert the signal to baseband. After that, the signal is filtered to remove both the noise present in the unused bands and the spectral copies that appear as a consequence of the demodulation process. The filter is built with the following design specifications:

- Pass frequency: $F_{\text {pass }}=2.5 \mathrm{kHz}$

- Filter gain: $G=0 \mathrm{~dB}$

- Pass band maximum ripple: $A_{\text {pass }}=1 \mathrm{~dB}$

- Stop frequency: $F_{\text {stop }}=3 \mathrm{kHz}$

- Stop zone gain: $A_{\text {stop }}=-40 \mathrm{~dB}$

The filter response is represented in Figure 4.5.

Once the signal has been demodulated to baseband, we proceed to compensate the Doppler distortion that it may contain. 


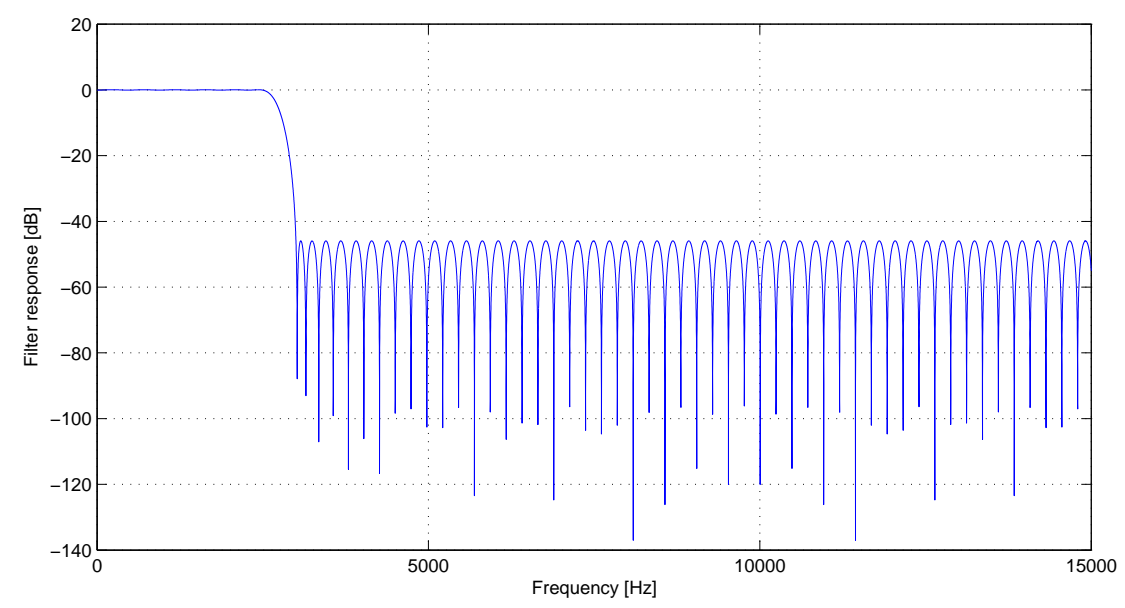

Figure 4.5: Downconverter filter response.

\subsubsection{Doppler compensation}

The most critical function of the pre-processing stage is the one that concerns Doppler compensation. In this section, we explain in detail the resampling algorithm, as well as how the Doppler factors are estimated.

Let us first model the Doppler effect produced by motion. Bearing in mind the multicarrier channel and Doppler shift models introduced in Sections 1.2 and 1.3, respectively, we express the received baseband OFDM signal as

$$
r(t)=\sum_{k=0}^{K-1} d_{k} e^{\mathrm{j} 2 \pi k \Delta f t}\left[\sum_{p} h_{p} e^{-\mathrm{j} 2 \pi f_{k^{\prime}} \tau_{p}} e^{\mathrm{j} 2 \pi a_{p} f_{k^{\prime}} t} \prod\left(\frac{t-\tau_{p}+a_{p} t}{T}\right)\right]+z(t)
$$

However, we will assume that every path has a similar Doppler factor $a_{p} \approx a$, so (4.18) simplifies to

$$
r(t)=\sum_{k=0}^{K-1} d_{k} e^{\mathrm{j} 2 \pi k \Delta f t} e^{\mathrm{j} 2 \pi a f_{k^{\prime}} t}\left[\sum_{p} h_{p} e^{-\mathrm{j} 2 \pi f_{k^{\prime}} \tau_{p}} \prod\left(\frac{t-\tau_{p}+a t}{T}\right)\right]+z(t)
$$

We note, based on the expression in (4.19), that two effects are present:

1. The signal received from each path is scaled in duration, from $T$ to $\frac{T}{1+a}$.

2. Each subcarrier experiences a frequency shift equal to $e^{\mathrm{j} 2 \pi a f_{k^{\prime}} t}$, which depends on the subcarrier frequency. These frequency-dependent shifts introduce strong intercarrier interference if an effective Doppler compensation scheme is not performed before the OFDM demodulation.

Based on this model, we apply a non-uniform Doppler compensation. This compensation can be performed either in passband or baseband. Given that the signals were already downconverted in the previous step, we choose to compensate the motion effects in baseband. 
The first step consists of Doppler factor estimation. This estimation relies on the preamble and the postamble of the frame, whose delay $T_{r x}$ is measured and compared with the nominal delay of the transmitted frame $T_{t x}$, i.e. the nominal frame duration. This way, the receiver infers how the received signal has been compressed or dilated by the channel:

$$
\hat{a}=\frac{T_{t x}}{\hat{T}_{r x}}-1
$$

Then, using the resampling factor obtained in (4.20) we resample the received waveform

$$
r^{\prime}(t)=r\left(\frac{t}{1+\hat{a}}\right)
$$

Thus, (4.19) becomes

$$
r^{\prime}(t)=\sum_{k=0}^{K-1} d_{k} e^{\mathrm{j} 2 \pi k \Delta f \frac{1+a}{1+\hat{a}} t} e^{\mathrm{j} 2 \pi \frac{a}{1+\hat{a}} f_{c} t}\left[\sum_{p} h_{p} e^{-\mathrm{j} 2 \pi f_{k^{\prime}} \tau_{p}} \prod\left(\frac{\frac{1+a}{1+\hat{a}} t-\tau_{p}}{T}\right)\right]+z(t)
$$

Since $\hat{a} \approx a$, we consider that $(1+a) /(1+\hat{a}) \approx 1$. Therefore, (4.22) reduces to

$$
r^{\prime}(t)=\sum_{k=0}^{K-1} d_{k} e^{\mathrm{j} 2 \pi k \Delta f t} e^{\mathrm{j} 2 \pi \frac{a}{1+\hat{a}} f_{c} t}\left[\sum_{p} h_{p} e^{-\mathrm{j} 2 \pi f_{k^{\prime}} \tau_{p}} \prod\left(\frac{t-\tau_{p}}{T}\right)\right]+z(t)
$$

So, the frequency dependent shift has been effectively compensated, leaving only a uniform residual shift $e^{\mathrm{j} 2 \pi \frac{a}{1+\hat{a}} f_{c} t}$ remaining.

\section{Doppler factor estimation}

The Doppler factor is estimated at the receiver using the method described by (4.20). This process involves considerable computational complexity since it requires continuous measurement of the cross correlation between the received signal and the known preamble. Moreover, the receiver must wait until the whole frame has been received to measure the total duration of the frame. The measured duration will then be compared with the nominal duration to infer the Doppler factor estimate $\hat{a}$.

At the beginning, the receiver only listens for activity in the channel. When a frame is detected, the preamble and postamble blocks are immediately stored in a buffer. At the same time, the signal containing the OFDM blocks is stored in an independent buffer for future processing. To locate the postamble, the receiver assumes that there is no Doppler distortion and captures 400 extra samples in both sides of the block, so as to accommodate the expected shifting. Figures 4.6 and 4.7 show the content of the preamble and postamble buffers, respectively.

To identify with precision the beginning of the block, the stored preamble and postamble are correlated with the transmitted probe. The received blocks, however, have been altered by the channel and, instead of showing a clear delta, its correlation results in the channel impulse 


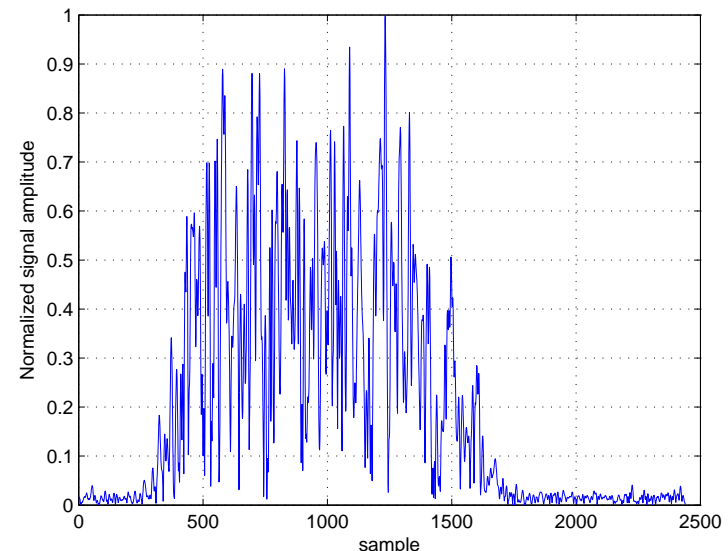

Figure 4.6: Received frame-preamble.

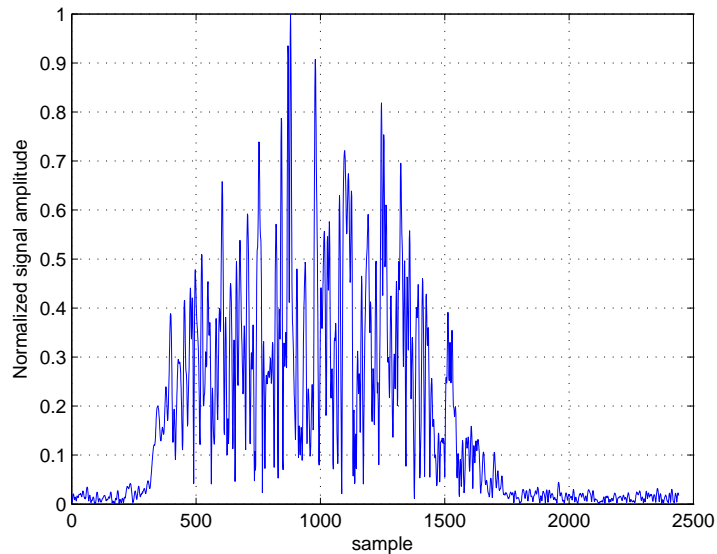

Figure 4.7: Received frame-postamble.

response (see figures 4.8 and 4.9). To recover the delta shape, we assume that the impulse response
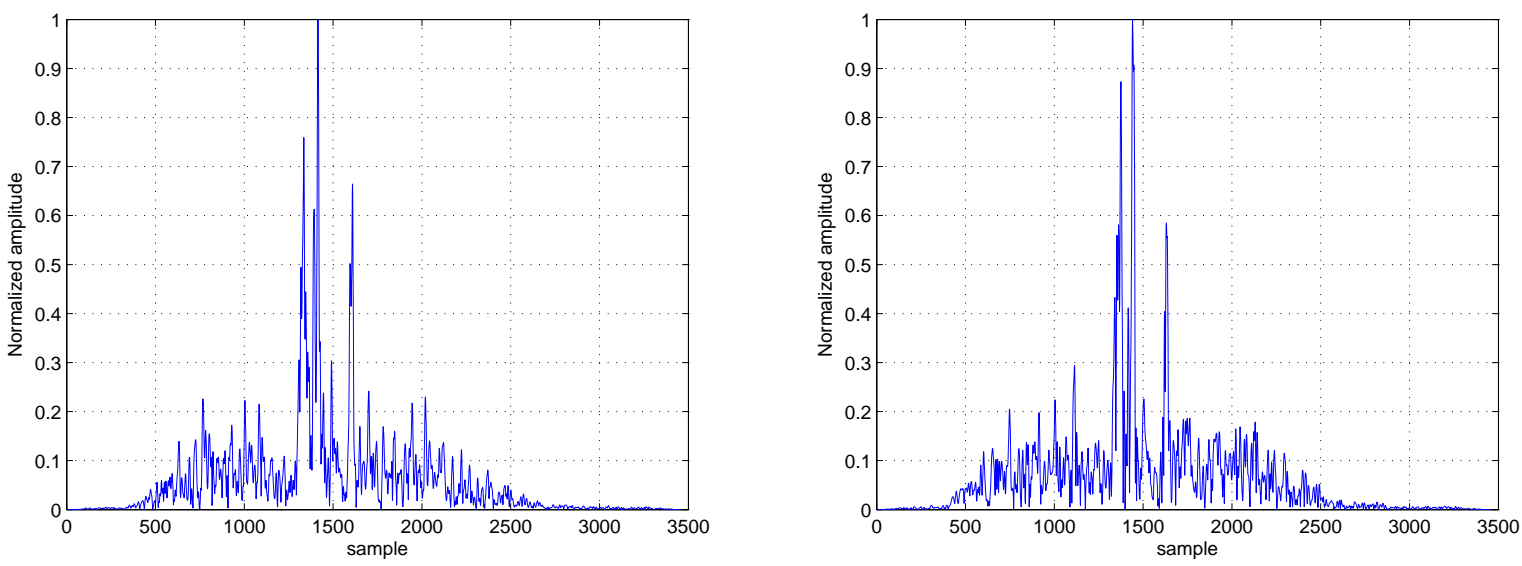

Figure 4.8: Cross correlation between sent and Figure 4.9: Cross correlation between sent and received preamble. received postamble.

has not changed significantly within the frame. Under this assumption, one can perform the cross correlation with the result of the previously correlated blocks. Doing so will compensate for the channel effect. Moreover, by setting the relative delay to zero in the exact point where $T_{r x}=T_{t x}$, one will directly obtain $\Delta \hat{T}=\hat{T}_{r x}-T_{t x}$ (the Doppler shift) at the point where the correlation presents its maximum. Figure 4.10 shows the result of the cross correlation, which effectively has a delta-like shape. Its maximum is located at the 27th sample. Consequently, the measured Doppler shift is $\Delta \hat{T}=27$ samples.

Before proceeding with the Doppler resampling algorithm, we refine the Doppler shift by taking the median of the set of estimated shifts from all the receivers. The reason for doing so is that eventually one receiver may have a bad measurement, probably as a consequence of increased channel variation. The median is an appropriate operation provided that we expect to measure 


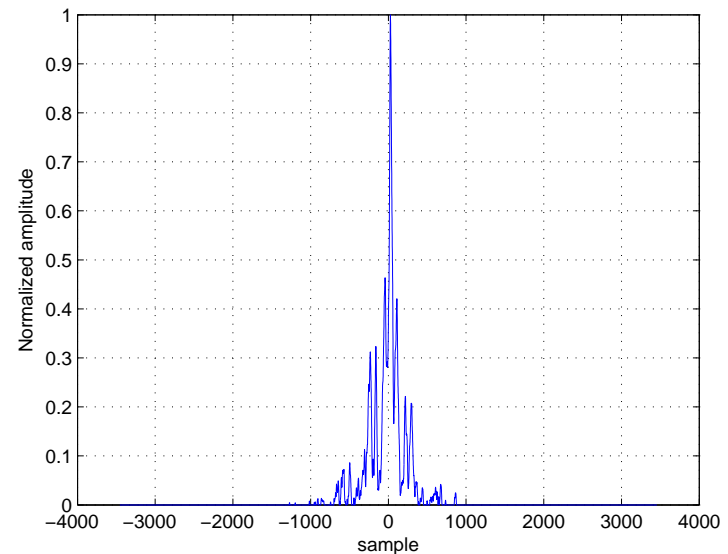

Figure 4.10: Cross correlation between previ- Figure 4.11: Detailed zoom. The cross correlaously correlated preamble and postamble.

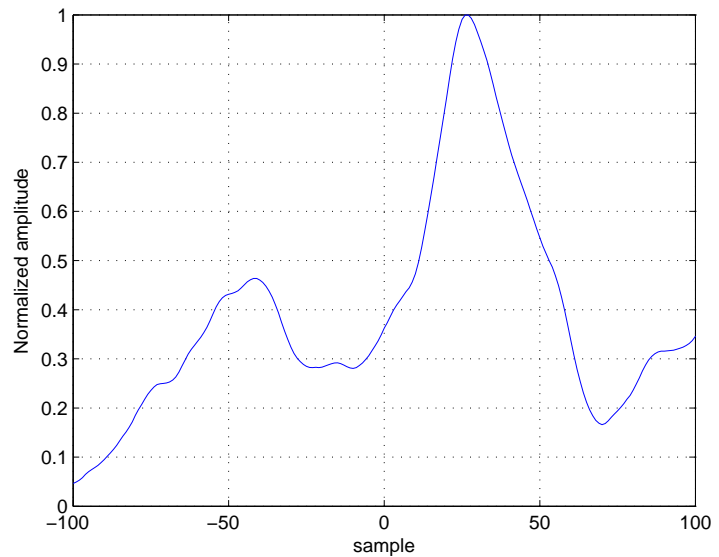

tion presents a maximum at sample 27.

the same Doppler shift in all the receivers. In this example, the measured Doppler shifts are shown in Table 4.3 .

Table 4.3: Measured Doppler shift (samples)

\begin{tabular}{|l|c|c|c|c|}
\hline & $K=64$ & $K=128$ & $K=256$ & $K=512$ \\
\hline Receiver 1 & 51 & 3 & -3 & 7 \\
\hline Receiver 2 & 51 & 2 & -2 & 7 \\
\hline Receiver 3 & 50 & 2 & -2 & 7 \\
\hline Receiver 4 & 50 & 2 & -2 & 7 \\
\hline Receiver 5 & 50 & 3 & -3 & 7 \\
\hline Receiver 6 & 51 & 3 & -4 & 7 \\
\hline Receiver 7 & 50 & 2 & -3 & 7 \\
\hline Receiver 8 & 49 & 2 & -3 & 7 \\
\hline Receiver 9 & 50 & 2 & -4 & 7 \\
\hline Receiver 10 & 50 & 2 & -4 & 7 \\
\hline Receiver 11 & 50 & 2 & -3 & 7 \\
\hline Receiver 12 & 50 & 2 & -3 & 7 \\
\hline \hline Measured $\Delta \hat{T}$ & 50 & 2 & -3 & 7 \\
\hline
\end{tabular}

The Doppler factor $\hat{a}$ for each $K$ is finally estimated as a function of $\Delta \hat{T}$

$$
\hat{a}=\frac{-\Delta \hat{T}}{T_{t x}+\Delta \hat{T}}
$$

\section{Resampling algorithm}

The resampling algorithm consists of a simple interpolation that effectively changes the signal time scale. The shifts produced at the beginning of the frame are usually much smaller than a sample. Consequently, the interpolation error is high if the resampled value is to be directly calculated 
with a linear interpolation. To address this issue, the data is previously interpolated with a factor 4, using a 9-sample lowpass interpolation filter. Thereafter, the time scale is corrected and the new signal values are obtained by linear interpolation (see Fig.4.12). The resampling process is carried out with the following Matlab code:

$\%$ original signal: y

yint $=\left[\right.$ interp $\left(\right.$ double $\left.\left.\left(y \cdot \cdot^{\prime}\right), 4\right) ; 0\right]$;

$\mathrm{x}=1: 4 /(1+$ af actor $): 4 *$ length $(\mathrm{y})$;

$\mathrm{x}_{-} \mathrm{floor}=\mathrm{floor}(\mathrm{x})$;

$\mathrm{x}_{-} \bmod =\mathrm{x}-\mathrm{x}_{-} \mathrm{floor}$;

yres $=\left(1-x_{-}\right.$mod $) . * y i n t\left(x_{-} f l o o r\right) . '+x_{-} \bmod . * y i n t\left(x_{-} f l o o r+1\right) \cdot{ }^{\prime} ;$

$\%$ resampled signal: yres

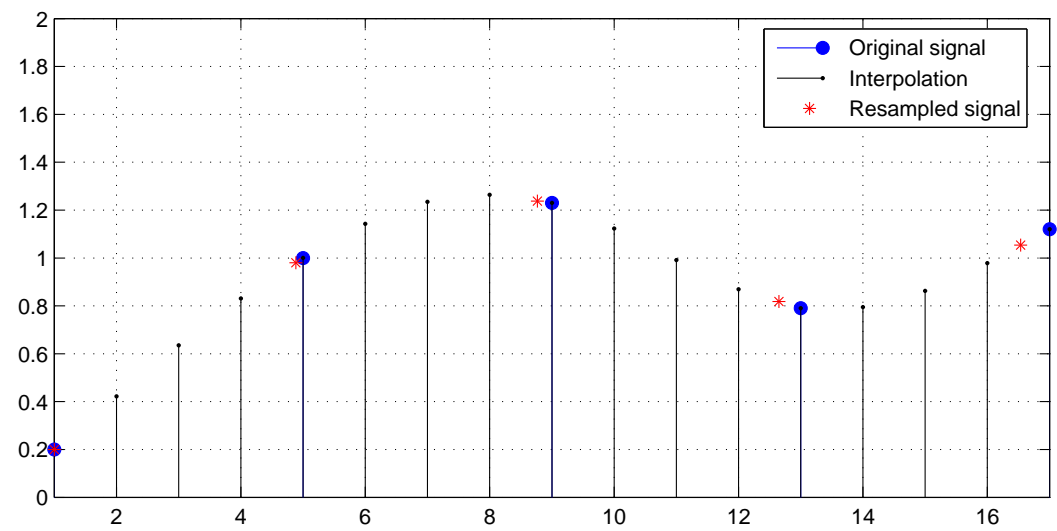

Figure 4.12: Doppler shift compensation using data interpolation.

\subsubsection{Time synchronization}

Syncronization is accomplished after the signal resampling process. Bearing in mind that the time distortion has been eliminated, the receiver can rely on the expected OFDM block durations and the frame timings. The output of the correlation (Fig. 4.10) provides a time reference of the beginning of the frame. Once the signal has been synchronized, the receiver extracts the OFDM blocks and stores them in separate buffers for future processing.

\subsection{Receiver algorithm}

The key to successful data detection is channel estimation. We focus on channel estimation method consisting of two steps: (i) an initial step, which is based on pilots only, and (ii) subsequent adaptation, which involves data detection as well. The initial step constitutes conventional, oneshot (non-adaptive) estimation, and can also be used alone, i.e. it can be applied repeatedly throughout a frame of OFDM blocks without engaging adaptation (time-smoothing). 
Channel estimation is performed independently for each receiving element, and it is based on the Alamouti assumption. If the Alamouti assumption holds, the received signal can be represented as

$$
\mathbf{y}_{2 k}^{r}(n)=\mathbf{D}_{2 k}(n) \underbrace{\left[\begin{array}{c}
A_{2 k}^{1, r}(n) e^{\mathrm{j} \alpha_{2 k}^{1}(n)} \\
A_{2 k}^{2, r}(n) e^{\mathrm{j} \alpha_{2 k}^{2}(n)}
\end{array}\right]}_{\mathbf{H}_{2 k}^{r}(n)}+\mathbf{z}_{2 k}^{r}(n)
$$

where

$$
\mathbf{D}_{2 k}(n)=\left[\begin{array}{cc}
d_{2 k}(n) & -d_{2 k+1}^{*}(n) \\
d_{2 k+1}(n) & d_{2 k}^{*}(n)
\end{array}\right]=\left[\begin{array}{c}
\mathbf{d}_{2 k}^{1 T}(n) \\
\mathbf{d}_{2 k}^{2 T}(n)
\end{array}\right]
$$

and

$$
\mathbf{y}_{2 k}^{r}(n)=\left[\begin{array}{c}
y_{2 k}^{r}(n) \\
y_{2 k+1}^{r}(n)
\end{array}\right], \mathbf{z}_{2 k}^{r}(n)=\left[\begin{array}{c}
z_{2 k}^{r}(n) \\
z_{2 k+1}^{r}(n)
\end{array}\right]
$$

Assuming unit-amplitude PSK symbols, we have that

$$
\frac{1}{2} \mathbf{D}_{2 k}^{H}(n) \mathbf{D}_{2 k}(n)=\mathbf{I}_{2}
$$

Hence, if a particular pair of data symbols is known, the LS channel estimate is obtained directly from (4.25) as

$$
\check{\mathbf{H}}_{2 k}^{r}(n)=\frac{1}{2} \mathbf{D}_{2 k}^{H}(n) \mathbf{y}_{2 k}^{r}(n)
$$

i.e.

$$
\check{H}_{2 k}^{t, r}(n)=\frac{1}{2} \mathbf{d}_{2 k}^{t H}(n) \mathbf{y}_{2 k}^{r}(n)
$$

\subsubsection{One-shot channel estimation}

Pilot-based channel estimation exploits the discrete Fourier relationship between the channel coefficients in the transfer function (TF) domain and the impulse response (IR) domain, where there are typically many fewer non-zero coefficients. To estimate a channel with $L$ non-zero IR coefficients, at least $L$ pilots are needed for each transmitter. Considering a system with a typical multipath spread of about $10 \mathrm{~ms}$ and a bandwidth of $10 \mathrm{kHz}$, the number of non-zero IR coefficients is on the order of 100 . For simplicity, $L$ is taken as a power of 2 , and pilot pairs are inserted evenly, i.e. every $K / L$ pairs of carriers.

TF coefficients of the pilot carriers are estimated using (4.27), and the inverse discrete Fourier transform (IDFT) is applied to obtain the IR coefficients ${ }^{2}$

$$
\check{h}_{m}^{t, r}(n)=\frac{1}{L} \sum_{l=0}^{L-1} \check{H}_{l K / L}^{t, r}(n) e^{\mathrm{j} 2 \pi \frac{l m}{L}}, \quad m=0 \ldots L-1
$$

\footnotetext{
${ }^{2}$ The IR coefficients are not to be confused with the path gains $h_{p}^{t, r}(n)$.
} 
or equivalently in the matrix form,

$$
\check{\mathbf{h}}^{t, r}(n)=\frac{1}{L} \mathbf{F}_{L}^{H} \check{\mathbf{H}}^{t, r}(n)
$$

where $\mathbf{F}_{L}$ is an appropriately defined DFT matrix.

\section{Sparse channel estimation - The LS-AT algorithm}

In an acoustic channel, it is often the case that the vector of IR coefficients $\check{\mathbf{h}}^{t, r}(n)$ is sparse, with only $J<L$ significant coefficients. Methods for sparse channel estimation, and in particular the OMP algorithm, have been shown to be very effective in such situations [9], [21], [22]. These methods typically provide a sparse solution $\overline{\mathbf{h}}^{t, r}(n)$ that best matches the model $\check{\mathbf{H}}^{t, r}(n)=\mathbf{F}_{L} \overline{\mathbf{h}}^{t, r}(n)$ for a given input $\check{\mathbf{H}}^{t, r}(n)$ and a desired degree of sparseness $J$.

As an alternative to the OMP method, we consider a method of least squares with adaptive thresholding. This method eliminates the need to set the desired degree of sparseness a-priori, while keeping the computational load at a minimum. The LS-AT algorithm uses the design value $T_{m p}$ as an upper bound of the multipath spread, and changes a truncation threshold $\gamma$ until the total delay spread $\check{T}_{m p}$ of the sparse solution $\overline{\mathbf{h}}^{t, r}(n)$ fits into the design value. The threshold is initially set to $\gamma=50 \%$ of the strongest coefficient's magnitude. The IR coefficients whose relative magnitude is below the threshold are discarded, and if the resulting delay spread is found to be less than the design value $T_{m p}$, the threshold is lowered. Otherwise, it is increased. The threshold values are assigned following a bisection method [23], in which the subgradient is computed as $\Delta \gamma=\operatorname{sign}\left(\hat{T}_{m p}-T_{m p}\right)$. See Figure 4.13 for an illustrative example.

The algorithm proceeds in this manner for a pre-determined minimum number of steps $S$. Thereafter, it continues if the threshold is to be raised further, and stops when a decreasing threshold is detected. The number of steps is chosen according to the desired resolution, $2^{-S}$. In the numerical analysis of Sec.5, we employ 20 steps and $T_{m p}$ equal to the guard interval. Similar approaches have been considered before [24],[25], where the truncation threshold is determined adaptively as a function of the noise power. Our algorithm is formalized in Alg.2.

Once the sparse impulse response $\overline{\mathbf{h}}^{t, r}(n)$ has been obtained, it is zero-padded to the full length $K$, and the TF coefficients on all the carriers are estimated as the DFT of the so-obtained $1 \times K$ vector $\hat{\mathbf{h}}^{t, r}(n){ }^{3}$

$$
\hat{\mathbf{H}}^{t, r}(n)=\mathbf{F}_{K} \hat{\mathbf{h}}^{t, r}(n)
$$

The TF coefficients are now used to form the channel matrices needed for data detection.

\footnotetext{
${ }^{3}$ Because the sparse IR has been obtained by removing samples from $\check{\mathbf{h}}^{t, r}(n)$, the resulting transfer function may contain distortion at the ends of the spectrum. To avoid this effect, null carriers can be added at the end of the LS estimates (4.28) and removed from $\hat{\mathbf{H}}^{t, r}$ after sparsing the impulse response.
} 

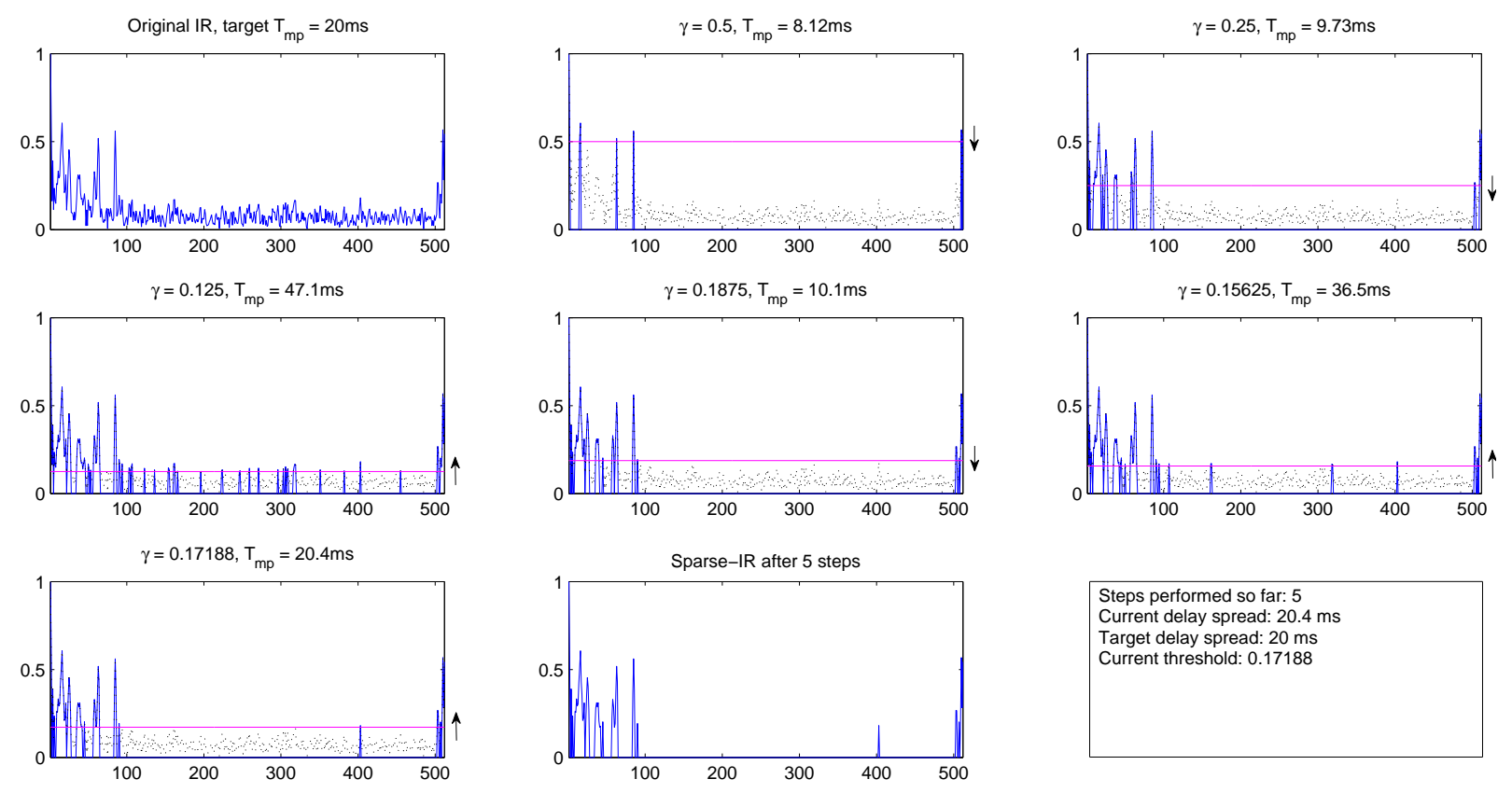

Figure 4.13: Adaptive thresholding example.

\section{A note on TF coefficients and the $\Delta f / 2$ correction}

The exact value of the initial observation for the first transmitter, ${ }^{4} \check{H}_{2 k}^{1, r}(n)$, which is used as the input to the channel estimator, is

$$
\begin{aligned}
\check{H}_{2 k}^{1, r}(n) & =\frac{1}{2}\left(H_{2 k}^{1, r}(n)+H_{2 k+1}^{1, r}(n)\right) \\
& +\frac{1}{2} d_{2 k+1}^{*}(n) d_{2 k}^{*}(n)\left(H_{2 k+1}^{2, r}(n)-H_{2 k}^{2, r}(n)\right) \\
& +\frac{1}{2}\left(d_{2 k}^{*}(n) z_{2 k}^{r}(n)+d_{2 k+1}^{*}(n) z_{2 k+1}^{r}(n)\right)
\end{aligned}
$$

Considering the fact that $H_{2 k+1}^{2, r}(n) \approx H_{2 k}^{2, r}(n)$, and that the input noise is zero-mean, we have that

$$
E\left\{\check{H}_{2 k}^{1, r}(n)\right\}=\frac{H_{2 k}^{1, r}+H_{2 k+1}^{1, r}}{2} \approx H_{f_{2 k}+\frac{\Delta f}{2}}^{1, r}(n)
$$

Hence, channel estimation will effectively yield a TF coefficient that lies mid-way between the carriers $2 k$ and $2 k+1$, and this fact can be exploited to refine the final estimate. To do so, one can compute the DFT (4.31) at twice the resolution, then select every other element of the so-obtained TF vector, starting with a delay of one.

\footnotetext{
${ }^{4} \mathrm{~A}$ similar relationship holds for the other transmitter.
} 


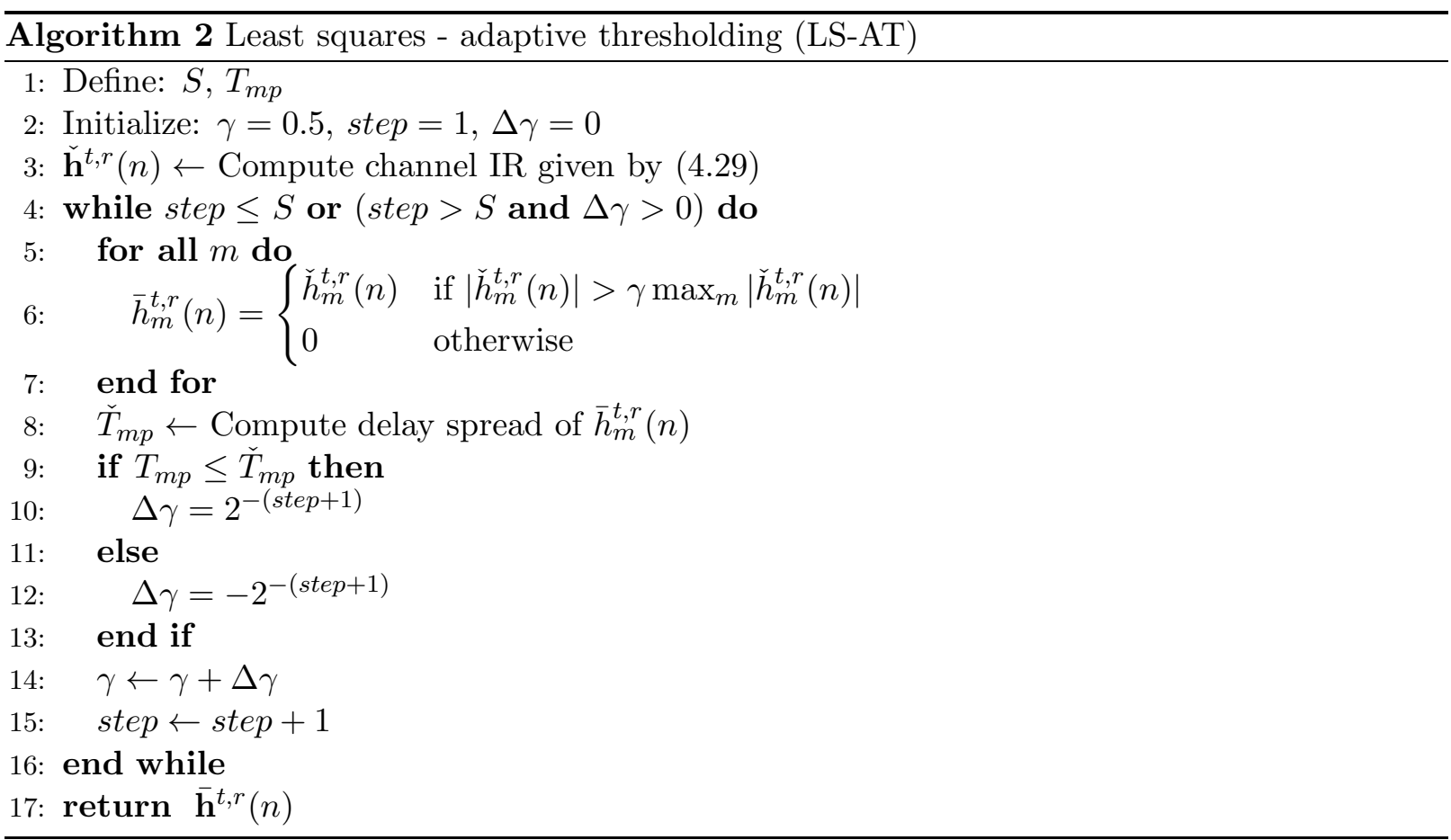

\section{Data detection}

Channel matrix $\hat{\mathbf{C}}_{2 k}(n)$ is now filled with the TF estimates $\hat{H}_{k^{\prime}}^{t, r}(n)$ according to the pattern (4.1), (4.2), and the data symbols are estimated according to (4.17) as

$$
\hat{\mathbf{d}}_{2 k}^{A}(n)=\frac{1}{\operatorname{tr}\left[\hat{\mathbf{C}}_{2 k}^{H}(n) \hat{\mathbf{C}}_{2 k}(n)\right]} \hat{\mathbf{C}}_{2 k}^{H}(n) \mathbf{y}_{2 k}^{A}(n)
$$

These estimates are fed to the decoder if additional channel coding is used, or used directly to make hard decisions. In either case, the process of decision making is denoted as

$$
\tilde{\mathbf{d}}_{2 k}^{A}(n)=\operatorname{Dec}\left[\hat{\mathbf{d}}_{2 k}^{A}(n)\right]
$$

\subsubsection{Adaptive channel estimation}

The goal of adaptive channel estimation is to exploit the time-correlation present in the channel so as to reduce the pilot overhead. To do so, we draw on the earlier channel decomposition into the slowly-varying gains $A_{k^{\prime}}^{t, r}(n)$, and phases $\alpha_{k^{\prime}}^{t}(n)$, whose variation in time is dictated by (possibly slowly-varying) Doppler factors $a^{t}(n)$. We target these sets of parameters individually in order to accomplish effective channel tracking. The adaptive algorithm proceeds in several steps, carried out for each block $n$. A block diagram of the adaptive receiver is shown in Figure 4.14. 


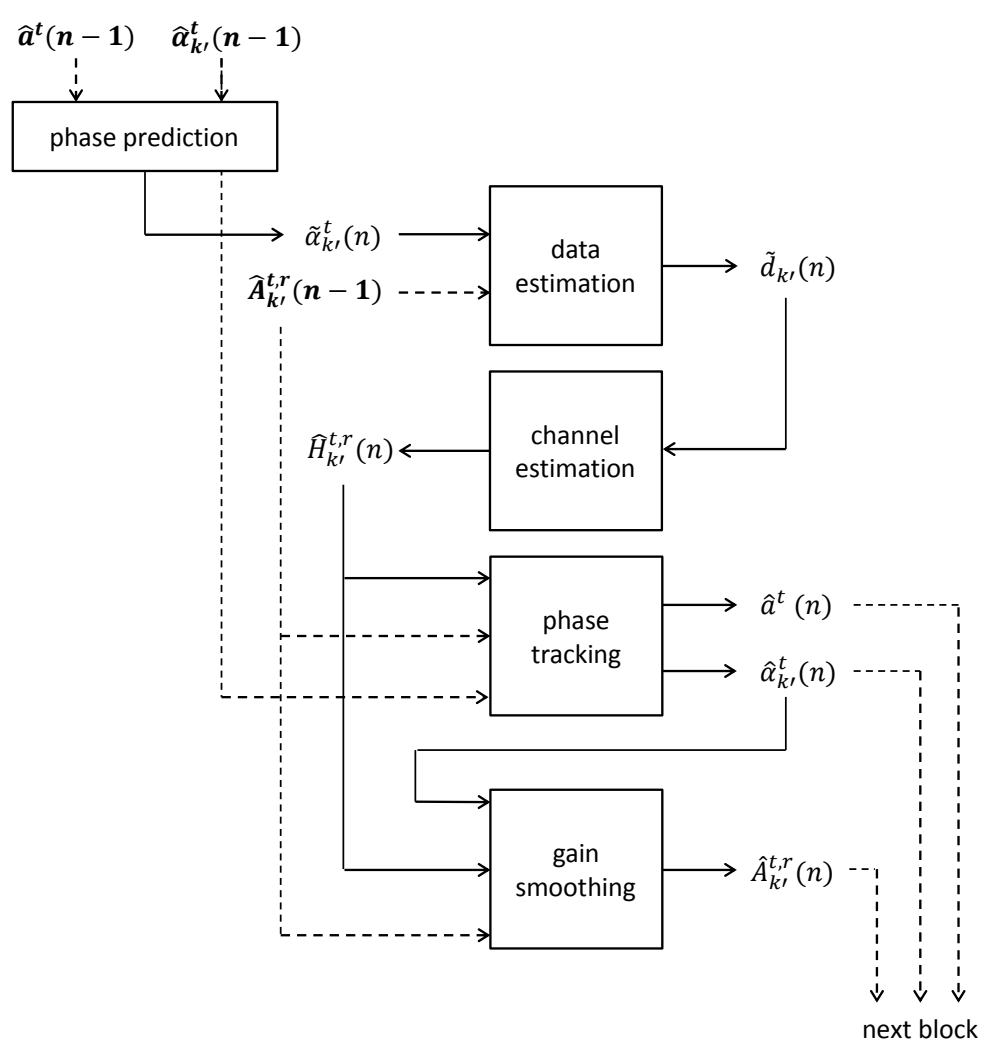

Figure 4.14: Block diagram of the adaptive receiver algorithm.

\section{Decision making}

Let us assume that predictions $\tilde{A}_{k^{\prime}}^{t, r}(n)$ and $\tilde{\alpha}_{k^{\prime}}^{t}(n)$, made at the end of a previous block from the estimates $\hat{A}_{k^{\prime}}^{t, r}(n-1)$ and $\hat{\alpha}_{k^{\prime}}^{t}(n-1)$, are available at the beginning of the current block $n$. These predictions are used to form the channel matrices $\tilde{\mathbf{C}}_{2 k}(n), k=0, \ldots K / 2-1$, which are in turn used to make symbol decisions

$$
\tilde{\mathbf{d}}_{2 k}^{A}(n)=\operatorname{Dec}\left[\frac{1}{\operatorname{tr}\left[\tilde{\mathbf{C}}_{2 k}^{H}(n) \tilde{\mathbf{C}}_{2 k}(n)\right]} \tilde{\mathbf{C}}_{2 k}^{H}(n) \mathbf{y}_{2 k}^{A}(n)\right]
$$

The symbol decisions are now treated as pilots, of which there may be as many as $L=K$, and they are used to update the phases and the channel estimates.

\section{Sparse channel estimation}

Let us denote the chosen channel estimation algorithm, be it OMP, LS-AT or similar, by $\mathcal{C} \mathcal{E}(\cdot)$. This algorithm is applied to obtain the one-shot channel estimate with resolution $K$ :

$$
\hat{\mathbf{H}}^{t, r}(n)=\mathcal{C E}\left(\left\{\tilde{\mathbf{d}}_{2 k}^{t H} \mathbf{y}_{2 k}^{r}(n)\right\}_{k=0}^{K / 2-1}\right)
$$




\section{Phase tracking}

To update the phases, we measure the phase difference (angle $\angle(\cdot)$ ) between the estimates made for the current block (4.37) and the outdated estimates from the previous block:

$$
\Delta \alpha_{k^{\prime}}^{t}(n)=\angle \sum_{r=1}^{M_{R}} \frac{\hat{H}_{k^{\prime}}^{t, r}(n)}{\tilde{A}_{k^{\prime}}^{t, r}(n) e^{j \hat{\alpha}_{k^{\prime}}^{t}(n-1)}}
$$

The phase difference thus is obtained and the Doppler factors for the current block are now estimated as

$$
\hat{a}^{t}(n)=\frac{1}{K} \sum_{k^{\prime}=0}^{K-1} \frac{\Delta \alpha_{k^{\prime}}^{t}(n)}{2 \pi f_{k^{\prime}} T^{\prime}}
$$

The phases are finally updated as

$$
\hat{\alpha}_{k^{\prime}}^{t}(n)=\hat{\alpha}_{k^{\prime}}^{t}(n-1)+2 \pi \hat{a}^{t}(n) f_{k^{\prime}} T^{\prime}
$$

If phase tracking/compensation throughout blocks is not performed, the received symbols suffer from a severe frequency-dependent phase rotation (Figure 4.15) that clearly reduces the receiver performance.
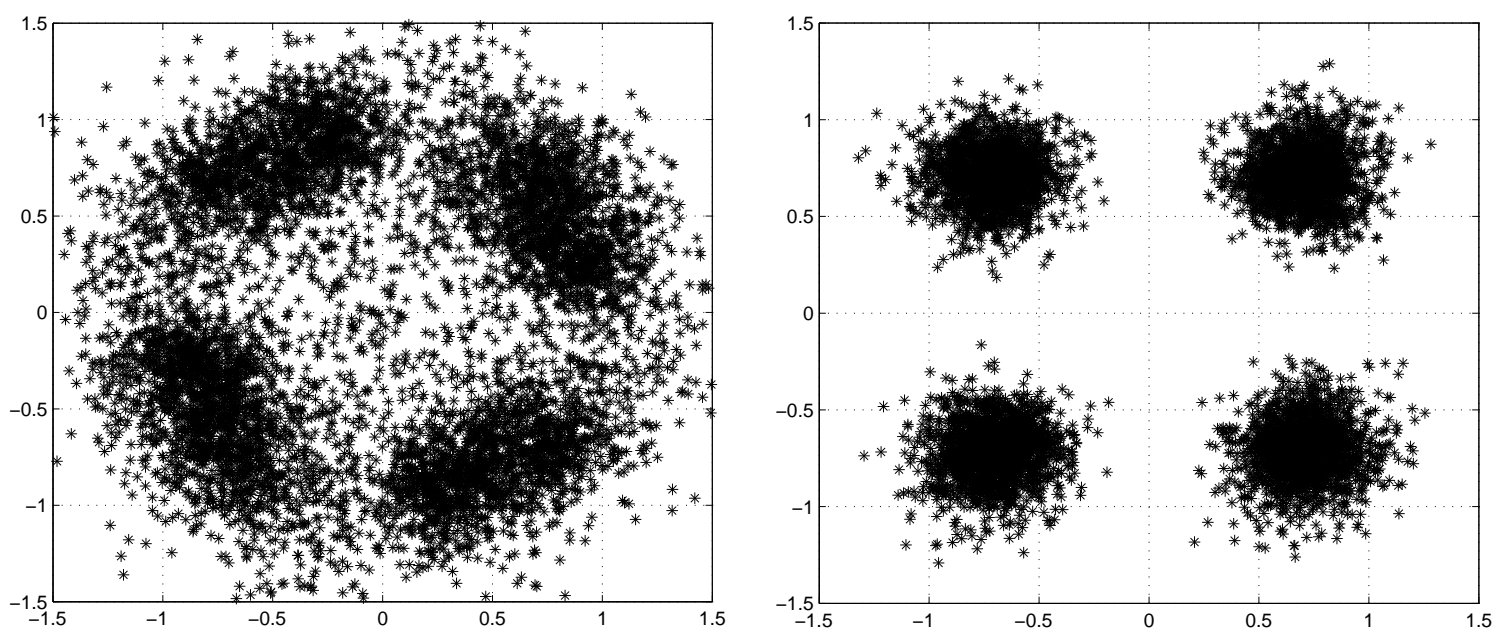

Figure 4.15: Scatter plot of real data using a receiver without (left) and with (right) phase tracking.

Note also that the phase prediction method is effective since the Doppler factor varies smoothly from one block to another. Figure 4.16 shows this smooth variation, as well as the fact that the Doppler factor variation is the same among receivers, but not necessarily among transmitters. 

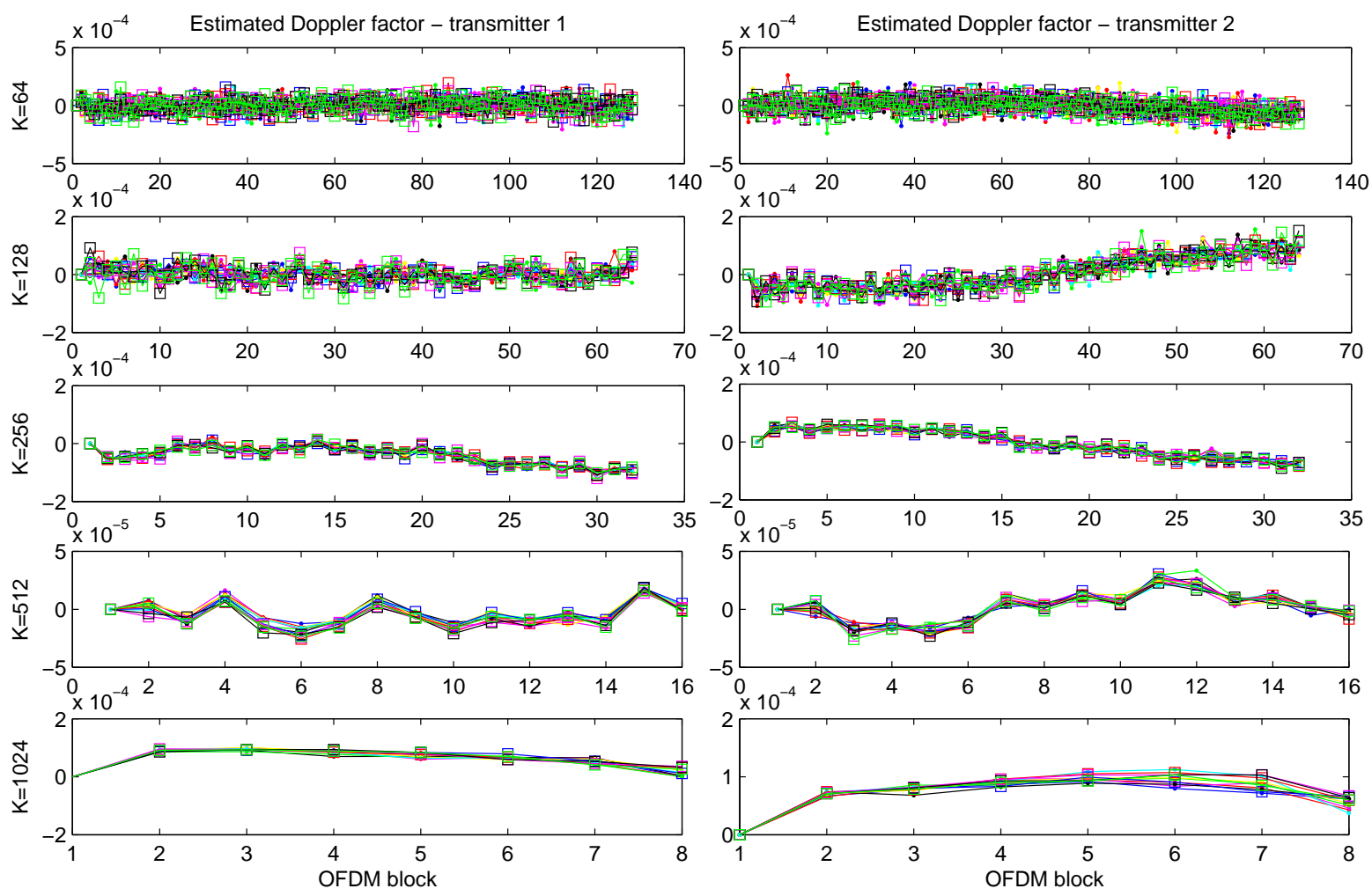

Figure 4.16: Measured Doppler evolution in experimental data for transmitters 1 and 2. Each line represents the residual Doppler observed in one receiving element.

\section{Channel tracking}

The updated $\hat{\alpha}_{k^{\prime}}^{t}(n)$ are now used to compensate for the time-varying phase of $\hat{H}_{k^{\prime}}^{t, r}(n)$ and the channel gains are updated as

$$
\hat{A}_{k^{\prime}}^{t, r}(n)=\lambda \hat{A}_{k^{\prime}}^{t, r}(n-1)+(1-\lambda) \hat{H}_{k^{\prime}}^{t, r}(n) e^{-\mathrm{j} \hat{\alpha}_{k^{\prime}}^{t}(n)}
$$

where $\lambda \in[0,1]$.

The channel gains $\hat{A}_{k^{\prime}}^{t, r}(n)$ are assumed to be very slowly varying from one block to another, provided that they only depend on the variation of the path gains. We take advantage of this slow variation by averaging the channel gain with an adaptation factor $\lambda$. A snapshot of the channel gain is shown in figure 4.17. The lines in the figure represent the channel gain for 16 consecutive OFDM blocks.

One may also notice that the adaptation factor must be set as a function of the expected channel variation. For example, values of $\lambda$ close to 1 rely more on the channel estimates made on previous blocks, whereas for $\lambda=0$ the receiver does not exploit the channel correlation. Four experimental transmissions with $K=256$ carriers, where the channel variation was especially severe, have been processed using different values of $\lambda$ ranging from 0 to 1 in steps of 0.1 . The 


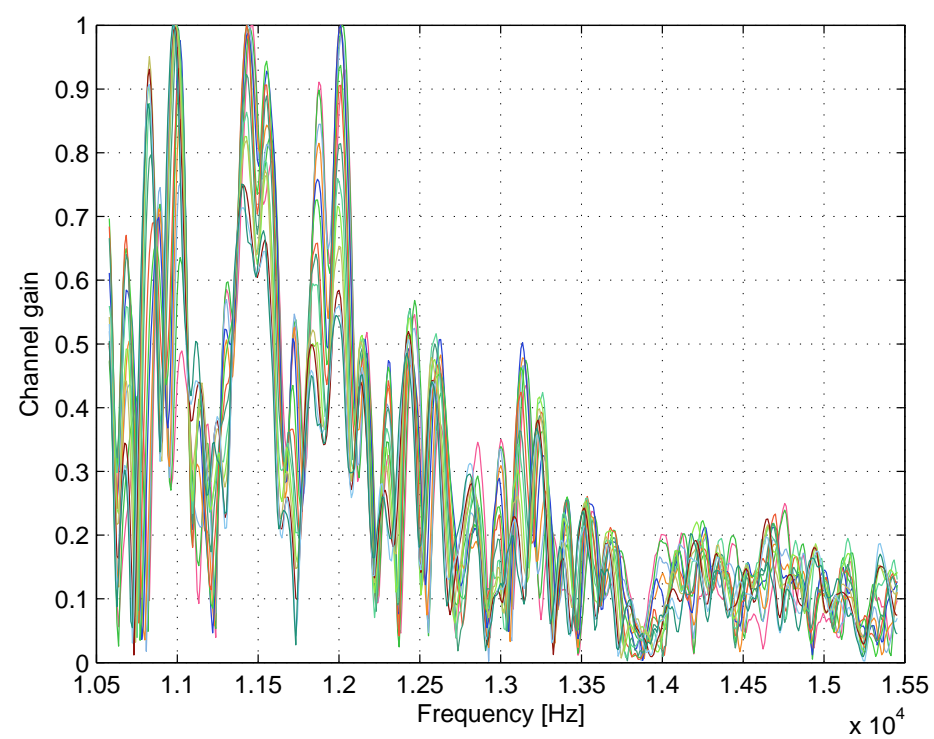

Figure 4.17: Channel gain variation during one OFDM frame, obtained from experimental data.

result is illustrated in Figures 4.18 and 4.19 with measures of the bit error rate and the mean squared error, respectively. Dashed lines represent how the adaptation factor affects each individual transmission, while the solid line represents the mean among them. Clearly, the best system performance is achieved with values between $\lambda=0.2$ and $\lambda=0.5$. The value employed in the experimental receiver is $\lambda=0.4$.

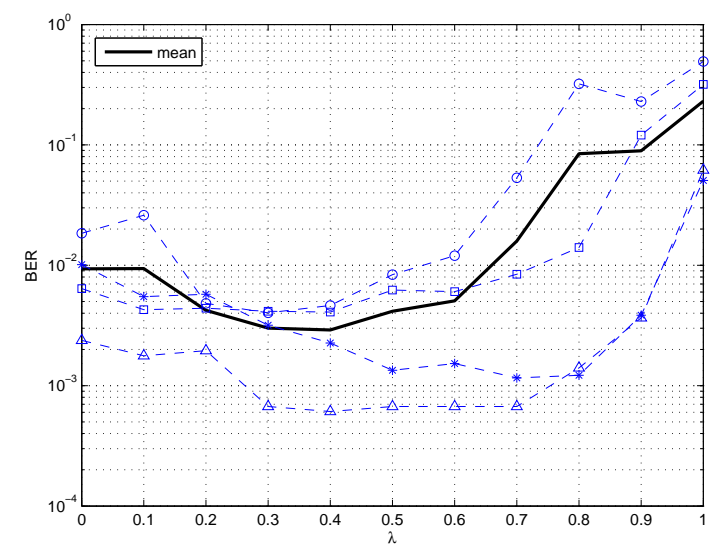

Figure 4.18: BER vs adaptation factor $\lambda$.

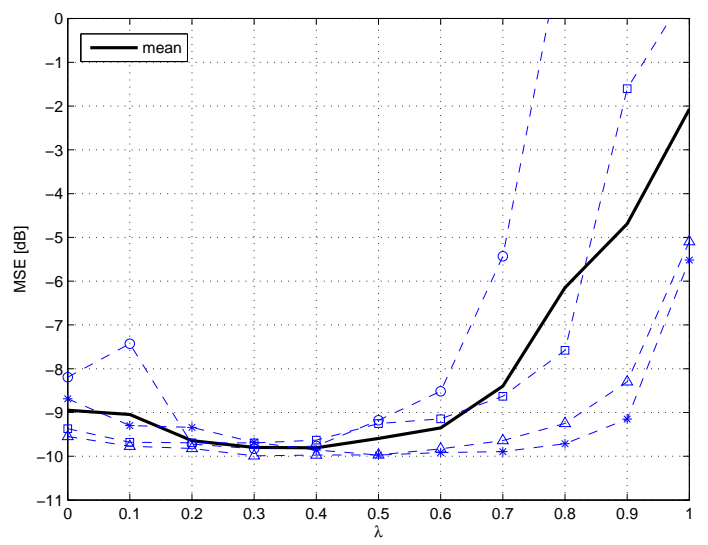

Figure 4.19: MSE vs adaptation factor $\lambda$.

\section{Refining the symbol decisions}

At this point one can repeat data detection using the updated estimates. However, this step may not be necessary, as the entire system operation is contingent upon the assumption that the 
channel varies slowly enough that the gain/phase prediction is accurate.

\section{Predictions for the next block}

Finally, predictions are made for the next block. The gain is predicted simply as

$$
\tilde{\mathbf{A}}^{t, r}(n+1)=\hat{\mathbf{A}}^{t, r}(n)
$$

while the phase predictions are made under the assumption that the estimated motion will remain constant until the next block

$$
\tilde{\alpha}_{k^{\prime}}^{t}(n+1)=\hat{\alpha}_{k^{\prime}}^{t}(n)+2 \pi f_{k^{\prime}} \hat{a}^{t}(n) T^{\prime}
$$

\section{Initialization}

The phases and the Doppler factors are initially set to zero: $\hat{\alpha}_{k^{\prime}}^{t}(0)=0$ and $\hat{a}^{t}(0)=0$. The algorithm starts by estimating the channel during the block $n=0$, which yields the TF coefficients $\hat{\mathbf{A}}^{t, r}(0)$. Full operation starts at $n=1$ with predictions $\tilde{\mathbf{A}}^{t, r}(1)=\hat{\mathbf{A}}^{t, r}(0)$, and $\tilde{\alpha}_{k^{\prime}}^{t}(1)=0$. 


\section{Chapter 5}

\section{Results}

Performance of the SFBC-OFDM system was tested using synthetic data (simulation) as well as real data collected during the June 2010 Mobile MIMO Acoustic Communications Experiment (MACE'10). The test channel used for simulation was constructed to reflect the experimental conditions, which are described below.

\section{$5.1 \quad$ Experiment description}

The experiment was conducted by the Woods Hole Oceanographic Institution (WHOI) at a location 60 miles south of Martha's Vineyard island (see Fig.5.1). During the experiment, the transmitter was deployed from a vessel moving at $0.5 \mathrm{~m} / \mathrm{s}-2 \mathrm{~m} / \mathrm{s}$ in a repeated circular pattern, towards and away from the receiver as shown in Fig.5.2. The signals were recorded at a fixed vertical array located at coordinates $(0,0)$. The geometry of the experimental channel is shown in Fig.5.3.

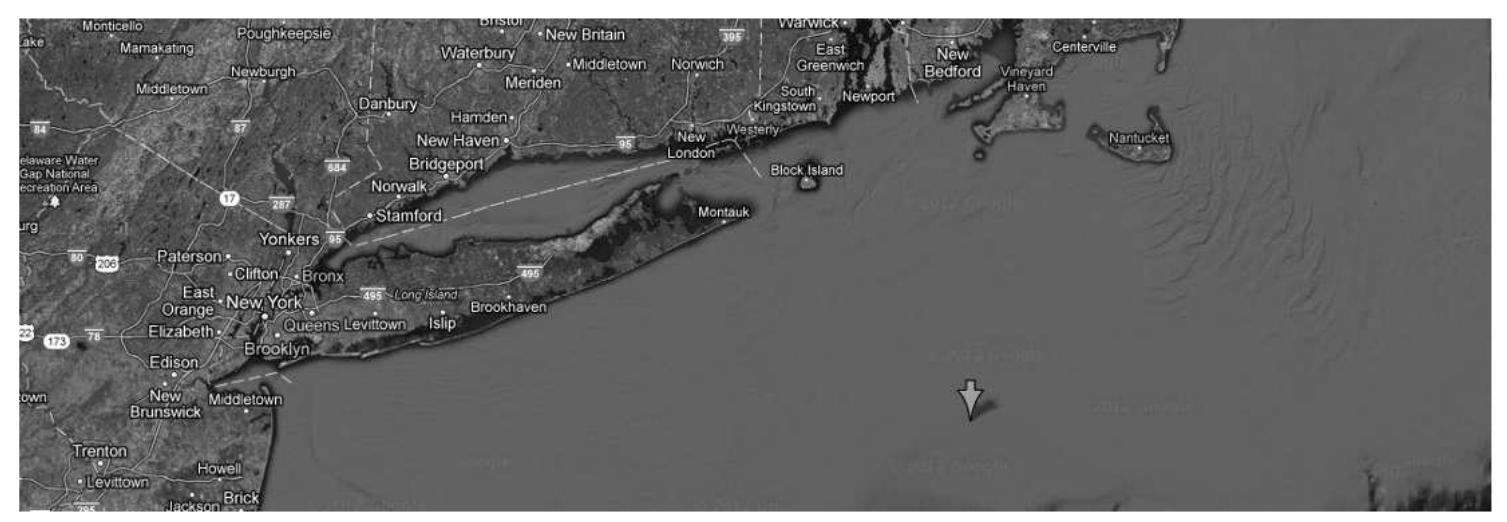

Figure 5.1: Experiment location.

The experiment lasted for seven days, and the Alamouti SFBC signals were transmitted in the $10 \mathrm{kHz}-15 \mathrm{kHz}$ acoustic band in limited intervals during days 5, 6 and 7. Table 5.1 summarizes the signal parameters used in the experiment. QPSK modulation was used on all carriers, whose 


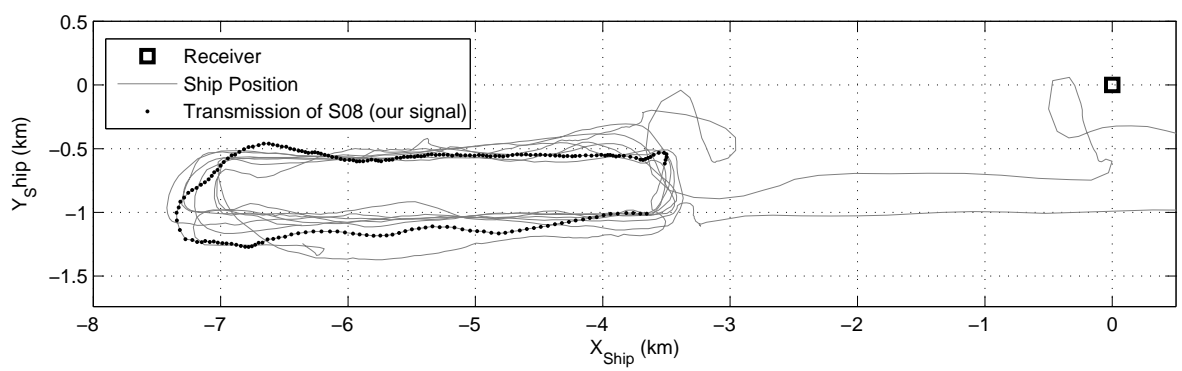

Figure 5.2: Transmitter trajectory.

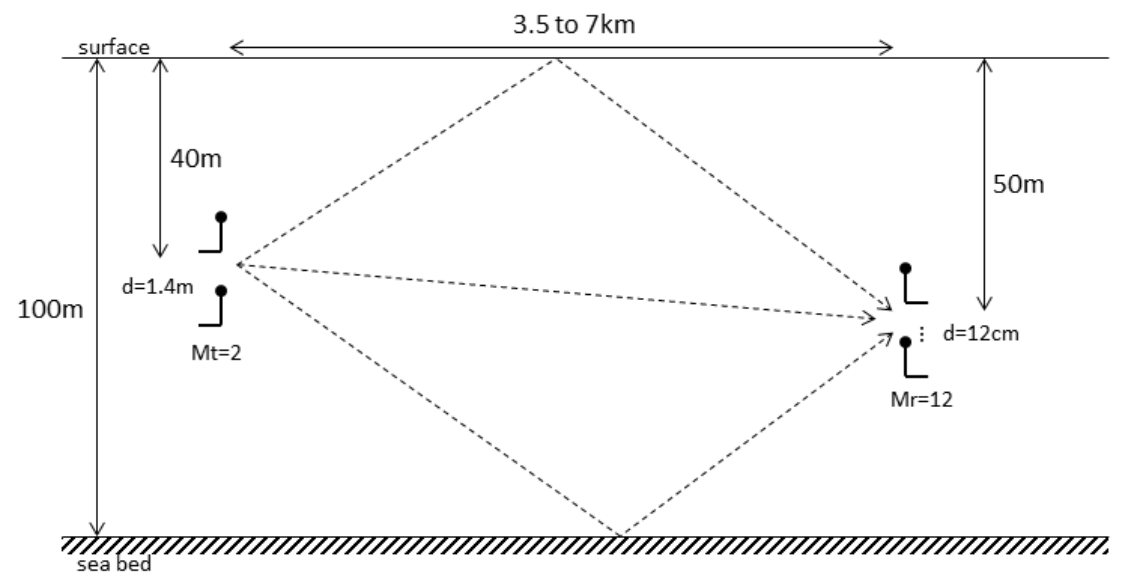

Figure 5.3: Experiment geometry.

number ranged from from 64 to 1024. Transmission was organized in frames, each containing 8192 data symbols divided into a varying number of OFDM blocks. The blocks were separated by a guard interval of $16 \mathrm{~ms}$, and a synchronization probe was inserted at each end of a frame. With adaptive processing, pilot symbols were used only in the first block. The resulting overhead is $0.78 \%$ (with $K=64), 1.56 \%(K=128)$ and $3.13 \%(K=256,512,1024)$. With non-adaptive processing (block-by-block independent detection) the required overhead is $50 \%(K=512)$ and $25 \%(K=1024)$, whereas a $100 \%$ overhead would be needed with $K=256$ or less.

Fig.5.4 shows a snapshot of the channel impulse response (magnitude) obtained directly from the LS estimates. The channel has a sparse structure, and several of the multipath arrivals are well resolved. The total delay spread is about $12 \mathrm{~ms}$ in this case. Throughout the experiment, however, the multipath spread varied between $5 \mathrm{~ms}$ and $16 \mathrm{~ms}$.

\subsection{Simulation results}

The simulation test channel is generated according to the expressions (4.4) and (4.5), where the path gains $h_{p}^{t, r}$ and delays $\tau_{p}^{t, r}(0)$ are initialized using a library of the actual channels from the 
Table 5.1: MACE Experiment signal parameters

\begin{tabular}{|l|l|}
\hline Bandwidth, $B$ & $4883 \mathrm{~Hz}$ \\
\hline First carrier frequency, $f_{0}$ & $10580 \mathrm{~Hz}$ \\
\hline Sampling frequency, $f_{s}$ & $39062 \mathrm{~Hz}$ \\
\hline Number of carriers, $K$ & $64,128,256,512,1024$ \\
\hline Carrier spacing, $\Delta f[\mathrm{~Hz}]$ & $76,38,19,10,5$ \\
\hline OFDM Block duration, $T[\mathrm{~ms}]$ & $13,26,52,104,210$ \\
\hline Guard interval, $T g$ & $16 \mathrm{~ms}$ \\
\hline Adaptation factor, $\lambda$ & 0.4 \\
\hline Symbols per frame, $N_{d}$ & 8192 QPSK \\
\hline Blocks per frame, $N$ & $128,64,32,16,8$ \\
\hline Bitrate, $R$ [kbps] & $4.3,5.9,7.2,8.1,8.7$ \\
\hline Channel code & Hamming $(14,9)$ \\
\hline
\end{tabular}

MACE'10 experiment. Random variation is added to these path gains using a Ricean model, which was found to provide a good match for this type of channel [26]. Specifically, the Rice $\mathcal{K}$ factors are set to $\mathcal{K}_{1}=5$ for the direct path, $\mathcal{K}_{2}=0.5$ for the bottom-reflected path and $\mathcal{K}_{3}=0$ for surface reflections. The random variation follows an AR-1 process with exponentially decaying time-correlation and Doppler spread $B_{d}$.

The arrival time difference (recall the discussion of Sec.4.1.1) is set to $\Delta \tau_{0}^{r}=0.3 \mathrm{~ms}$ for all receiving elements, and the Doppler factors experience a linear increase from 0 at the beginning of a frame to $4 \cdot 10^{-4}$ at the end of a frame.

\subsubsection{System performance}

Fig.5.5 illustrates the bit error rate (BER) as a function of the number of carriers in an adaptive Alamouti SFBC OFDM system. ${ }^{1}$ As a benchmark, we use a single-input multiple-output (SIMO) system implemented with maximal-ratio combining (MRC). The MIMO system performance is also shown in configuration with full channel inversion (4.3), labeled SFBC-X. Each point is a result of averaging over all carriers and 300 frames, each generated using independent noise and fading realizations.

The SFBC system achieves the best performance with 128 and 256 carriers. With more carriers, performance degrades because of the gradual loss of time-coherence and the rise of ICI. With fewer carriers, ( $K=128$ in this example) there is a gradual loss of frequency coherence, which may eventually start to violate the Alamouti assumption (4.13). SFBC-X thus gains a slight advantage at $K=128$. The very poor performance at $K=64$ is an artifact of having insufficiently many pilots to perform channel estimation - at most $K / 2=32$ pilots are available per transmitter, sufficing to cover only $32 / B=6.4 \mathrm{~ms}$ of multipath, while the true multipath spread is about twice as long. (An actual system would not be designed in this manner; the $K=64$ MIMO point is included only for the sake of illustration). The rest of the values represent system configurations in which the

\footnotetext{
${ }^{1}$ Unless stated otherwise, raw (uncoded) BER is shown.
} 

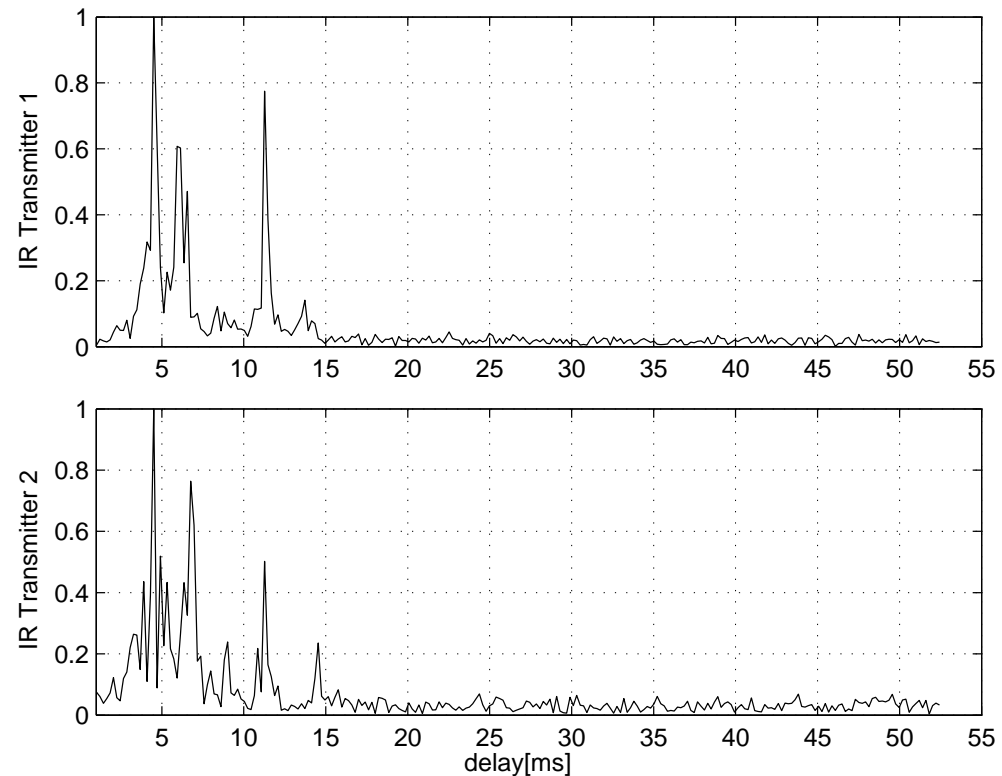

Figure 5.4: Snapshots of channel response observed between the two Alamouti transmitters and a common receiver.

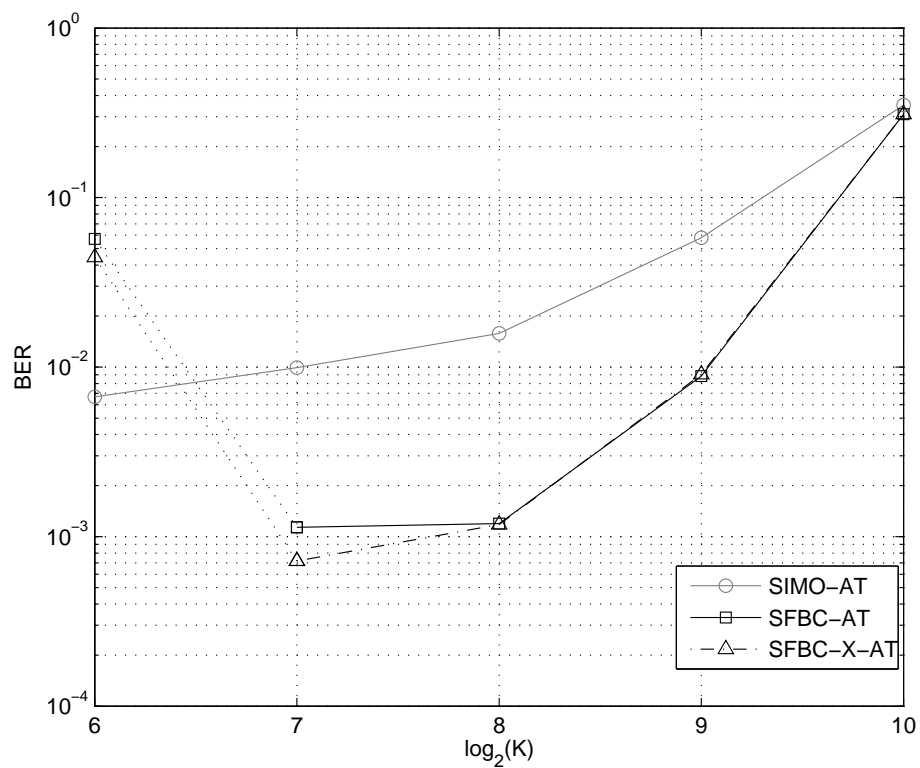

Figure 5.5: Simulation: BER vs. number of carriers. $\mathrm{SNR}=15 \mathrm{~dB}, M_{R}=2$ receiving elements, channel Doppler spread $B_{d}=1 \mathrm{~Hz}$. Label X indicates full channel inversion (4.3).

trade-off between frequency- and time-coherence is well resolved.

In Fig.5.6 we investigate the system performance as a function of the signal-to-noise ratio (SNR), defined as the usual $E_{b} / N_{0}$ figure. STBC refers to the space-time implementation of the 


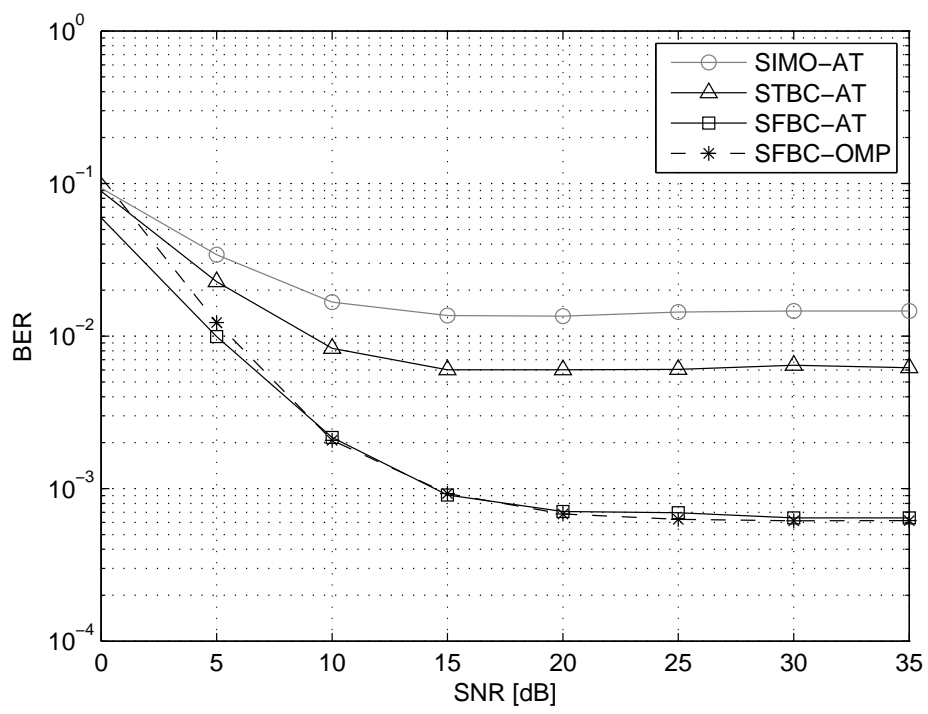

Figure 5.6: Performance comparison between SIMO, STBC and SFBC with different channel estimation algorithms: least-squares with adaptive thresholding (AT) and orthogonal matching pursuit (OMP). $K=256, M_{R}=2$ receivers.

Alamouti code as proposed in [3]. The SFBC system outperforms SIMO and STBC in terms of BER by a factor of 20 and 9, respectively. Labeled as AT is the system that uses LS with adaptive thresholding for channel estimation as described in Sec. 4.4.1, which is compared with channel estimation based on OMP. We note that the two algorithms have almost identical performance. LS-AT offers lower computational complexity, and may thus be preferred. The performance and computational cost of various algorithms will be discussed in more detail in Sec. 5.3.4.

\subsubsection{Effect of desynchronization}

In Fig.5.7 we investigate the effect of synchronization mismatch, i.e. receiver's sensitivity to the difference in the times of signal arrival from the two transmitters. The figure shows the mean squared error (MSE) vs. the delay difference, which is taken to be equal for all the receiving elements, $\Delta \tau_{0}^{r}=\Delta \tau_{0}$. As we conjectured in Sec.4.1.1, the system can tolerate delay differences that do not produce significant TF phase rotation across carriers, and the result of Fig.5.7 testifies to the fact that the performance remains unaltered for delays up to a millisecond. The difference in delay of $1 \mathrm{~ms}$ corresponds to the travel length difference of $1.5 \mathrm{~m}$, which accidentally almost coincides with the transmit element spacing used in the MACE'10 experiment. This distance in turn corresponds to ten wavelengths $\lambda_{0}=c / f_{0}=0.15 \mathrm{~m}$, a separation that is sufficiently large to achieve spatial diversity. 


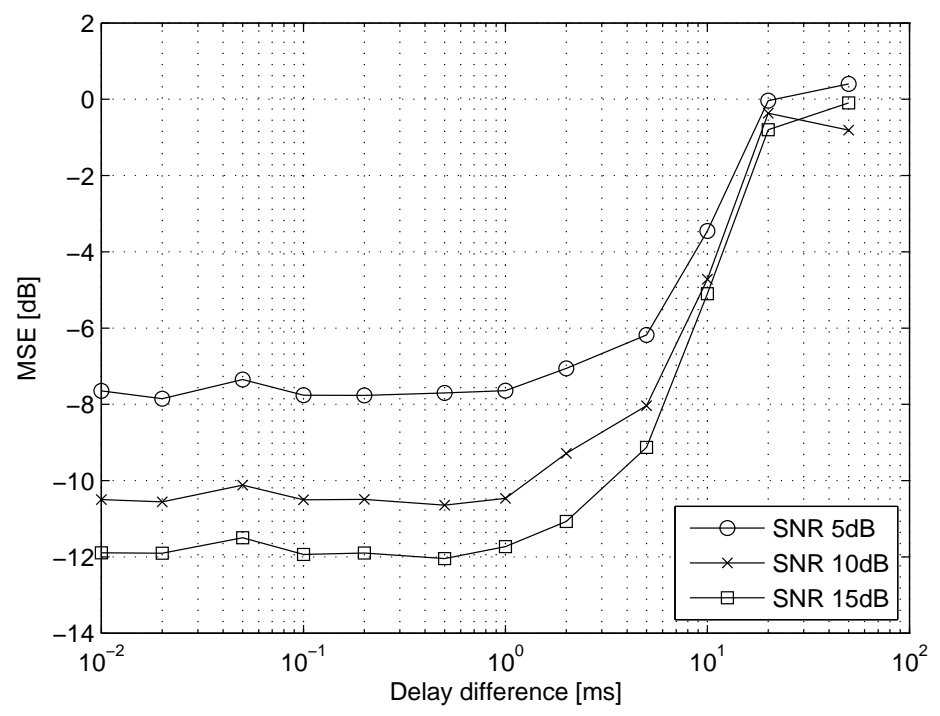

Figure 5.7: Performance sensitivity to synchronization mismatch between transmitters: MSE vs. delay difference $\Delta \tau_{0}(0) . K=256$ carriers, $M_{R}=6$ receiving elements.

\subsubsection{Effect of increased channel variation}

System performance in different channel dynamics, i.e. at different values of the Doppler spread $B_{d}$, is illustrated in Fig.5.8. The gain achieved with SFBC is approximately constant with respect to the SIMO case, provided that both perform channel estimation every block. However, the STBC system requires longer channel coherence time and this fact translates to a limited gain and earlier saturation.

\subsubsection{Effect of Doppler distortion}

Finally, in Fig.5.9 we investigate the system performance as a function of the residual Doppler factor. This result clearly demonstrates the advantages of SFBC over STBC on a time-varying channel. While coding in time requires the channel to remain constant over two adjacent blocks, coding in frequency requires it to stay constant only over one block. As a result, SFBC tolerates higher residual Doppler scales than does STBC (the break-away point at which the BER rapidly increases occurs later for SFBC). A second type of advantage is also evident: as residual Doppler scaling vanished, SFBC mantains better performance. This behaviour is attributed to better handling of the inherent channel variation present in the Ricean-distributed path gains (described in Fig.5.8).

\subsection{Experimental results}

Experimental data available for our study included 87 transmissions performed once every 4 minutes. Each transmission included one frame of OFDM blocks with 64 carriers, one frame with 


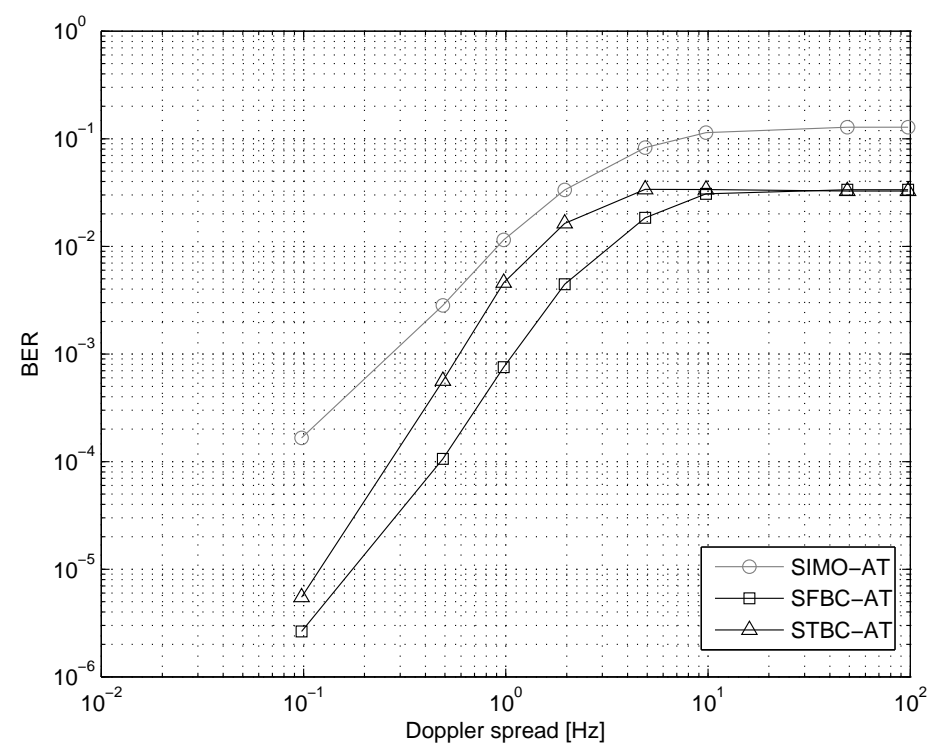

Figure 5.8: Performance comparison between SIMO, STBC and SFBC for different channel variation rates. $\mathrm{SNR}=20 \mathrm{~dB}, K=256, M_{R}=2$ receivers.

128 carriers, etc. During the time when these signals were transmitted, the source moved at a varying velocity, ranging from 0.5 to $2 \mathrm{~m} / \mathrm{s}$. The results of real data processing are presented in terms of BER and MSE averaged over all the blocks and all the carriers, similarly as with the simulation. ${ }^{2}$ The LS-AT algorithm was used for channel estimation in the experimental results.

\subsubsection{System performance}

Fig.5.10 shows the BER as a function of the number of carriers. We observe a similar trend as with synthetic data (Fig. 5.5), with the best performance at $K=256$, corresponding to the carrier spacing $\Delta f=19 \mathrm{~Hz}$. SFBC and SIMO are compared fairly, as the same transmit power was used for both types of signals. Shown also is the method that uses full matrix inversion for data detection (SFBC-X), demonstrating that simple Alamouti detection incurs only a small penalty when the number of carriers is below the optimum. The Alamouti assumption is better justified with more carriers, while the bandwidth efficiency is simultaneously increased. The MSE gain with respect to the SIMO case remains approximately constant for $K \geq 256$, on the order of $2 \mathrm{~dB}$. At $K=64$ and $K=128$, there is a gradual loss of frequency coherence, and a sufficient number of observations is not provided to cover the multipath spread in all situations.

Fig.5.11 shows the MSE evolution in time observed during several hours of one day of the experiment. SFBC outperforms SIMO-MRC uniformly, by about $2 \mathrm{~dB}$ over the 51 consecutive frames. SFBC-ECC refers to the case in which error correction coding is exploited by the receiver to improve the reliability of decisions used for adaptive channel estimation. Coding effectively

\footnotetext{
${ }^{2}$ Those frames in which front-end synchronization failed were not included in statistics.
} 


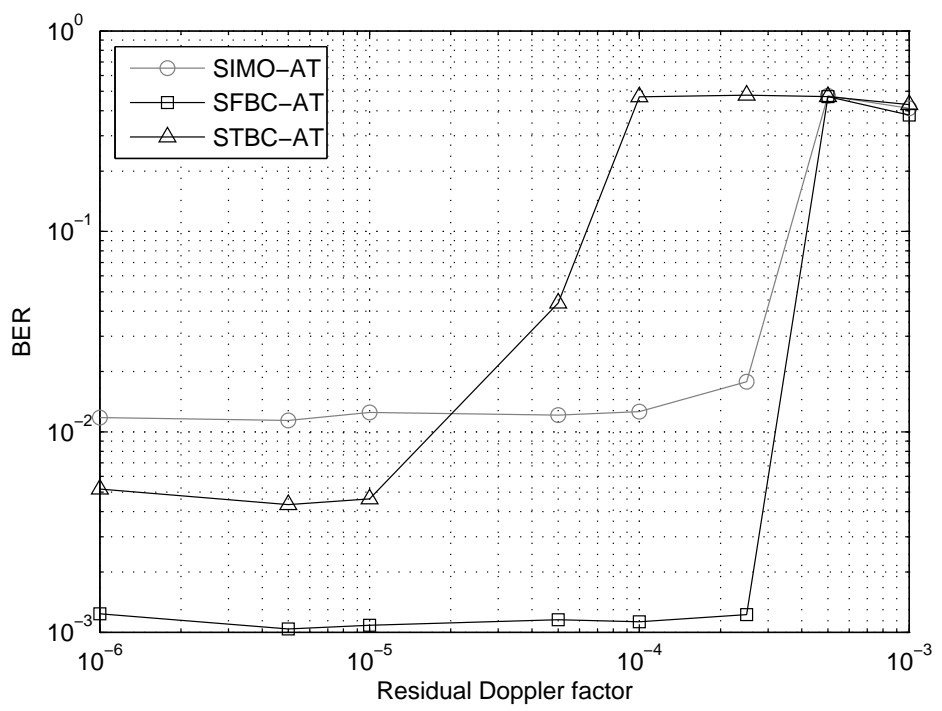

Figure 5.9: Performance comparison between SIMO, STBC and SFBC for different residual relative velocities. $\mathrm{SNR}=15 \mathrm{~dB}, K=256, M_{R}=2$ receivers, $B_{d}=1 \mathrm{~Hz}$.

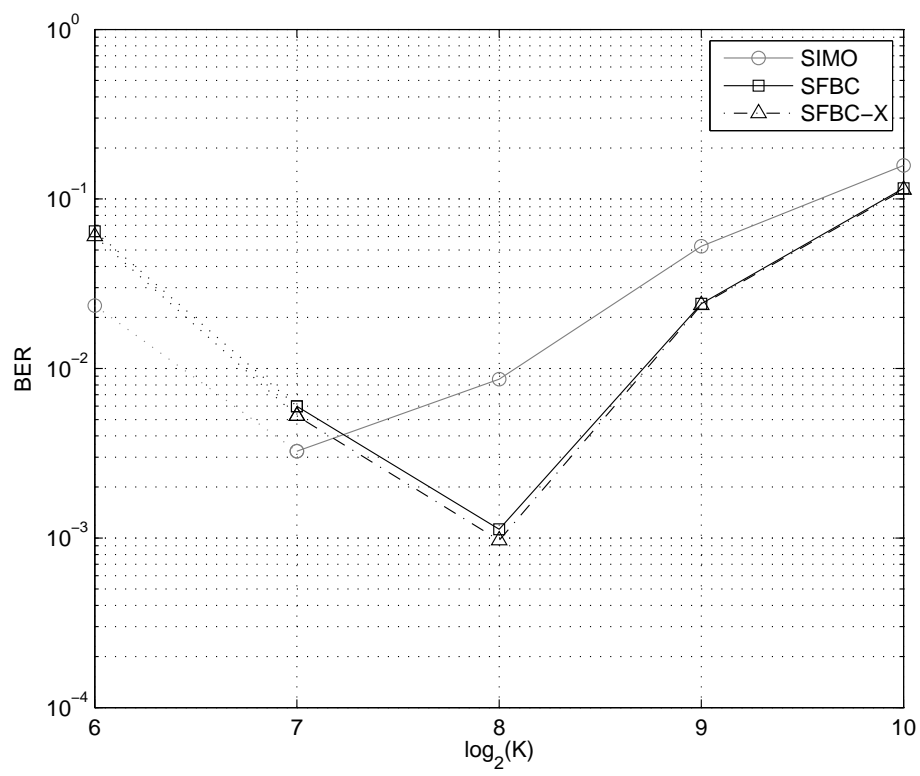

Figure 5.10: Experiment: BER (uncoded) vs. the number of carriers. $M_{R}=12$ receiving elements. Each point represents an average over all carriers and frames.

reduces the MSE below $-7 \mathrm{~dB}$ throughout all the blocks. Comparing the MSE performance to the wind speed reveals an interesting correlation. The MSE is higher during the first three hours while the wind is stronger, and decreases at the end as the wind slows down. The MSE also behaves less erratically during the calmer wind period. Incidentally, this last period is accompanied by an increased transmitter velocity, which does not affect the performance. The largest excursions of 
the MSE are observed at hours 5 and 6.5 when the wind speed reaches highest values. Increased surface activity during those periods is believed to cause faster fading on the scattered paths, causing loss in performance of signal processing.

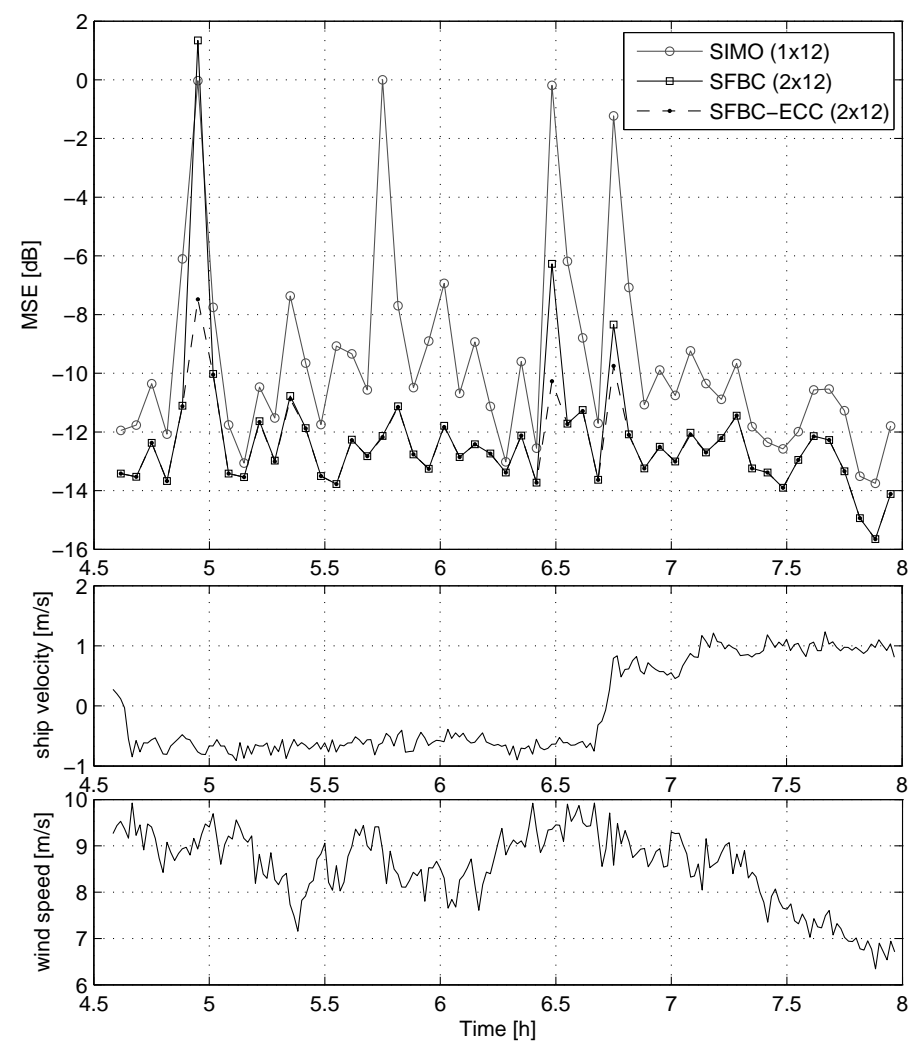

Figure 5.11: MACE experiment, day 5: MSE evolution in time. $K=256, M_{R}=12$ receiving elements.

\subsubsection{Effect of desynchronization}

Fig.5.12 shows the sensitivity to synchronization mismatch. For this measurement, signals from different transmitters were staggered in time, so that they could be synchronized separately, and combined after adding an artificial delay. Similarly as with synthetic data (Fig.5.7), we observe that the performance remains unaffected for delay differences up to about $1 \mathrm{~ms}$. While the delay difference in the current system geometry with co-located transmitters is within this limit, we note that additional synchronization techniques become necessary for cooperative transmission scenarios with spatially distributed transmitters.

\subsubsection{The $\Delta f / 2$ correction}

In Fig.5.13 we investigate the benefits of additional processing applied to the TF coefficient estimates to correct for the $\Delta f / 2$ offset (Sec.4.4.1). This result shows that the $\Delta f / 2$ correction provides a gain when the number of carriers is below the optimum, i.e. when there is a loss of 


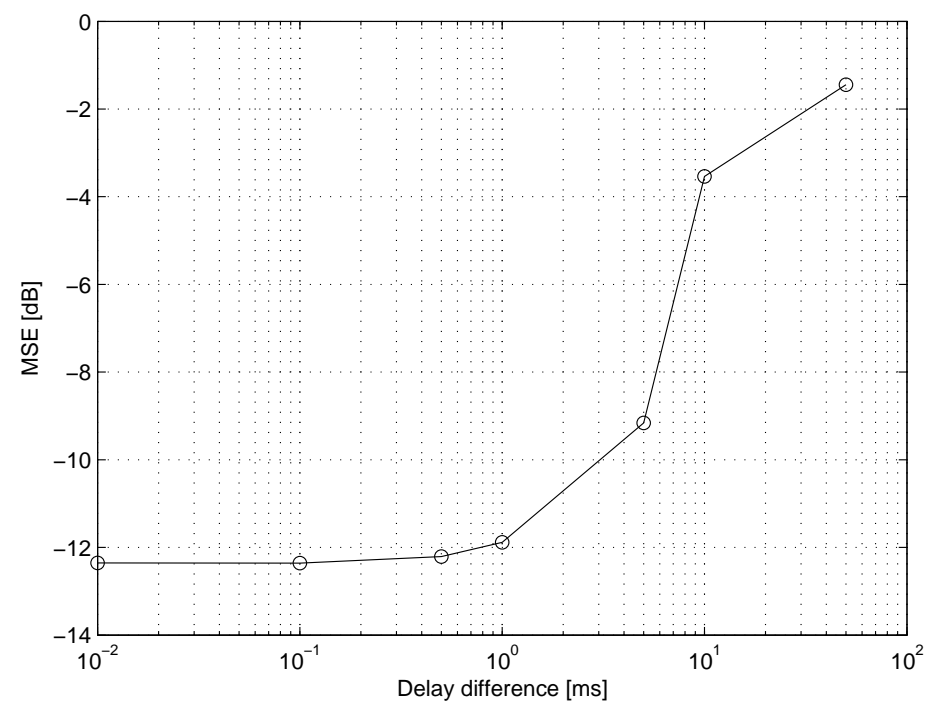

Figure 5.12: Performance sensitivity to synchronization mismatch between transmitters: MSE vs. delay difference $\Delta \tau_{0}(0)$. MACE'10 data with $K=256$ carriers, $M_{R}=12$ receiving elements.

frequency coherence due to the increased carrier separation. The gain is about $2 \mathrm{~dB}$ for $K=64$ and $128 ; 0.5 \mathrm{~dB}$ for $K=256$, and negligible thereafter. The $\Delta f / 2$ correction requires processing with $2 K$-resolution during two steps, hence its gain comes at the price of increased computational complexity.

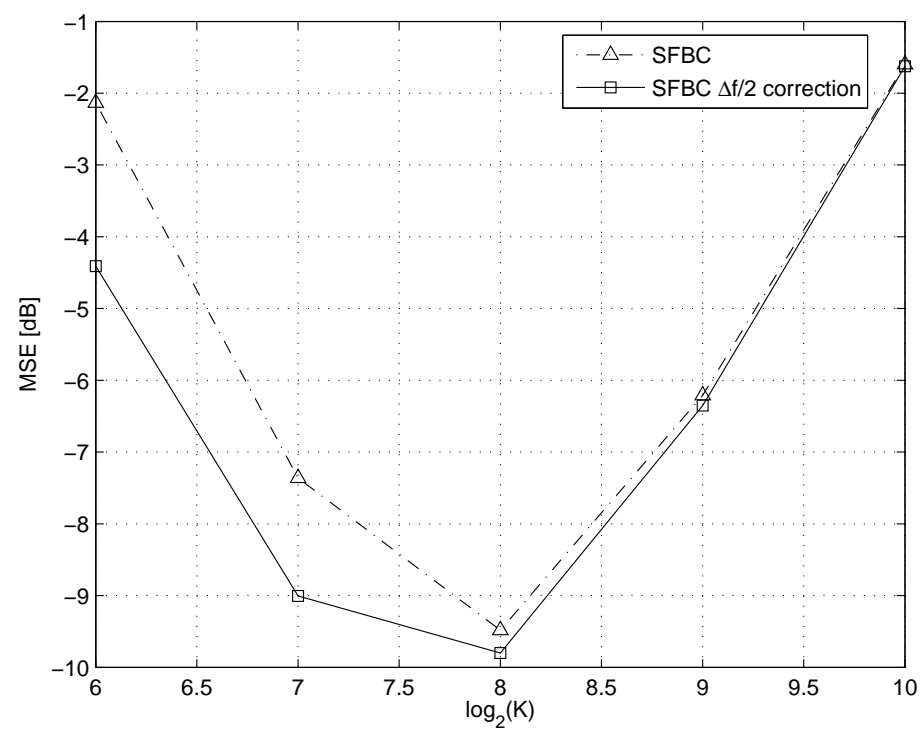

Figure 5.13: System performance with and without the $\Delta f / 2$ correction. Results are shown for a single MACE'10 frame. $M_{R}=12$ receiving elements. 


\subsubsection{Comparison of sparse channel estimation methods}

Finally, we take a closer look at the performance of several channel estimation algorithms, namely LS-AT, LS with a fixed truncation threshold $\gamma$ and OMP. Fig.5.14 shows the performance of LSAT and LS with a fixed threshold. Clearly, adaptive thresholding outperforms fixed thresholding, and in fact represents a bound on its performance. The optimal threshold for a given physical channel depends on the number of carriers. Specifically, it decreases with $K$, as more observations are available for the channel estimator, and, hence, the quality of the estimate improves vis-à-vis noise.

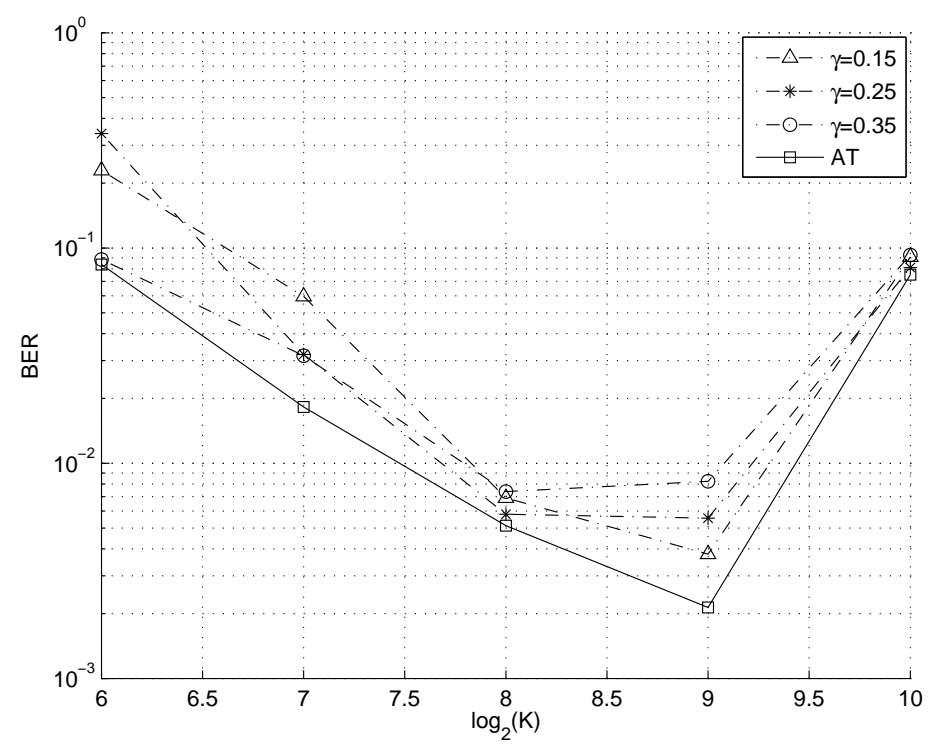

Figure 5.14: Comparison between adaptive-threshold (20 steps) and fixed-threshold methods; single MACE'10 frame. $M_{R}=12$ receiving elements.

To illustrate the performance of adaptive thresholding, we show in Fig. 5.15 several thresholds found by LS-AT, where each curve represents the evolution of the threshold used to estimate each transmitter-receiver channel within an entire frame (32 OFDM blocks for $K=256$ ). Most threshold levels lie in the region between 0.15 and 0.30 but they may change as much as 0.30 from one OFDM block to another. This observation speaks strongly in favor of adaptive threshold setting.

Fig. 5.16 shows the comparison between LS-AT, the OMP algorithm and the ICI-ignorant algorithm proposed in [21]. The latter derives the channel directly from the received signal using a dictionary, which is generated with the transmitted pilots, and has a small loss in performance mainly because it treats the transmitted data as independent. The OMP algorithm solves the model $\check{\mathbf{H}}^{t, r}(n)=\mathbf{F}_{L} \overline{\mathbf{h}}^{t, r}(n)$ using a stopping criterion that measures the relative energy contribution of the last tap obtained. When this energy exceeds a pre-defined threshold (specified in $\mathrm{dB}$ relative to the total energy) the algorithm stops and the last tap is discarded [22]. This criterion 

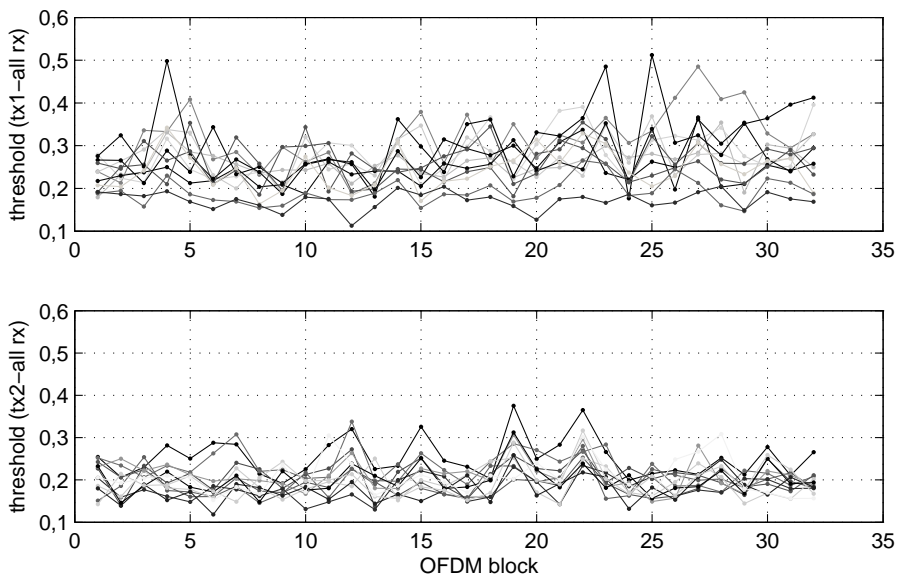

Figure 5.15: Adaptive threshold values for different tx/rx pairs during transmission of one MACE'10 frame. $M_{R}=12$ receiving elements, $K=256$ carriers.

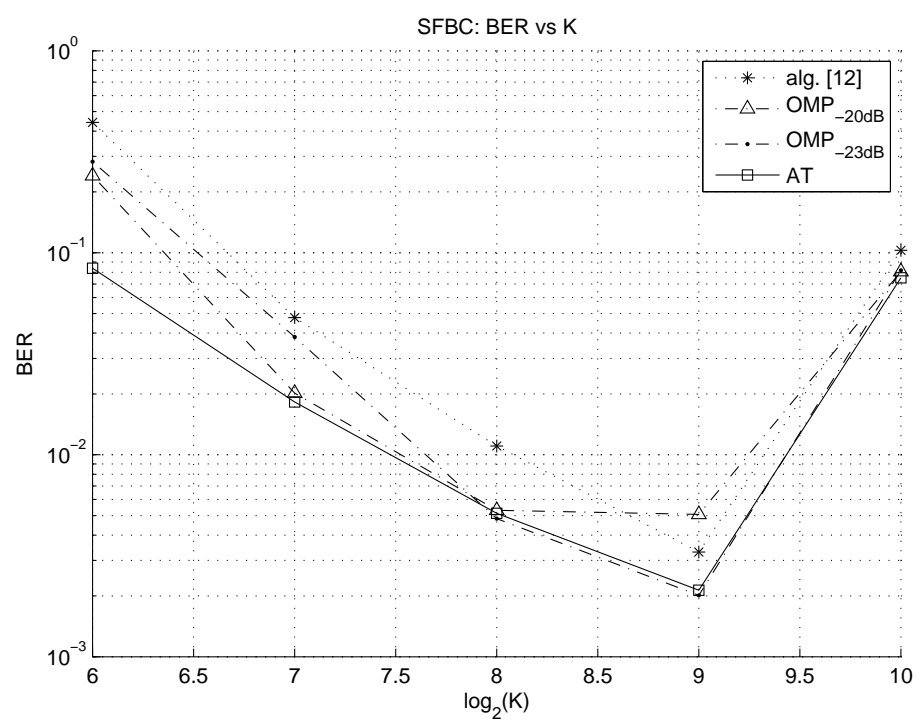

Figure 5.16: Comparison between adaptive-threshold (20 steps), OMP and Algorithm [21]; single MACE'10 frame. $M_{R}=12$ receiving elements.

provides certain adaptability to the channel; however, the threshold has to be defined in terms of the expected noise and multipath intensity profile. As a result, OMP achieves the performance of LS-AT only in certain regions of $K$ (different for each threshold). Fig.5.17 shows an example of channel responses estimated by LS-AT and OMP algorithms.

The computational costs of fixed thresholding, adaptive thresholding and OMP are compared in Table 5.2. The table lists the number (or range) of operations, the average and the maximum number of iterations required to estimate each IR. Each estimated IR of length $K=256$ required an average of $2.2 \cdot 10^{6}$ operations for the OMP algorithm, while LS-AT executed $8 \cdot 10^{4}$ operations, 
Table 5.2: Computational complexity of sparse channel estimation algorithms for an OFDM system with $K$ carriers

\begin{tabular}{r|ccc} 
& LS fixed thr. & LS-AT $(\mathcal{S}=20$ steps $)$ & OMP $(-23 \mathrm{~dB})$ \\
\hline number of operations for & & & \\
initialization & $K^{2}+2 K$ & $K^{2}+K$ & $2 K^{2}+K$ \\
$i$-th iteration & - & $3 K$ & $i^{2}+2 i K+K$ \\
\hline average (max) number of iterations & & & \\
$\mathrm{K}=256$ & - & $20.96(31)$ & $84.45(119)$ \\
$\mathrm{K}=512$ & - & $20.89(29)$ & $68.20(111)$ \\
\hline
\end{tabular}
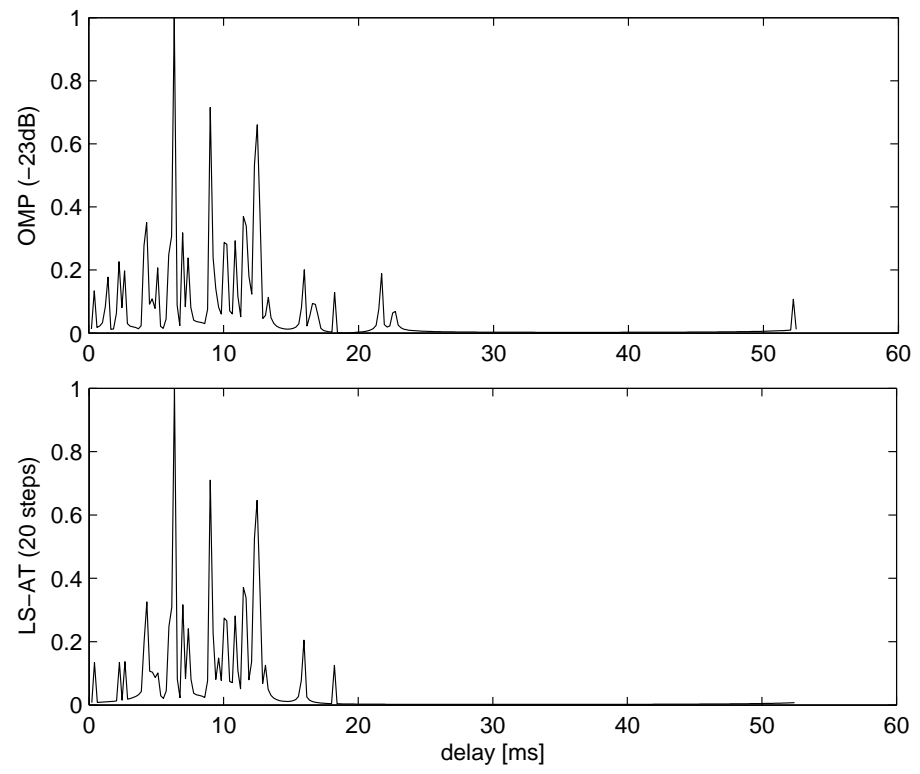

Figure 5.17: Example of channel responses (magnitude) estimated by the OMP and the LS-AT algorithms.

i.e. it was 20 to 30 times faster while offering a comparable performance. The LS-AT algorithm effectively reduces the number of iterations by virtue of its convergence in the time domain, whereas the OMP algorithm requires an iteration for each estimated tap. Since it only requires a $K$ size comparison and less-than- $3 K$ size subtraction per iteration, LS-AT is well conducive to a DSP implementation. 


\section{Chapter 6}

\section{Conclusions and future work}

MIMO spatial diversity was investigated for underwater acoustic communications through the use of Alamouti space-frequency coding coupled with OFDM. The use of space-frequency coding, as opposed to space-time coding, is motivated by the fact that frequency coherence naturally exists between the carriers of a properly designed (ICI-free) OFDM system. While it is needed to support FFT-based OFDM channel equalization, frequency coherence simultaneously supports Alamouti detection, which accomplishes MIMO cross-talk elimination without the need for matrix inversion.

Space-frequency coded OFDM can be used both in a non-adaptive framework where the receiver detects each block of $K$ carriers independently, or in an adaptive framework where the receiver exploits the knowledge of a physical propagation model to track those channel parameters that are varying slowly in time. We proposed a sparse channel identification algorithm based on least squares with adaptive thresholding (LS-AT), and found that this algorithm operates close to orthogonal matching pursuit (OMP), at a lower computational complexity. For the adaptive setting, we proposed an algorithm that targets (i) the Doppler scaling factors corresponding to the two transmitters of the Alamouti pair, and (ii) the respective channel gains that remain slowly-varying once the Doppler shifts have been removed. More specifically, adaptive channel estimation targets the slowly-varying, sparse impulse-response coefficients, and employs further time-smoothing across the OFDM blocks. Channel tracking is enabled by block-adaptive phase correction, which relies on estimating the Doppler scaling factors to predict each carrier's phase for the next OFDM block.

System performance was illustrated through simulation and with real data recorded in a mo-

bile acoustic channel. Experimental results demonstrate the feasibility of space-frequency coded OFDM, with a uniform $2 \mathrm{~dB}$ gain over the SIMO benchmark. The gain is contingent upon sufficient frequency coherence, which is notably present in bandwidth-efficient configurations (256 or 512 carriers in the $5 \mathrm{kHz}$ experimental bandwidth). Using fewer carriers which are more widely spaced causes a loss in frequency coherence (there is also an attendant loss in bandwidth efficiency), while using more carriers causes a loss in time coherence (ICI). Sensitivity to synchronization mismatch between the two transmitters, i.e. the delay difference in the time of their signal arrivals, 
was also investigated. The system was shown to tolerate delay differences typical of co-located transmitters (applications to cooperative MIMO scenarios with spatially separated transmitters would require scheduling). Interesting observations were also made by correlating the observed system performance to the environmental data, and in particular the wind speed. Future work will target the use of differentially coherent detection in the Alamouti MIMO framework. 


\section{Appendix A}

\section{Mathematical proofs}

\section{A.1 Trace inequalities}

Proof. Trace inequalities:

$$
\left.\begin{array}{l}
\operatorname{tr}(\mathbf{A B}) \geq \lambda_{\min }(\mathbf{A}) \operatorname{tr}(\mathbf{B}) \\
\operatorname{tr}(\mathbf{A B}) \leq \lambda_{\max }(\mathbf{A}) \operatorname{tr}(\mathbf{B})
\end{array}\right\} \mathbf{A}, \mathbf{B} \text { hermitian positive-definite }
$$

Let us consider an arbitrary hermitian positive-definite matrix ${ }^{1} \mathbf{A} \in \mathbb{C}^{m \times m}$ and its eigendecomposition

$$
\mathbf{A}=\mathbf{Q} \mathbf{\Lambda} \mathbf{Q}^{-1}, \quad \mathbf{Q Q}^{H}=\mathbf{I}_{m}
$$

where $\boldsymbol{\Lambda}$ is a diagonal matrix containing the non-negative real eigenvaules sorted by magnitude, i.e. $\lambda_{A 1} \geq \lambda_{A 2} \geq \ldots \geq 0$

$$
\boldsymbol{\Lambda}=\left[\begin{array}{lll}
\lambda_{A 1} & & \\
& \ddots & \\
& & \lambda_{A m}
\end{array}\right]
$$

Also consider another arbitrary hermitian positive-definite matrix $\mathbf{B} \in \mathbb{C}^{m \times m}$ and its decomposition in A's eigenspace

$$
\mathbf{B}=\mathbf{Q P Q}^{-1}
$$

where $\mathbf{P}$ is not necessarily a diagonal matrix, except if $\mathbf{B} \propto \mathbf{A}$,

$$
\mathbf{P}=\left[\begin{array}{ccc}
p_{11} & \ldots & p_{1 m} \\
\vdots & \ddots & \vdots \\
p_{m 1} & \ldots & p_{m m}
\end{array}\right]
$$

\footnotetext{
${ }^{1}$ Note that in the above uses of this property, matrices are hermitian positive-definite because are built with a structure $\mathbf{C}=\mathbf{D D}^{H}$
} 
This matrix verifies the following

$$
\operatorname{tr}(\mathbf{B})=\operatorname{tr}\left(\mathbf{Q}^{-1} \mathbf{Q P}\right)=\operatorname{tr}(\mathbf{P})
$$

hence,

$$
\sum_{i=1}^{m} p_{i i}=\sum_{i=1}^{m} \lambda_{B i}
$$

Since $\mathbf{B}$ is a positive-definite matrix, it verifies

$$
\mathbf{z}^{H} \mathbf{B} \mathbf{z}>0 \quad \mathbf{z} \neq \mathbf{0} \quad \mathbf{z} \in \mathbb{R}^{m}
$$

and this implies that all matrices $\mathbf{P}$ generated with $\mathbf{P}=\mathbf{Z}^{H} \mathbf{B Z}$ (where $\mathbf{Z}$ is any real matrix with non-zero rows) verify $p_{i i} \geq 0$, so all elements in the diagonal of $\mathbf{P}$ are positive

$$
p_{i i} \geq 0
$$

The trace of $\mathbf{A B}$ product results

$$
\operatorname{tr}(\mathbf{A B})=\operatorname{tr}\left(\mathbf{Q} \mathbf{\Lambda} \mathbf{Q}^{-1} \mathbf{Q} \mathbf{P} \mathbf{Q}^{-1}\right)=\operatorname{tr}(\mathbf{\Lambda} \mathbf{P})=\sum_{i=1}^{m} \lambda_{A i} p_{i i}
$$

isolating $p_{11}$ from (A.6) and substituting into (A.9) we have

$$
\operatorname{tr}(\mathbf{A B})=\lambda_{A 1}\left(\sum_{i=1}^{m} \lambda_{B i}-\sum_{j=2}^{m} p_{j j}\right)+\sum_{i=2}^{m} p_{i i} \lambda_{A i}=\lambda_{A 1} \sum_{i=1}^{m} \lambda_{B i}+\sum_{j=2}^{m} p_{j j}\left(\lambda_{A i}-\lambda_{A 1}\right)
$$

Bearing in mind from (A.8) that $p_{j j} \geq 0$ and $\lambda_{A 1}=\lambda_{\max }(\mathbf{A})$, we have

$$
\sum_{j=2}^{m} p_{j j}\left(\lambda_{A i}-\lambda_{A 1}\right) \leq 0
$$

and consequently

$$
\operatorname{tr}(\mathbf{A B})=\lambda_{\max }(\mathbf{A}) \operatorname{tr}(\mathbf{B})+\sum_{j=2}^{m} p_{j j}\left(\lambda_{A i}-\lambda_{A 1}\right) \leq \lambda_{\max }(\mathbf{A}) \operatorname{tr}(\mathbf{B})
$$

so finally

$$
\operatorname{tr}(\mathbf{A B}) \leq \lambda_{\max }(\mathbf{A}) \operatorname{tr}(\mathbf{B})
$$

The analogous inequality can be found the same way but isolating and substituting $p_{m m}$ in (A.10). 
Proof. Trace inequality:

$$
\operatorname{tr}^{2}\left(\mathbf{C}^{H} \mathbf{D}\right) \leq \operatorname{tr}\left(\mathbf{C} \mathbf{C}^{H}\right) \cdot \operatorname{tr}\left(\mathbf{D} \mathbf{D}^{H}\right)
$$

Starting with the property $\operatorname{tr}(\mathbf{A})=\sum_{i} \lambda_{A i}$ :

$$
\operatorname{tr}\left(\mathbf{C C}^{H}\right)=\sum_{i} \lambda_{C i}^{2} \quad \operatorname{tr}\left(\mathbf{D D}^{H}\right)=\sum_{i} \lambda_{D i}^{2}
$$

and considering the above proven inequality $\operatorname{tr}(\mathbf{A B}) \leq \lambda_{\max }(\mathbf{A}) \operatorname{tr}(\mathbf{B})$ we have

$$
\lambda_{\max }(\mathbf{C})^{2} \operatorname{tr}(\mathbf{D})^{2}=\lambda_{C 1}^{2} \sum_{i} \lambda_{D i}^{2} \leq \sum_{i} \lambda_{C i}^{2} \sum_{i} \lambda_{D i}^{2}=\operatorname{tr}\left(\mathbf{C} \mathbf{C}^{H}\right) \cdot \operatorname{tr}\left(\mathbf{D D}^{H}\right)
$$

and, hence,

$$
\operatorname{tr}^{2}\left(\mathbf{C}^{H} \mathbf{D}\right) \leq \lambda_{\max }(\mathbf{C})^{2} \operatorname{tr}(\mathbf{D})^{2} \leq \operatorname{tr}\left(\mathbf{C} \mathbf{C}^{H}\right) \cdot \operatorname{tr}\left(\mathbf{D} \mathbf{D}^{H}\right)
$$

then the following is directly satisfied

$$
\operatorname{tr}^{2}\left(\mathbf{C D}^{H}\right) \leq \operatorname{tr}\left(\mathbf{C} \mathbf{C}^{H}\right) \cdot \operatorname{tr}\left(\mathbf{D} \mathbf{D}^{H}\right)
$$




\section{Bibliography}

[1] B. Li, J. Huang, S. Zhou, K. Ball, M. Stojanovic, L. Freitag, and P. Willett, "Mimo-ofdm for high-rate underwater acoustic communications," Oceanic Engineering, IEEE Journal of, vol. 34, no. 4, pp. $634-644$, oct. 2009.

[2] M. Stojanovic, "Mimo ofdm over underwater acoustic channels," in Signals, Systems and Computers, 2009 Conference Record of the Forty-Third Asilomar Conference on, nov. 2009, pp. $605-609$.

[3] B. Li and M. Stojanovic, "A simple design for joint channel estimation and data detection in an alamouti ofdm system," in OCEANS 2010, sept. 2010, pp. $1-5$.

[4] S. Alamouti, "A simple transmit diversity technique for wireless communications," Selected Areas in Communications, IEEE Journal on, vol. 16, no. 8, pp. 1451 -1458, oct 1998.

[5] K. Lee and D. Williams, "A space-frequency transmitter diversity technique for ofdm systems," in Global Telecommunications Conference, 2000. GLOBECOM '00. IEEE, vol. 3, 2000, pp. $1473-1477$ vol.3.

[6] D.-B. Lin, P.-H. Chiang, and H.-J. Li, "Performance analysis of two-branch transmit diversity block-coded ofdm systems in time-varying multipath rayleigh-fading channels," Vehicular Technology, IEEE Transactions on, vol. 54, no. 1, pp. 136 - 148, jan. 2005.

[7] B. Li, S. Zhou, M. Stojanovic, L. Freitag, and P. Willett, "Multicarrier communication over underwater acoustic channels with nonuniform doppler shifts," Oceanic Engineering, IEEE Journal of, vol. 33, no. 2, pp. 198 -209, april 2008.

[8] K. Tu, T. Duman, J. Proakis, and M. Stojanovic, "Receiver design for ofdm over dopplerdistorted underwater acoustic channels," Oceanic Engineering, IEEE Journal of, to appear.

[9] W. Li and J. Preisig, "Estimation of rapidly time-varying sparse channels," Oceanic Engineering, IEEE Journal of, vol. 32, no. 4, pp. 927 -939, oct. 2007.

[10] M. Stojanovic, "Underwater acoustic communications: Design considerations on the physical layer," in Wireless on Demand Network Systems and Services, 2008. WONS 2008. Fifth Annual Conference on, jan. 2008, pp. $1-10$. 
[11] _ - "On the relationship between capacity and distance in an underwater acoustic channel," in First ACM International Workshop on Underwater Networks (WUWNet), Sept. 2006.

[12] L. Berkhovskikh and Y. Lysanov, Fundamentals of ocean acoustics. New York: Springer, 1982.

[13] R. Coates, Underwater acoustic systems. Houndmills, Basingstoke, Hampshire: Macmillan, 1990.

[14] J. Cooley and J. Tukey, "An algorithm for the machine calculation of complex fourier series," in Math Comp., vol. 19, 1965, pp. 297-301.

[15] J. Proakis and M. Salehi, Digital communications, 5th ed. McGraw-Hill, 2007.

[16] A. Molisch, Wireless communications. Chichester, England; Hoboken, NJ: John Wiley \& sons, 2005.

[17] D. Tse and P. Viswanath, Fundamentals of wireless communication. Cambridge: Cambridge University Press, 2005.

[18] A. Paulraj, Introduction to space-time wireless communications. Cambridge, New York: Cambridge University Press, 2003.

[19] V. Tarokh, N. Seshadri, and A. Calderbank, "Space-time codes for high data rate wireless communication: performance criterion and code construction," Information Theory, IEEE Transactions on, vol. 44, no. 2, pp. $744-765$, mar 1998.

[20] L. Zheng and D. Tse, "Diversity and multiplexing: a fundamental tradeoff in multiple-antenna channels," Information Theory, IEEE Transactions on, vol. 49, no. 5, pp. 1073 - 1096, may 2003 .

[21] C. Berger, S. Zhou, J. Preisig, and P. Willett, "Sparse channel estimation for multicarrier underwater acoustic communication: From subspace methods to compressed sensing," in OCEANS 2009 - EUROPE, may 2009, pp. 1 -8.

[22] A. Radosevic, T. Duman, J. Proakis, and M. Stojanovic, "Selective decision directed channel estimation for uwa ofdm systems," in Communication, Control, and Computing (Allerton), 2011 49th Annual Allerton Conference on, sept. 2011, pp. 647 -653.

[23] R. Burden and J. Faires, Numerical analysis, 3rd ed. PWS Publishers, 1985.

[24] Y. Kang, K. Kim, and H. Park, "Efficient dft-based channel estimation for ofdm systems on multipath channels," Communications, IET, vol. 1, no. 2, pp. 197 -202, april 2007.

[25] J. Oliver, R. Aravind, and K. Prabhu, "Sparse channel estimation in ofdm systems by threshold-based pruning," Electronics Letters, vol. 44, no. 13, pp. 830 -832, 192008. 
[26] P. Qarabaqi and M. Stojanovic, "Statistical modeling of a shallow water acoustic communication channel," in Underwater Acoustic Measurements Conference, June 2009. 